\title{
DESIGN AND VERIFICATION OF A LABVIEW AUTOMATED ANTENNA RADIATION PATTERN MEASUREMENT SYSTEM
}

\author{
A Thesis \\ Presented to \\ the Faculty of California Polytechnic State University, \\ San Luis Obispo
}

\author{
In Partial Fulfillment \\ of the Requirements for the Degree \\ Master of Science in Electrical Engineering
}

By

Dylan Curtis Bloom

July 2018 
(C) 2018

Dylan Curtis Bloom

ALL RIGHTS RESERVED 
COMMITTEE MEMBERSHIP

TITLE: Design and Verification of a LabVIEW Automated Antenna Radiation Pattern Measurement System

AUTHOR: Dylan Curtis Bloom

DATE SUBMITTED: July 2018

COMMITTEE CHAIR: Dennis Derickson, Ph.D.

Associate Professor of Electrical Engineering

COMMITTEE MEMBER: Tina Smilkstein, Ph.D.

Associate Professor of Electrical Engineering

COMMITTEE MEMBER: Xiaomin Jin, Ph.D.

Associate Professor of Electrical Engineering 


\begin{abstract}
Design and Verification of a LabVIEW Automated Antenna Radiation Pattern

Measurement System

Dylan Curtis Bloom
\end{abstract}

In 2014, Toyon Research Corporation donated a 6'x6'x8' anechoic chamber to the Cal Poly EE department to advance student education in the areas of antennas and wireless communications. An anechoic chamber is a room designed to suppress electromagnetic radiation reflections so that accurate measurement of radio frequency (RF) systems such as wireless radios and antennas can take place. Despite the fact that Cal Poly already has a larger anechoic chamber, primarily used for antenna characterization, it is the purpose of this project to design, code, and equip the new chamber so that it performs at least as good, or better than, the existing chamber.

Radiation pattern is a key characteristic that describes the directionality or gain of an antenna, and it is important for quantifying and qualifying how an antenna will perform as part of a wireless communication system. Radiation pattern measurement can be time consuming because it requires measuring an antenna's transmission or reception in in all directions. In both the old and new antenna measurement systems (AMS), a vector network analyzer (VNA) measures signals transmitted between the antenna under test (AUT) and an RF field probe within the antireflective environment of the anechoic chamber. The new system synchronizes VNA measurement with the rotation of the AUT using the automation software LabVIEW and a Sunol Sciences FS-121 antenna positioner. Then, Matlab plots the data collected by LabVIEW as well as calculates useful antenna metrics including half power beam width (HPBW) and directivity. LabVIEW also makes the AMS easy to operate because of its graphical user interface. 
The new anechoic chamber completes measurements faster than Cal Poly's existing chamber, with good accuracy and ease of use. The new chamber works best with smaller antennas at frequencies up to $6 \mathrm{GHz}$ and beyond. Radiation patterns have good SNR, and match with simulations and measurements done in the larger chamber. However, due to its smaller size the new AMS is not a replacement for the existing larger system when testing antennas larger than $1 \mathrm{~m}$ or at frequencies below $2 \mathrm{GHz}$. 


\section{ACKNOWLEDGMENTS}

I dedicate this thesis to my parents, Brian Bloom and Sara Sutton for having me and raising me with all their love and encouragement. I would also like to thank my stepfather and step-mother, Ted Perle and Debbie Bloom, for believing in me and supporting me ever since they met me.

I want to thank Evan Mckee for his partnership in designing and testing this system and making it become a reality, as well as our advisor Dr. Dean Arakaki for enabling this project and offering his knowledge and support throughout. I also want to acknowledge the work of Aldo Chow for helping with the planning stages of this project.

Finally, I want to thank Dr. Dennis Derickson for his leadership throughout my time here at Cal Poly, and for believing in me when I needed it most. Dr. Tina Smilkstein, for being an inspiration to me and advising me on many other projects along the way. And Dr. Xiaomin Jin for taking a chance on me. 


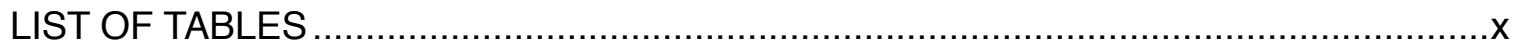

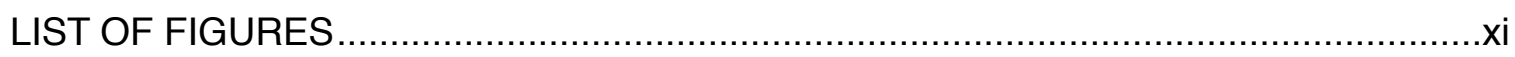

\section{CHAPTER}

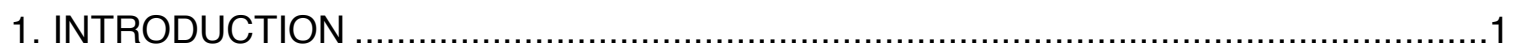

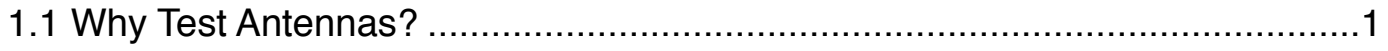

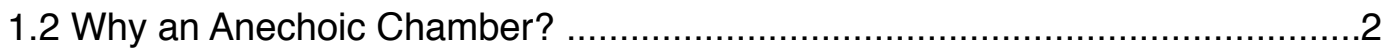

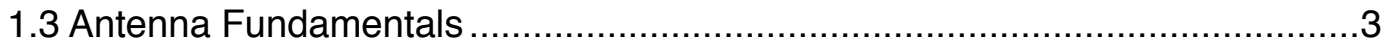

1.3.1 Antenna Coordinate System ......................................................

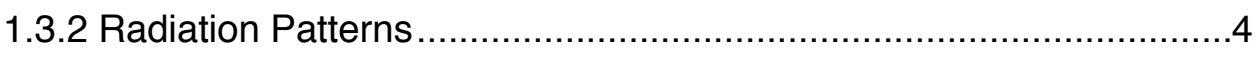

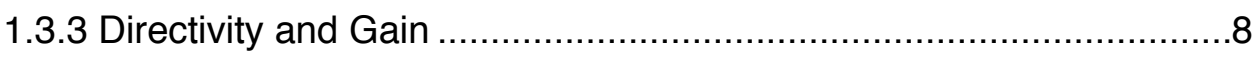

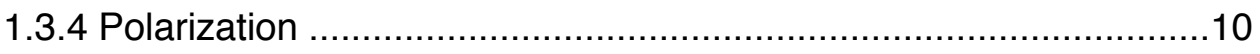

1.3.5 The Near and Far Field ……………………….....................13

2. ANECHOIC CHAMBER AND TEST EQUIPMENT ……........................................15

2.1 The Microwave Lab Anechoic Chamber .....................................................15

2.2 Choosing an AUT Positioner .....................................................................17

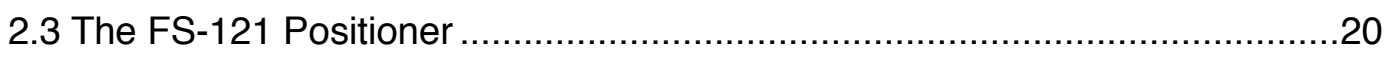

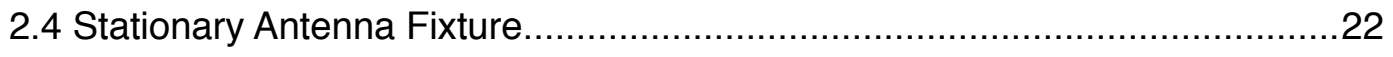

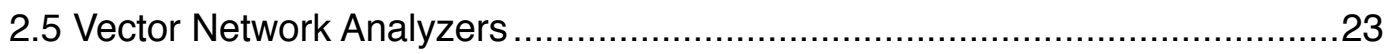

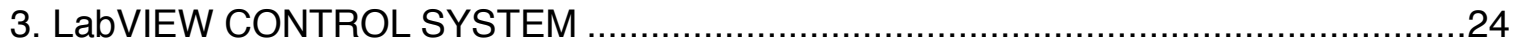

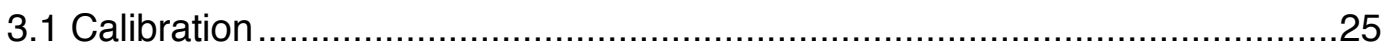

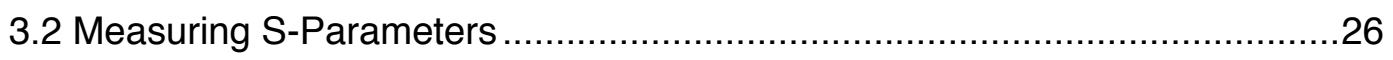

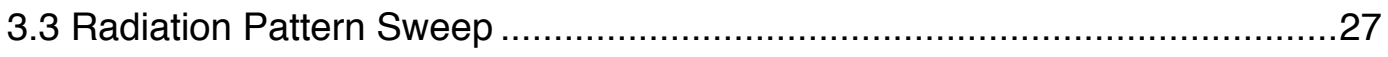




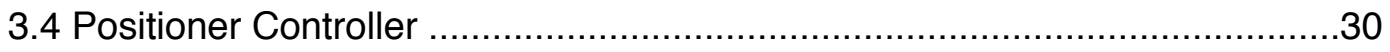

3.4.1 Auto Locate Boresight ...............................................................

3.5 UI Features and Smart Controls ...............................................................

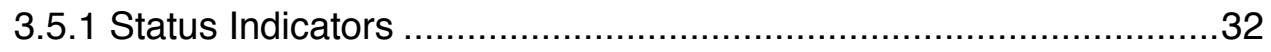

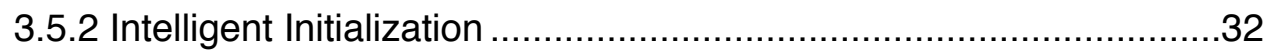

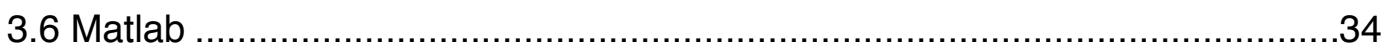

3.6.1 Radiation Pattern Plot ..............................................................

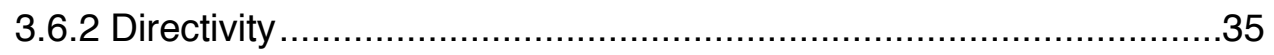

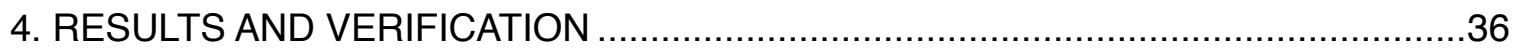

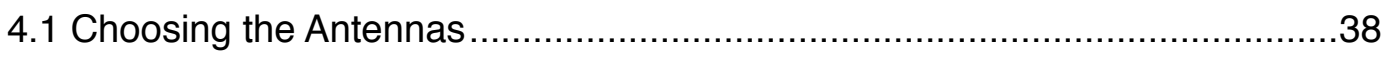

4.2 Mathematical Model for Nodes and Nulls ……..........................................40

4.3 Radiation Patterns Graphed on Polar Plots ...............................................41

4.4 Realizations about Calibration Procedure .................................................4

4.5 New Antenna Patterns with Calibrated Equipment .....................................50

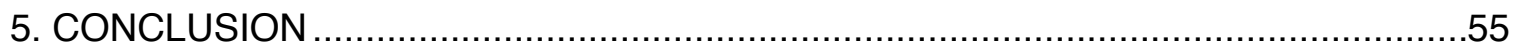

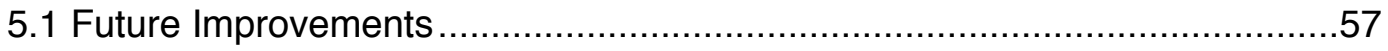

5.1.1 Antenna Separation Distance Tests ………………………….....57

5.1.2 Free Space Material Measurements .............................................57

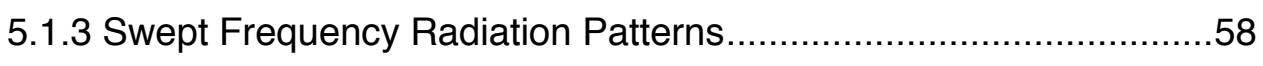

5.1.4 Time Domain Gating …………................................................58

5.1.5 Chamber Calibration for Active Measurements..............................59

6. CREATING THE ANTENNA DESIGN EXPERIMENT ……....................................60

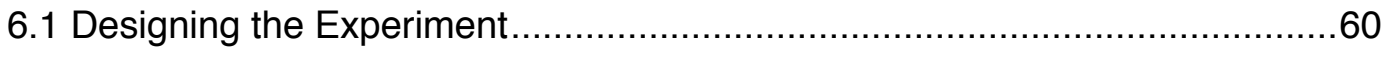

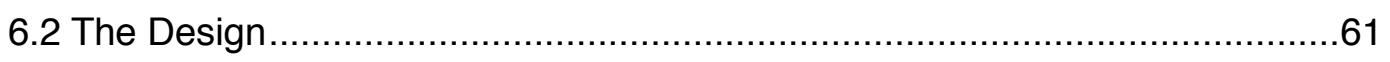

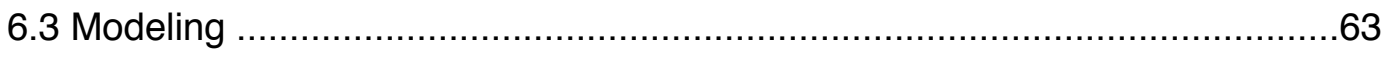




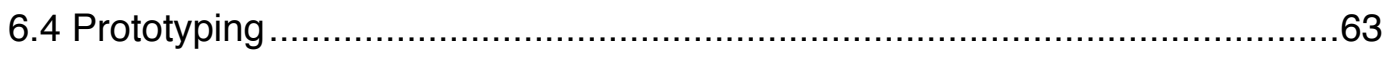

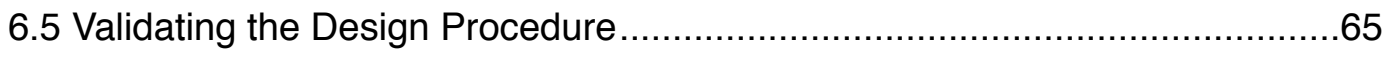

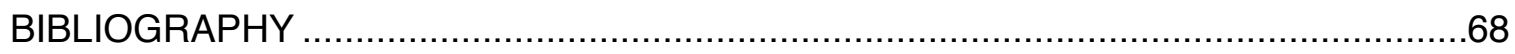

APPENDICES

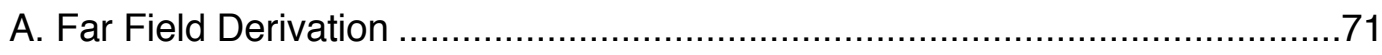

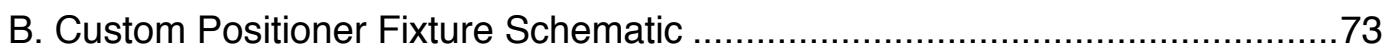

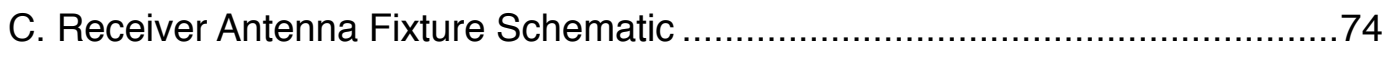

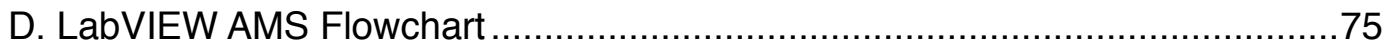

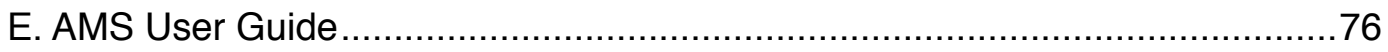

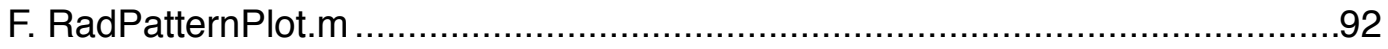

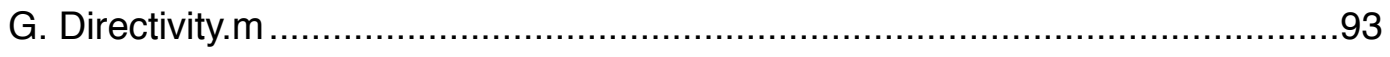

H. HFSS 2.4 GHz Pyramidal Horn Tutorial ..........................................95 


\section{LIST OF TABLES}

Table

1. Potential Antenna Positioners and their Features ............................................19

2. VNA Models, Frequency Ranges, and Availability ...........................................23

3. Differences Between Small and Large Anechoic Chambers ..................................37

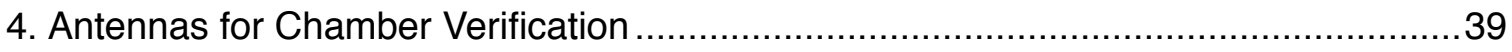

5. Antenna Far Field Distances and Nulls............................................................... 41

6. Original Calibration Procedure for Both Antenna Measurement Systems ..................48

7. New Calibration Procedure for Both Antenna Measurement Systems ......................49

8. Summary of Expected vs Observed Differences and Similarities between

Hangar Lab Chamber and Microwave Lab Chamber ........................................ 56

9. Standard Rectangular Waveguide Dimensions [13] .......................................62 


\section{LIST OF FIGURES}

Figure

Page

1. Pyramidal Horn Antenna in the Cal Poly Microwave Lab Anechoic Chamber ............2

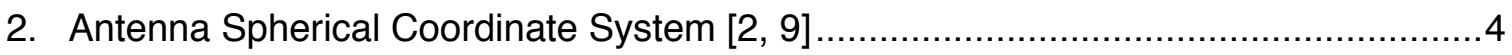

3. Normalized Radiation Field Pattern and its Components [1] ................................

4. (a) Pattern Measurement Conceptualized (b) Model Positioner [2] .........................6

5. Illustration of S-parameters for a Two-Port System ............................................

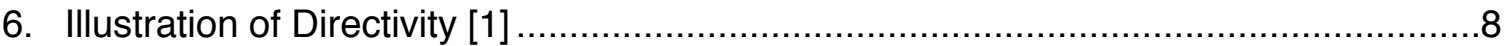

7. Spatial Behavior of Electric (solid) and Magnetic (dashed) Fields of a Linearly (vertical) Polarized Wave for a Fixed Instant of Time [1] ..........................10

8. Some Polarization States (the wave is approaching) [1] .................................11

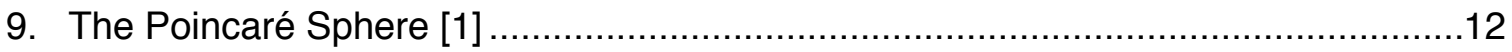

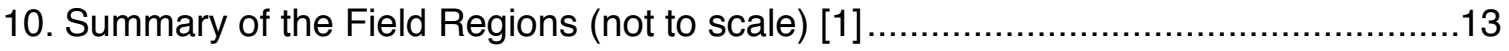

11. Cross Section of the Cal Poly Microwave Lab Anechoic Chamber (to scale)...........16

12. FS-121 Positioner with Custom Mast (left) and Turntable (right) ...........................21

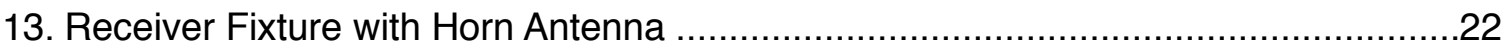

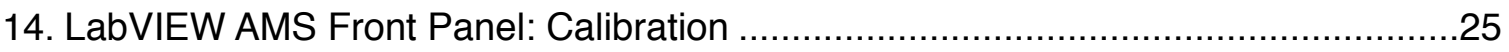

15. LabVIEW AMS Front Panel: S-parameter Measurement......................................26

16. LabVIEW AMS Front Panel: Radiation Pattern (uncalibrated) ...............................27

17. LabVIEW AMS Front Panel: Positioner Controller ................................................30

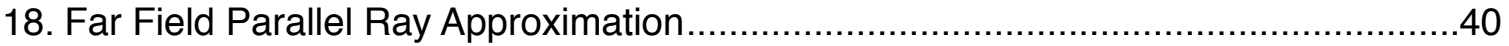

19. Polar Radiation Pattern Plot of the Narda 642 Horn Antenna at $5.5 \mathrm{GHz}$ as Measured by the Microwave Lab Chamber (Blue) and Hangar Lab 
Chamber (Red), and Predicted 1st and 2nd Nodes (Green) and Nulls (Black)

20. Polar Radiation Pattern Plot of the Narda 643 Horn Antenna at $5.0 \mathrm{GHz}$ as Measured by the Microwave Lab Chamber (Blue) and Hangar Lab Chamber (Red), and Predicted 1st and 2nd Nodes (Green) and Nulls (Black)

21. Polar Radiation Pattern Plot of the Broadband Horn Antenna at $3.0 \mathrm{GHz}$ as Measured by the Microwave Lab Chamber (Blue) and Hangar Lab Chamber (Red), and Predicted 1st and 2nd Nodes (Green) and Nulls (Black)

22. A Double Ridged Broadband Horn Antenna 45

23. Polar Radiation Pattern Plot of the $915 \mathrm{MHz}$ Patch Antenna at $915 \mathrm{MHz}$ as Measured by the Microwave Lab Chamber (Blue) and Hangar Lab Chamber (Red) 46

24. Power vs Azimuth Scatter Plot of the Narda 642 Horn at $5.5 \mathrm{GHz}$ as Measured by the Microwave Lab Chamber (Blue) and Hangar Lab Chamber (Red)

25. XY Scatter Plot of Narda 642 Horn Antenna Pattern Measured in Both Chambers with Noise Floor, Nodes and Nulls .....

26. XY Scatter Plot of Narda 643 Horn Antenna Pattern Measured in Both Chambers with Noise Floor, Nodes and Nulls .52

27. XY Scatter Plot of $915 \mathrm{MHz}$ Patch Antenna Pattern Measured in Both Chambers with Noise Floor. .53

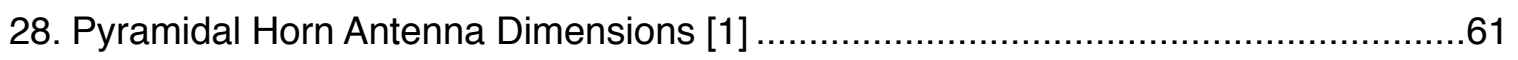

29. 2.4 GHz Pyramidal Horn Model in HFSS ...................................................63 


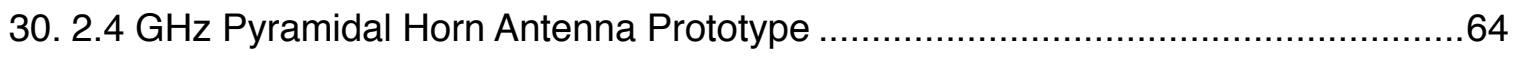

31. S11 Log Magnitude Plot Comparing HFSS Model to Prototype ............................65

32. Normalized Rectangular Logarithmic Radiation Pattern Comparing

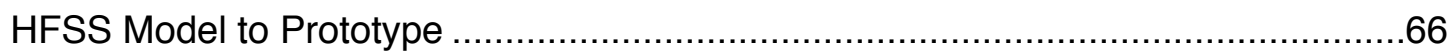

33. Geometry for Field Calculations of a Line Source .......................................... 71

34. Parallel Ray Approximation for Far-Field Calculations of a Line Source ...................72 


\section{CHAPTER 1: Introduction}

\subsection{Why Test Antennas?}

All wireless systems rely on antennas to transmit and receive electromagnetic messages. Depending on the communication system - GPS, Wi-Fi, or point-to-point data links - antennas are used to optimize message transmission for multiple systems. It is important to test antennas to verify system-required performance standards including gain and directivity. Gain and directivity are defined along with other antenna fundamentals in section 1.3.

Characteristics of antenna performance include radiation pattern, resonant frequency, and sensitivity as part of a system. Measurements must be conducted in an isolated, noise-free environment. Electromagnetic noise prohibits accurate antenna measurement and is attenuated by an anechoic chamber discussed in the following section.

Antenna testing involves repetitive measurements while rotating the antenna under test (AUT) in all directions, and this lends itself to automation. Measuring the gain of an antenna in a single dimension is simple to do manually, but to manually measure its gain in a $360^{\circ}$ spherical plane at $1^{\circ}$ intervals becomes time consuming and difficult to perform accurately. Automating the measurement therefore improves the design process by decreasing test duration and improving the quality of results. Furthermore, automation improves test repeatability and eliminates many possibilities for human error.

The results presented in this paper contribute to the sphere of antenna measurement by providing new insight into the benefits and drawbacks of using smaller anechoic chambers over larger ones. 


\subsection{Why an Anechoic Chamber?}

Antennas are tested in what are known as antenna ranges, and while various types of ranges exist, each strive to create a similar ideal test environment. The two most important environmental variables in antenna testing are noise and reflections. Noise is undesirable electromagnetic interference that can come from various sources both external and internal to the antenna system. Reflections deflect the test environment surfaces and superimpose with the direct path signal.

Anechoic chambers are enclosed rooms lined with absorber material designed to suppress reflections. While more expensive than outdoor ranges, anechoic chambers offer isolation from the outside world and an enclosed and consistent test environment. Outdoor ranges suffer exposure to ambient RF signals and weather limitations. Weather is unpredictable, affects the characteristics (humidity and rain) between the transmit and receive antennas, and can damage test equipment.

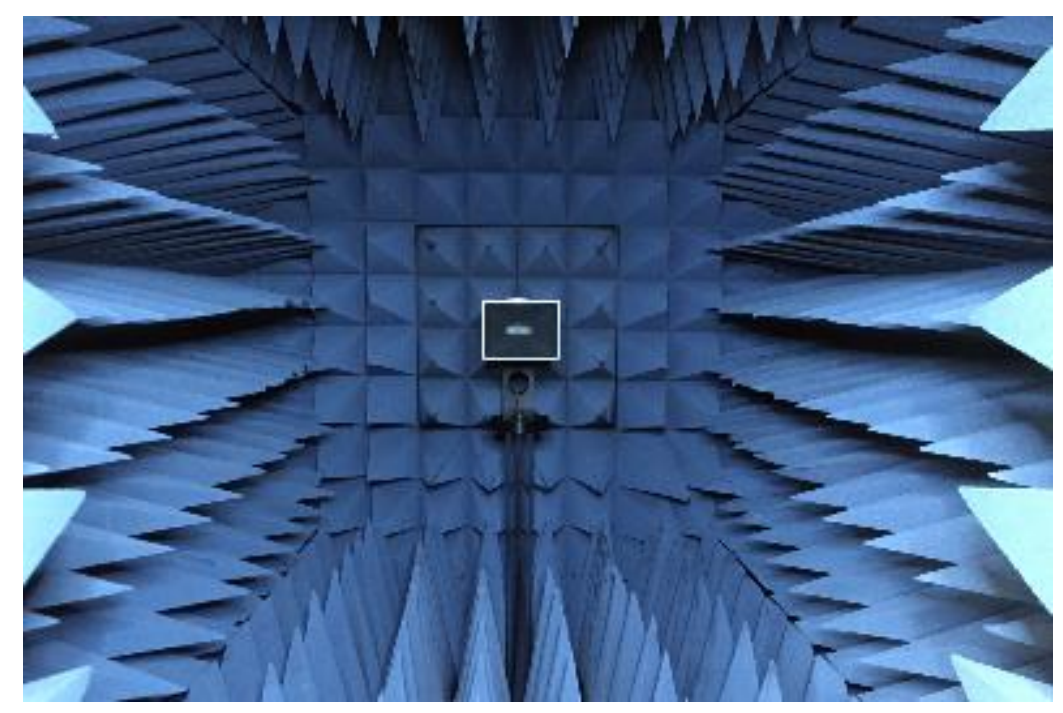

Figure 1 Pyramidal Horn Antenna in the Cal Poly Microwave Lab Anechoic Chamber 
The Cal Poly Microwave Lab anechoic chamber in Figure 1 uses pyramidal foam blocks to absorb stray signals and suppress reflections. Pyramidal absorbers progressively absorb energy through ohmic losses by steadily increasing the impedance from free space at the surface of the absorber to high impedance lossy material.

In addition to testing antennas, anechoic chambers are commonly used for FCC emissions and immunity testing on cell phones. The FCC sets guidelines for what power and frequency signals devices can emit to not interfere with other wireless systems. Immunity testing ensures that a system can operate normally where foreign RF sources are present. The noise free environment is ideal for the scientific accuracy of these tests.

\subsection{Antenna Fundamentals}

The following section defines antenna radiation patterns, gain, polarization, and common measurement methods. Section 1.3.5 defines near and far field regions and how they affect anechoic chamber size and operating frequency range. 


\subsubsection{Antenna Coordinate System}

Engineers use the antenna spherical coordinate system in Figure 2 to define antenna position and properties. Phi rotation about the z-axis is azimuth, and theta rotation away from the z-axis is elevation. In an anechoic chamber, the $\mathrm{x}$-axis is defined with respect to the chamber and points from the antenna under test to the receive antenna.
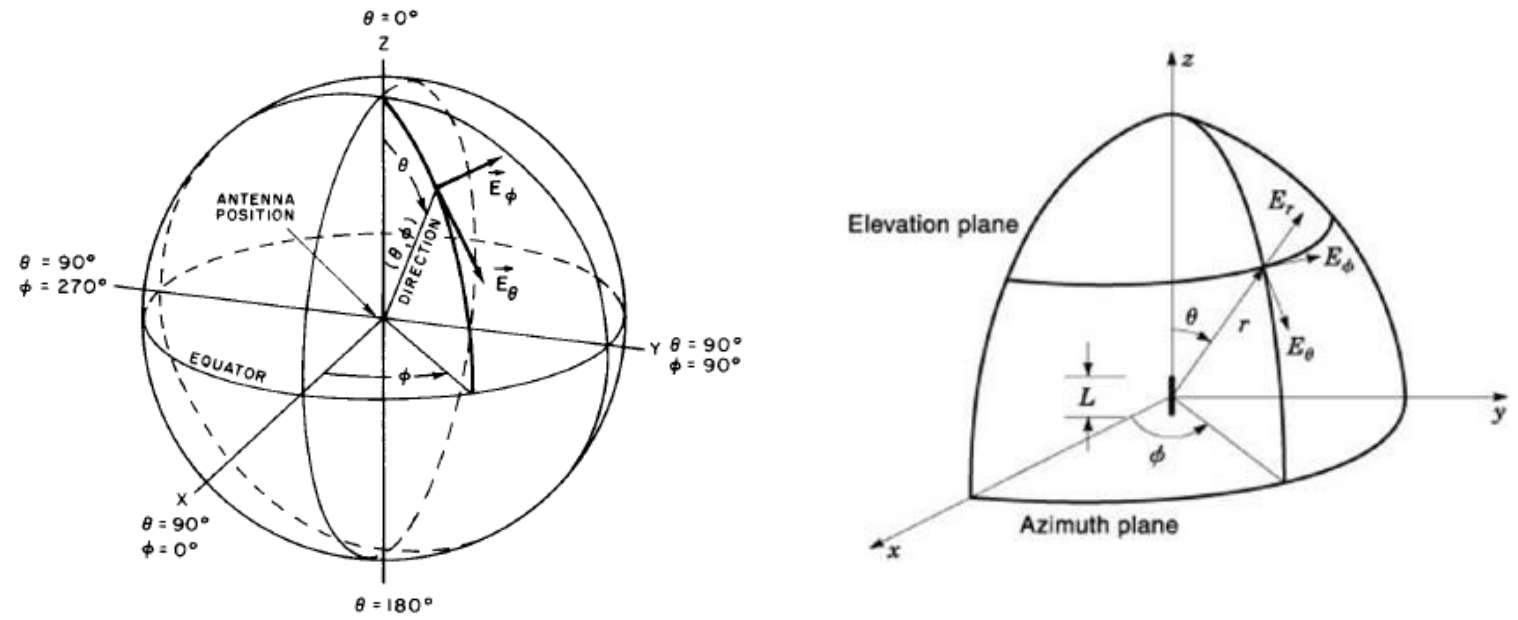

Figure 2 Antenna Spherical Coordinate System [2, 9]

\subsubsection{Radiation Patterns}

"An antenna [radiation] pattern is a graphical representation of the field magnitude at a fixed distance from an antenna as a function of direction. With the antenna at the origin of a spherical coordinate system, radiation fields $E$ and $H$ are perpendicular to each other and both are transverse to the direction of propagation" [1]. Half-power beam width (HPBW), gain, directivity, and polarization are performance factors derived from pattern measurements and are discussed in sections 1.3.3 and 1.3.4. 
The normalized field pattern is a polar plot of field magnitude normalized relative to the maximum radiated E-field. The main beam is the direction the antenna concentrates most of its radiation; side lobes are weaker radiation lobes in other directions. $H P B W$ is the angular span between the two half-power $(-3 \mathrm{~dB})$ points on either side of the main beam.

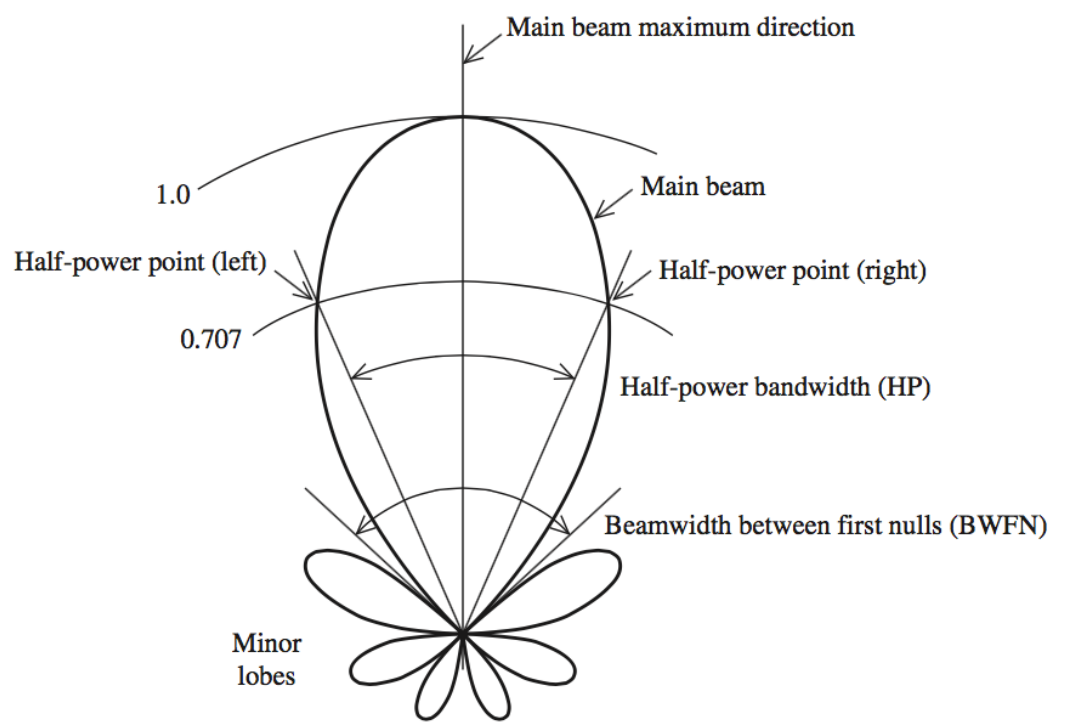

Figure 3 Normalized Radiation Field Pattern and its Components [1]

Measured radiation patterns are often plotted in decibels. The logarithmic power pattern is a polar plot of these values and relates to the absolute field pattern as follows.

$$
P(\theta, \phi)_{\mathrm{dB}}=10 \log P(\theta, \phi)=10 \log |F(\theta, \phi)|^{2}=20 \log |F(\theta, \phi)|=|F(\theta, \phi)|_{\mathrm{dB}} \quad \text { Equation } 1
$$

where $\mathrm{P}(\theta, \phi)(\mathrm{dB})$ is the logarithmic power pattern and $\mathrm{F}(\theta, \phi)$ is the absolute field pattern. Radiation patterns are typically plotted as logarithmic power patterns to show more detail in the side lobes. 
To measure radiation pattern, an RF probe is placed in the far field of the AUT. While the AUT rotates through all its spherical angles, a constant signal is transmitted from the antenna, and power levels received by the probe are recorded for each angle. Alternatively, the AUT can remain stationary while the probe moves around the antenna at a constant radius. The probe is a calibrated antenna connected to an RF power meter. In addition to rotation, both the AUT and RF probe positioners include a roll axis to enable polarization testing, see Figure 4. Polarization is described in section 1.3.4.

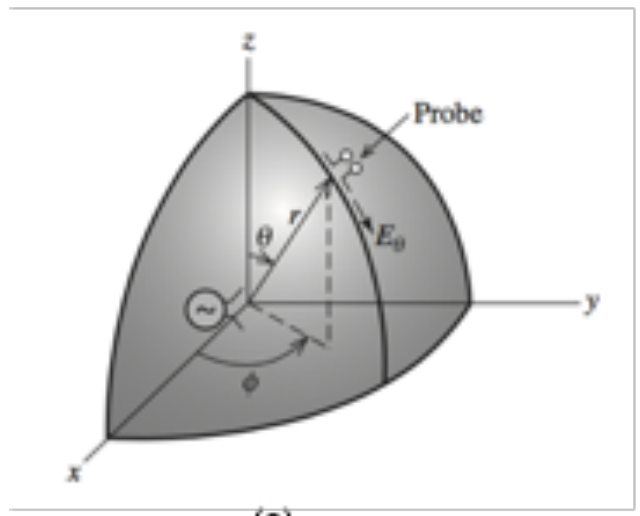

(a)

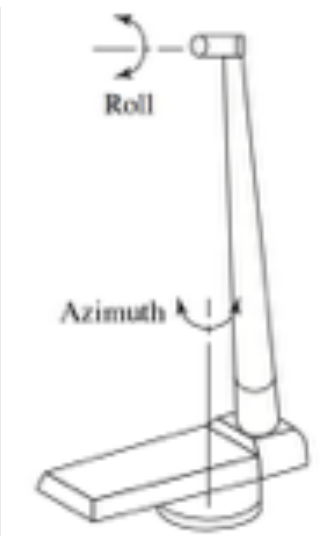

(b)

Figure 4 (a) Pattern Measurement Conceptualized (b) Model Positioner [2]

In practice, a vector network analyzer (VNA) can be the sole instrument in an antenna measurement system. A VNA is used to measure two-port systems by sending a signal into one port and measuring what is received at the other port. It can both generate the signal transmitted by the AUT and measure the received signal by the RF probe. It is also capable of performing a separate reflection measurement on the AUT over a frequency range to determine what frequency the antenna transmits best. Any power not transmitted by an antenna is reflected back to the RF generator, or VNA in this case. 
In Figure 5 below, the black box represents a two-port system. The left side is port 1 and the right side is port 2. The VNA also has two ports, each connected to one side of the system. A VNA measures scattering parameters (S-parameters) of a system through incident, reflected, and transmitted signals. Signal $a_{1}$ is the component of the signal generated by the VNA that enters the system and is incident on port 1, while signal $b_{1}$ is the component reflected by port 1 . Signal $b_{2}$ is the component transmitted from port 1 to port 2 and received by the VNA, and signal $a_{2}$ is the component transmitted from port 1 to port 2 but reflected at the VNA. $S_{11}$ is the ratio of the reflected signal to incident signal at port 1 , the reflection coefficient of port $1 . S_{22}$ is identical to $S_{11}$ but at port 2. $S_{21}$ is the ratio of the signal transmitted to port 2 from port 1 , the forward gain. $S_{12}$ is the opposite of $S_{21}$ known as the reverse gain. The VNA sweeps outgoing signals over a frequency range, and displays S-parameters as a function of frequency.

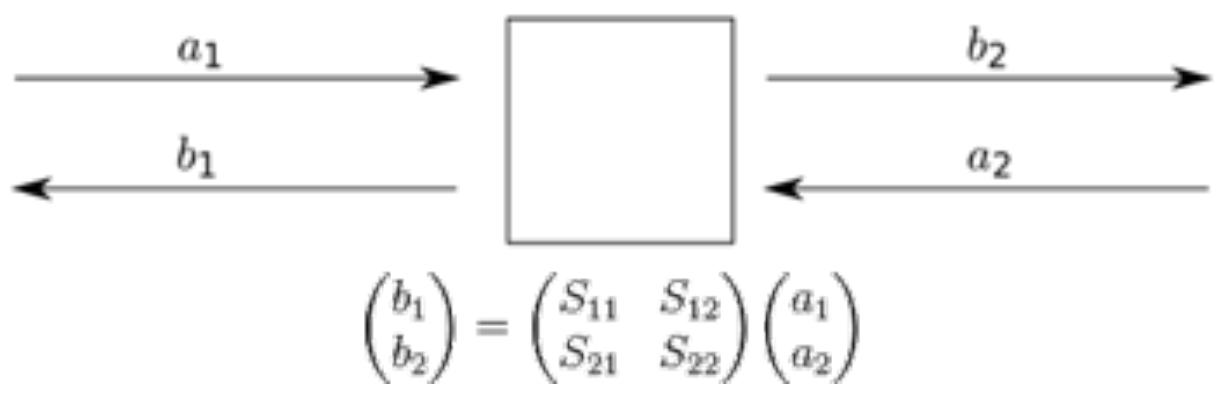

Figure 5 Illustration of S-parameters for a Two-Port System

For an antenna connected to port $1, S_{11}$ will be close to unity over the frequencies which the antenna does not radiate well, and small over the frequencies which do radiate. This AMS delivers a signal to the AUT on port 1, and the signal is sent over the air to the known antenna on port 2. $S_{21}$ is the forward gain of the antenna-probe system, or the ratio of the signal sent to the AUT versus the signal received by the known antenna. The loss through the cables connecting the AUT and probe to the VNA 
is negligible, $<1 \mathrm{~dB}$, because they are impedance matched to the VNA $(50 \Omega)$. Therefore $S_{21}$ equal to the power sent to the AUT, minus the power reflected at the AUT input port, minus the air loss between the AUT and the probe, and minus the power not picked up by the probe. With an ideal RF probe, any power not picked up is power sent in other directions by the AUT. The air loss between the AUT and the probe is a constant, and can be accounted for by normalizing the $S_{21}$ data. Thus, the radiation pattern for the AUT is just $S_{21}$ plotted over all spherical angles.

\subsubsection{Directivity and Gain}
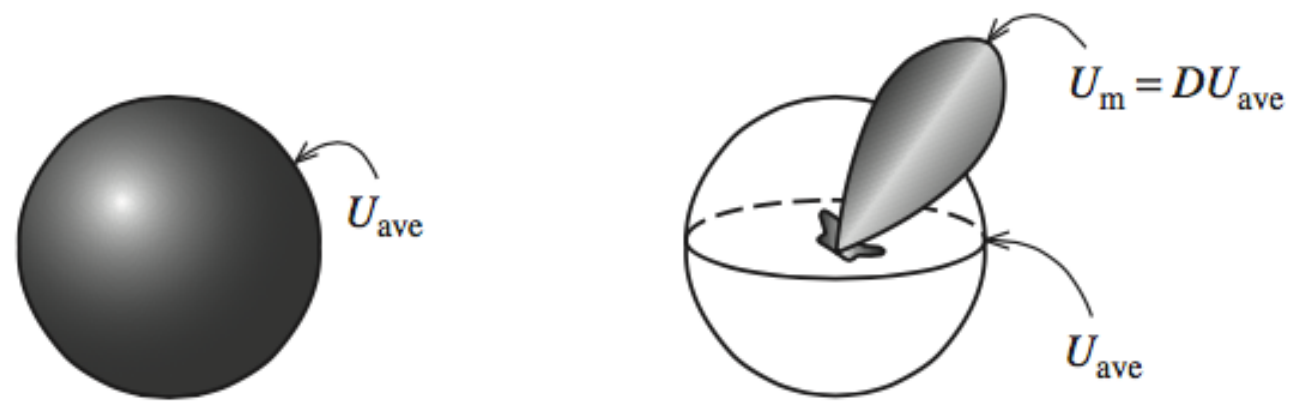

Figure 6 Illustration of Directivity [1]

An antenna's directivity (D) is the ratio of the radiation intensity in the main beam direction $\left(U_{m}\right)$ to the average radiation intensity $\left(U_{\text {ave }}\right)$. Radiation intensity is the power radiated in each direction per unit solid angle (steradian), and is independent of distance (r). The total power $(\mathrm{P})$ radiated by an antenna is the integral of the radiation intensity over all solid angles around the antenna. The directivity calculation compares an antenna's radiation pattern to that of an ideal uniform (isotropic) source. An isotropic source has uniform radiation intensity, but is unachievable in practice. $U_{\text {ave }}$ is the power radiated in a single steradian if the antenna pattern were isotropic. Since only $4 \pi$ steradians are in a sphere, $\mathrm{U}_{\text {ave }}$ is $\mathrm{P}$ divided by $4 \pi$. An isotropic source has the minimum possible directivity of 1 , and all real antennas have a directivity greater than 1 . 


$$
D=\frac{U_{m}}{U_{\mathrm{ave}}}=\frac{U_{m}}{P / 4 \pi}
$$

\section{Equation 2}

The gain of an antenna is the directivity multiplied by its radiation efficiency, where radiation efficiency is the ratio of total power transmitted $(P)$ to input power $\left(P_{\text {in }}\right)$. Many antennas are highly efficient with an efficiency close to one, so their directivity is a good approximation of their gain. However electrically small antennas, where physical dimensions are less than one half wavelength, are relatively inefficient and have efficiencies much less than one [1].

$$
G=e_{r} D \quad e_{r}=\frac{P}{P_{\text {in }}} \quad \text { Equation 3 }
$$

Antennas with directivities close to unity, meaning they transmit more or less equally in all directions, are commonly used in broadcast transmission and receiving so that reception is not dependent on the direction each party is facing. Examples are antennas used in cell phones and broadcast radio. High gain antennas, on the other hand, are capable of transmitting over longer distances with the same applied input power, are used in point-to-point communication systems. Another application of high gain antennas is receiving the signal of broadcast antenna from a further distance possible than with an isotropic receiver, provided that the direction of the source is known. Using advanced techniques it is also possible to design an antenna with an adjustable gain and main beam direction. This requires an array of antennas, where the separation distance between them is known, and the same signal is sent to each antenna but with a slightly different phase such that their mutual radiations add and subtract in what is known as beam forming, or beam steering. 


\subsubsection{Polarization}

Electromagnetic radiation from an antenna is composed of coupled electric (E) and magnetic $(\mathrm{H})$ fields that oscillate in magnitude over time and space. As an electromagnetic wave propagates, its $\mathrm{E}$ and $\mathrm{H}$ field vectors oscillate and rotate about the direction of propagation in an unchanging pattern. This pattern, called wave polarization, is a product of the shape of the antenna that generated them. Generally, antennas can only receive signals that match their own polarization.

Figure 7 shows the dual field vectors of a linearly polarized wave propagating along the z-axis at a fixed moment in time. Polarization can be described as the behavior of only the $\mathrm{E}$ field vector as the wave propagates because the $\mathrm{H}$ field will always be orthogonal. Because the electric field vector in Figure 7 only oscillates up and down as it travels along the z-axis, and does not rotate, the wave is considered linearly polarized. Because the electric field vector oscillates up and down the x-axis, and not side to side with the $y$-axis, the wave is vertically polarized. Figure 7 also shows how the electric and magnetic field vectors are orthogonal in the direction of propagation.

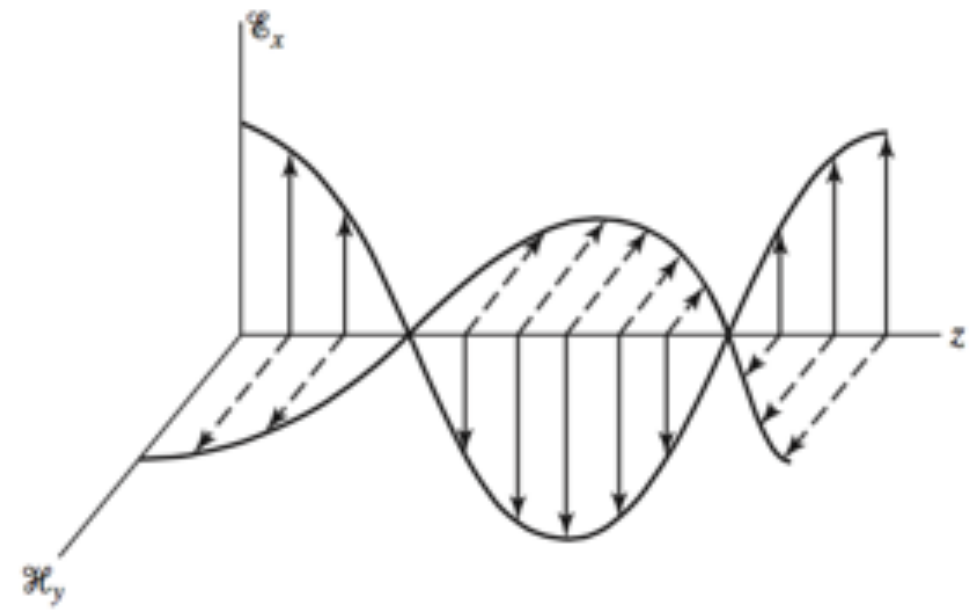

Figure 7 Spatial Behavior of Electric (solid) and Magnetic (dashed) Fields of a Linearly (vertical) Polarized Wave for a Fixed Instant of Time [1] 
Typically, polarization is illustrated on an XY plot showing the behavior of just the electric field vector along the axis of propagation. Figure 8 illustrates some wave polarization states. Generally, waves have an elliptical polarization with an $\mathrm{x}$ and $\mathrm{y}$ component and either a left-hand or right-hand sense of rotation. However, dipole and monopole antennas are considered linearly polarized - a special case of elliptical polarization.

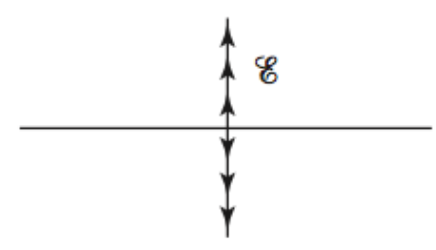

(a) Vertical linear polarization.

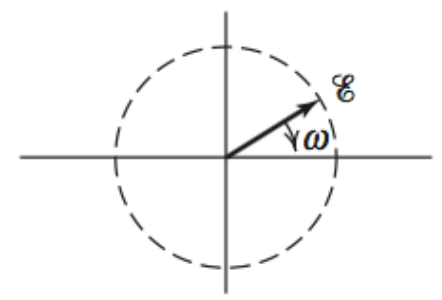

(c) Left-hand circular polarization.

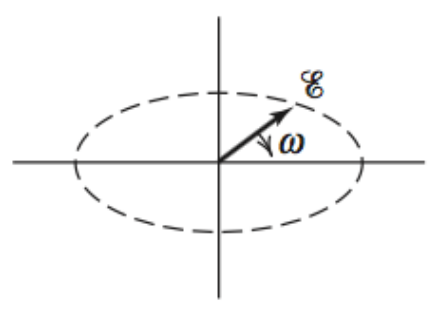

(e) Left-hand elliptical polarization.

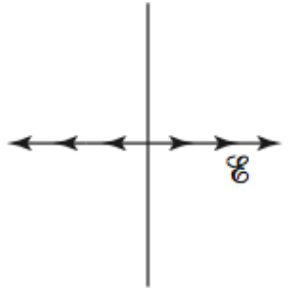

(b) Horizontal linear polarization.

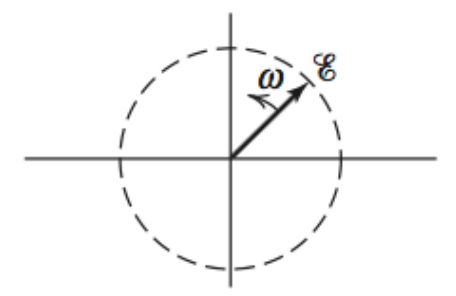

(d) Right-hand circular polarization.

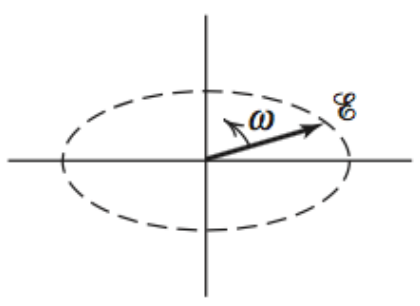

(f) Right-hand elliptical polarization.

Figure 8 Some Polarization States (the wave is approaching) [1] 
Another way to illustrate polarization is the Poincaré Sphere in Figure 9. The Poincaré Sphere is a 3D plot where all possible polarizations lie somewhere on its surface. Its equator represents all possible linear polarizations, its north pole represents perfect right-hand circular polarization, and its south pole represents perfect left-hand circular polarization. All other points on its surface represent the various elliptical polarizations.

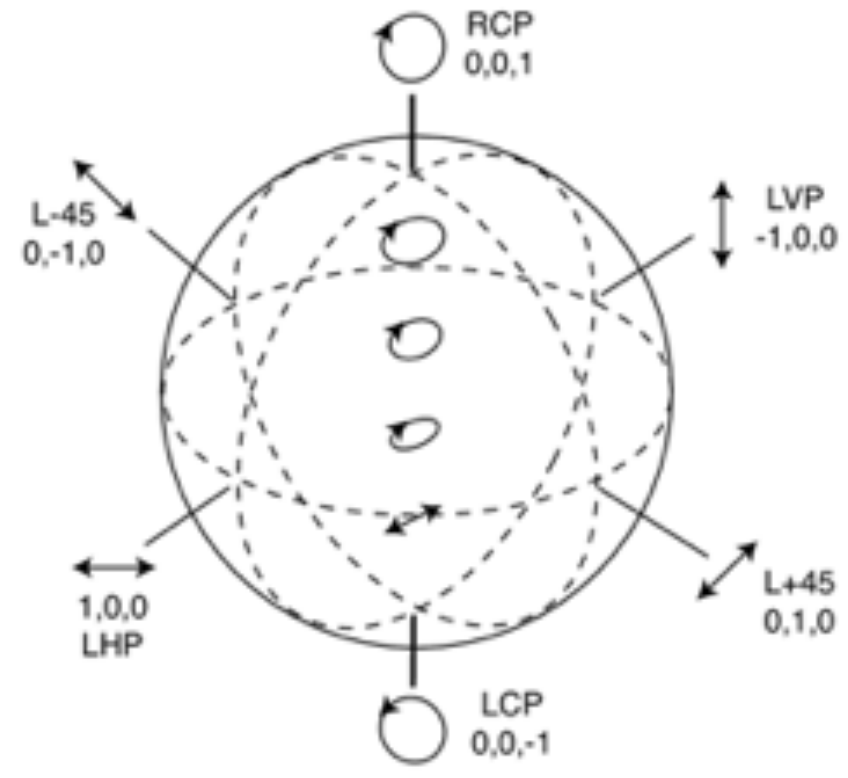

Figure 9 The Poincaré Sphere [1]

In the real world, antennas will emit multiple polarization types because they are not ideal, nonetheless the dominant polarization type defines the overall polarization. Antennas only receive signals that align with their polarization. This means a linear vertical polarized (LVP) antenna can only pick up signals with a vertical component. This includes elliptically polarized signals, where the ratio of vertical to horizontal components is proportional to the signal strength received. A linearly polarized antenna can receive circular polarized (CP) signals no matter its orientation. An example of the is GPS 
satellites which transmit CP signals so GPS receiving devices can pick up signals regardless of their orientation.

\subsubsection{The Near and Far Field}

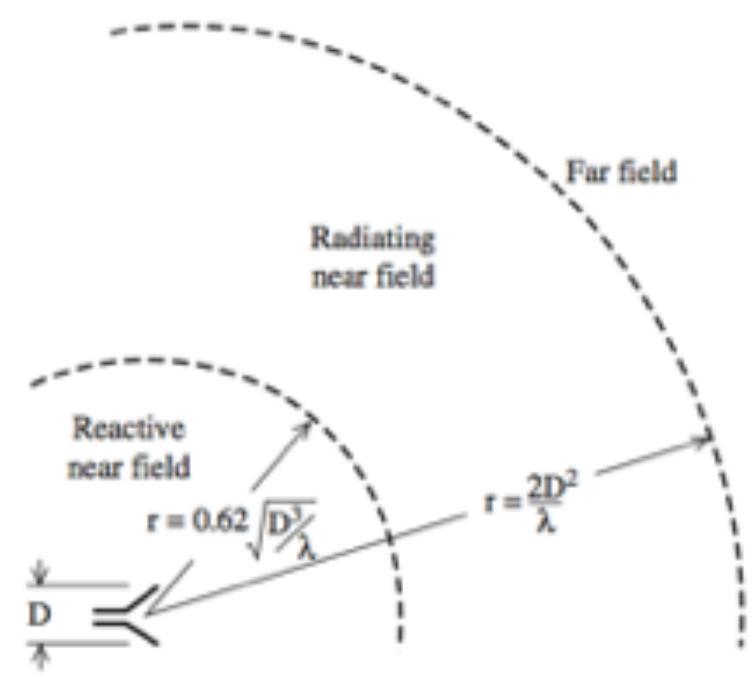

Figure 10 Summary of the Field Regions (not to scale) [1]

The near and far fields are regions surrounding a transmit antenna which describe the radiative/non-radiative nature of the EM signals within them. Closest to the antenna, reactive EM fields dominate in the reactive near field. These EM fields diminish greatly as they move away from the source antenna, at a $1 / r^{\wedge} 3$ rate, and are not suitable for long distance communication. Outside of the near field, radiative EM fields dominate. Radiative fields diminish much more gradually over greater distance from the source, at a $1 / r$ rate. The total radiative field energy passing through a surface at a distance $r$ is proportional to $1 / r^{\wedge} 2$. Since the area of a sphere is proportional to $r^{\wedge} 2$, the total energy passing through a sphere of any radius $r$ remains constant. This means that the far field energy escapes to infinite distance, i.e. it radiates. 
The radiating region is divided into the radiative near field and the far field. Due to the geometry of the antenna and the wavelength of the carrier frequency, waves in the radiative near field can be out of phase and incoherent to receivers. It is not until the waves pass the Fraunhofer distance that they are in the far field and an antenna can reliably receive them. Beyond this distance, the wave is approximately planar. While it is possible to design antennas for the near field, most antennas communicate in the far field. The Fraunhofer distance is given by:

$$
\mathrm{r}=\frac{2 \mathrm{D}^{2}}{\lambda}
$$

Equation 4

See Appendix A for the far field derivation.

The total far field energy passing through a surface at a distance $r$ from the source remains constant for any $r$. This means far field energy radiates to infinite distance if nothing blocks or absorbs it along the way. The ratio of the power received $\left(P_{r}\right)$ by an antenna to the power sent from a transmit antenna $\left(P_{t}\right)$ in the far field is given by the Friis equation.

$$
\frac{P_{r}}{P_{t}}=G_{t} G_{r}\left(\frac{\lambda}{4 \pi R}\right)^{2}
$$

$\mathrm{G}_{\mathrm{t}}$ and $\mathrm{G}_{\mathrm{r}}$ are the gain of the transmit and receive antennas, $\mathrm{R}$ is the distance separating the antennas, and $\lambda$ is the wavelength of the signal. The Friis equation is valid for radii $R$ greater than the longer near field distance of either antenna. Because gain is about equal to directivity, knowing and being able to measure directivity allows an RF system engineer to calculate the power needed to transmit a signal over a given distance. This is extremely important to RF system design and also the goal of this antenna measurement system project. 


\section{CHAPTER 2: Anechoic Chamber and Test Equipment}

This chapter describes the physical design of the new anechoic chamber, as well as the selection process for the antenna positioners and network analyzer.

\subsection{The Microwave Lab Anechoic Chamber}

The new anechoic chamber in the Cal Poly Microwave Lab measures 6'x6'x8' and is made of an interlocking wood frame. 16" pyramidal foam absorbers line the interior of the chamber except for a small opening at the bottom cut for the AUT mount. There are removable access panels on the ends, top, and sides of the chamber. The panels allow access to the AUT during mounting, provide a window for the receive antenna to measure the AUT from, and allow viewing of the AUT during a test to ensure the positioner is operating properly. Figure 11 shows a cross section of the chamber during a typical radiation pattern measurement test. 


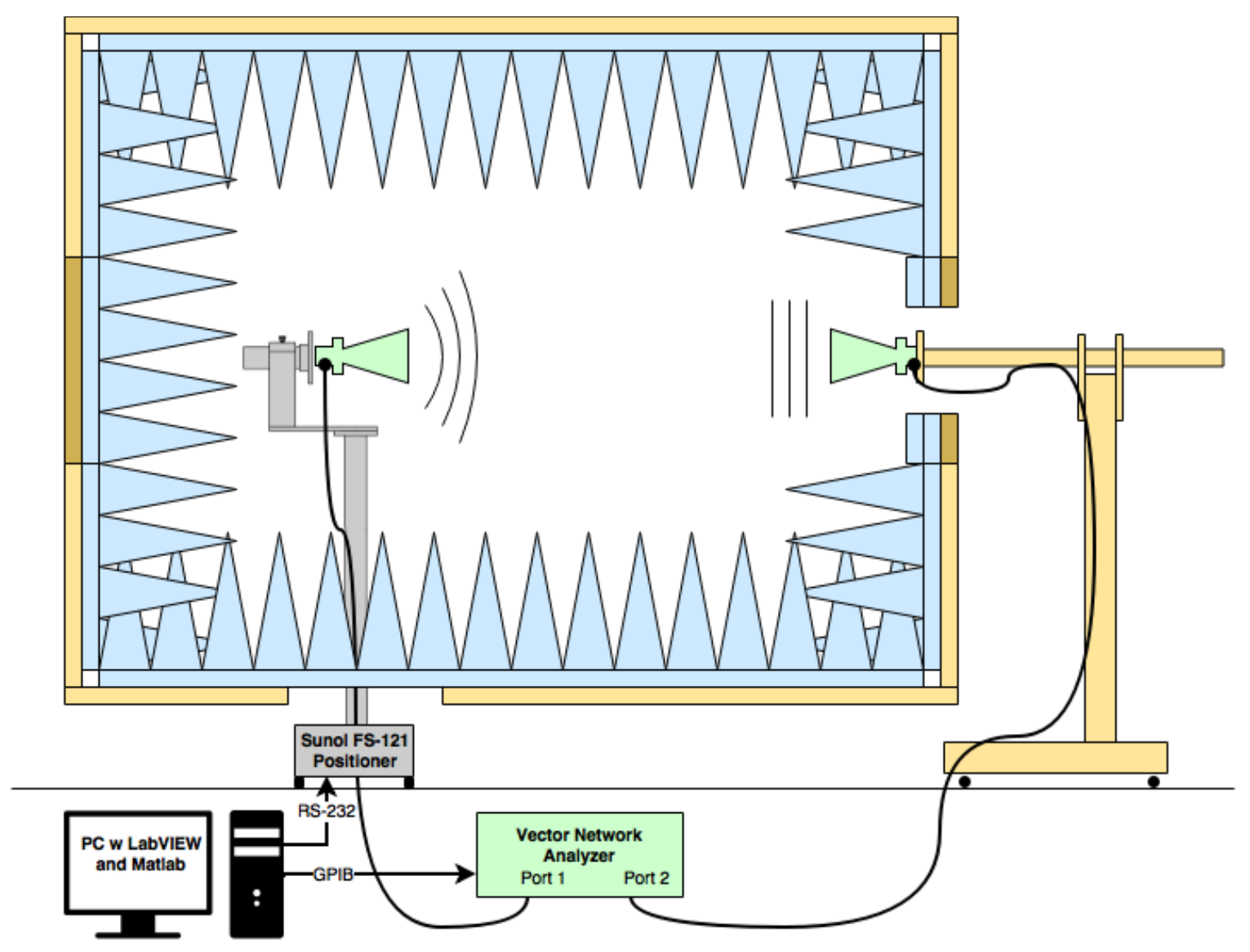

Figure 11 Cross Section of the Cal Poly Microwave Lab Anechoic Chamber (to scale)

Section 1.3.2 discussed two methods for antenna pattern measurement: rotating the AUT, or rotating the RF probe about the AUT. The former method works well for small scale setups like this one where the AUT/DUT is easy to rotate, while the latter method requires more space and equipment to move the RF probe around the AUT. Because this chamber is small, any antenna tested in it should be small and easy to rotate, also the hole in the bottom is the perfect size for an antenna positioner. To support these types of measurements the AMS has one programmable positioner and one manually adjustable positioner to allow a variety of physical test setups while keeping costs low. An RF test equipment manufacturer supplied the programmable positioner, while the manual positioner was designed and built on site to complement it. The programmable 
positioner can rotate the AUT over $360^{\circ}$ azimuthal sweeps. More on the positioner in the following sections. High frequency coaxial SMA cables link the VNA to the antennas, while a PC running LabVIEW and Matlab controls the instruments and generates reports.

\subsection{Choosing an AUT Positioner}

The important attributes of an antenna positioner include rotation type (azimuth, elevation, skew), load capacity (power, torque, angular accuracy), computer controllability, antenna mounting style, and total cost. Other considerations include durability, lifespan, and quality of customer support. Factors for this specific project include budget, size of the anechoic chamber, size of antennas under test, and the test automation software. The original goal was to stay under $\$ 2,000$ dollars.

It would be convenient to have more than one axis of rotation, but online research indicated that an azimuth only positioner would be the most cost effective. Azimuth is the main degree of freedom necessary for radiation pattern measurement, and more degrees of freedom tend to cost more money. Also, with the proper mount in an azimuthal positioner, the antenna can roll left or right manually to allow elevation sweeps or varied polarization. Furthermore, the addition of a motorized roll axis would add extra size and complexity. Because the motor produces noise and can interfere with the signals inside the chamber, it requires a gear and belt drive to stay outside the chamber.

The size of the anechoic chamber limits the size of the antennas that can fit inside and therefore provides an upper limit on the reasonable amount of power and torque necessary from the positioner motor. Chamber dimensions prohibit an antenna larger than $1 \mathrm{~m}^{3}$, as the antenna will need to fully rotate without touching the foam 
absorbers. The goal for max antenna weight is $50 \mathrm{lbs}$. These dimensions limit half wavelength antennas to frequencies above $150 \mathrm{MHz}$.

LabVIEW works with a wide variety of standard interfaces including USB, GPIB, and RS-232/Serial. Most commercial programmable instruments have LabVIEW drivers available online on the National Instruments website. Sometimes, however, these drivers do not exist and must be custom made. There is a tradeoff between cost and the ease of use of pre-written drivers. On one hand, making use of a positioner with a pre-made computer interface requires less work. On the other hand, it is inexpensive to write drivers. Additionally, one can write custom drivers tailored to the project's specific needs.

Finally, the positioner should be durable and last at least 10 to 20 years without excessive repair or replacement. Students are clumsy and this positioner should be sufficiently sturdy to withstand their mistakes. The product supplier should also be sufficiently reliable to provide support and replacement parts for years to come. Table 1 below and on the following page compares six potential positioners with these considerations in mind. 
Table 1 Potential Antenna Positioners and their Features

\begin{tabular}{|c|c|c|c|c|c|}
\hline Company & Model & $\begin{array}{l}\text { Torque/ } \\
\text { Power/Load } \\
\text { Capability }\end{array}$ & $\begin{array}{l}\text { Computer } \\
\text { Connectivity }\end{array}$ & $\begin{array}{l}\text { Mast/Antenna } \\
\text { Mount }\end{array}$ & $\begin{array}{l}\text { Total } \\
\text { Cost }\end{array}$ \\
\hline $\begin{array}{l}\text { Sunol } \\
\text { Sciences }\end{array}$ & $\begin{array}{l}\text { ELAZ75 } \\
\text { Elevation/ } \\
\text { Azimuth } \\
\text { Antenna } \\
\text { Positioner } \\
\text { System }\end{array}$ & $\begin{array}{l}\text { Manufacturer } \\
\text { did not } \\
\text { respond }\end{array}$ & $\begin{array}{l}\text { RS-232 } \\
\text { included } \\
\text { No LabVIEW } \\
\text { drivers }\end{array}$ & Included & $\$ 15,000$ \\
\hline $\begin{array}{l}\text { Sunol } \\
\text { Sciences }\end{array}$ & $\begin{array}{l}\text { FS-121 Free } \\
\text { Space } \\
\text { Turntable }\end{array}$ & $\begin{array}{l}\text { EUT Load } \\
\text { Rating 10lb }\end{array}$ & $\begin{array}{l}\text { RS-232 } \\
\text { included } \\
\text { No LabVIEW } \\
\text { drivers, but } \\
\text { simple serial } \\
\text { commands } \\
\text { available }\end{array}$ & $\begin{array}{l}\text { Custom Mast } \\
\text { Available }\end{array}$ & $<\$ 3,000$ \\
\hline Yaesu & $\begin{array}{l}\text { G-800DXA } \\
\text { Amateur } \\
\text { antenna } \\
\text { positioner } \\
\text { motor }\end{array}$ & $\begin{array}{l}20 \text { sq. ft. } \\
\text { antennas } \\
\text { medium duty }\end{array}$ & $\begin{array}{l}\text { RS-232 } \\
\text { computer } \\
\text { interface sold } \\
\text { separately } \\
\$ 590 \\
\text { No LabVIEW } \\
\text { drivers }\end{array}$ & $\begin{array}{l}\text { Mast not } \\
\text { included } \\
\text { Antenna } \\
\text { bracket sold } \\
\text { separately } \\
\$ 36 \\
\text { Vibration plate } \\
\$ 150\end{array}$ & $<\$ 1,500$ \\
\hline M2 Inc. & $\begin{array}{l}\text { OR2800PXA } \\
\text { Z }\end{array}$ & $\begin{array}{l}4200 \text { in Ib. } \\
\text { very heavy } \\
\text { duty }\end{array}$ & $\begin{array}{l}\text { RS-232 } \\
\text { included } \\
\text { No LabVIEW } \\
\text { drivers }\end{array}$ & $\begin{array}{l}\text { Mast and } \\
\text { bracket not } \\
\text { included }\end{array}$ & $\$ 2,600$ \\
\hline $\begin{array}{l}\text { Anaheim } \\
\text { Automation }\end{array}$ & $\begin{array}{l}\text { 23MDSI306 } \\
\text { D-04 }\end{array}$ & $\begin{array}{l}15 \text { in lb. } \\
\text { light duty }\end{array}$ & $\begin{array}{l}\text { RS-485 } \\
\text { RS-232 } \\
\text { converter } \\
\text { sold } \\
\text { separately } \\
\$ 140 \\
\text { No LabVIEW } \\
\text { drivers }\end{array}$ & $\begin{array}{l}\text { Mast and } \\
\text { bracket not } \\
\text { included }\end{array}$ & $\$ 400$ \\
\hline
\end{tabular}




\subsection{The FS-121 Positioner}

Even though it exceeds the planned budget, the FS-121 Azimuthal Positioner from Sunol Sciences combines the best features with the best price. It communicates with easily programmed ASCII serial commands. It is accurate and rated to $<1^{\circ}$ resolution and repeatability with a 10-lb. load limit. Compared to less expensive options, it requires no assembly, stands on a single component with a small footprint, and has the robustness of a more expensive unit. Extra funding from the EE department made it possible to purchase the positioner with money left over. Sunol Sciences is a small company located in Dublin, CA that manufactures positioning equipment and antennas for EMC and wireless testing. The EE department has ordered from them in the past for its 20' anechoic chamber, and they offer helpful customer support.

The FS-121 is a floor unit with interchangeable acrylic masts to support a variety of antennas. Figure 12 shows the two masts included with the FS-121: a custom mast with antenna fixture and a standard mast with turntable. The custom fixture rolls either direction for antenna polarization, and the center of the roll axis is at chamber mid height 50 " above the floor. The fixture secures antennas on a circular flange with eight 9/32" diameter mounting holes, spaced $45^{\circ}$ apart in a $5^{\prime \prime}$ diameter. Wooden adaptors facilitate flange-mounting antennas. See Appendix B for custom fixture and flange schematics. 

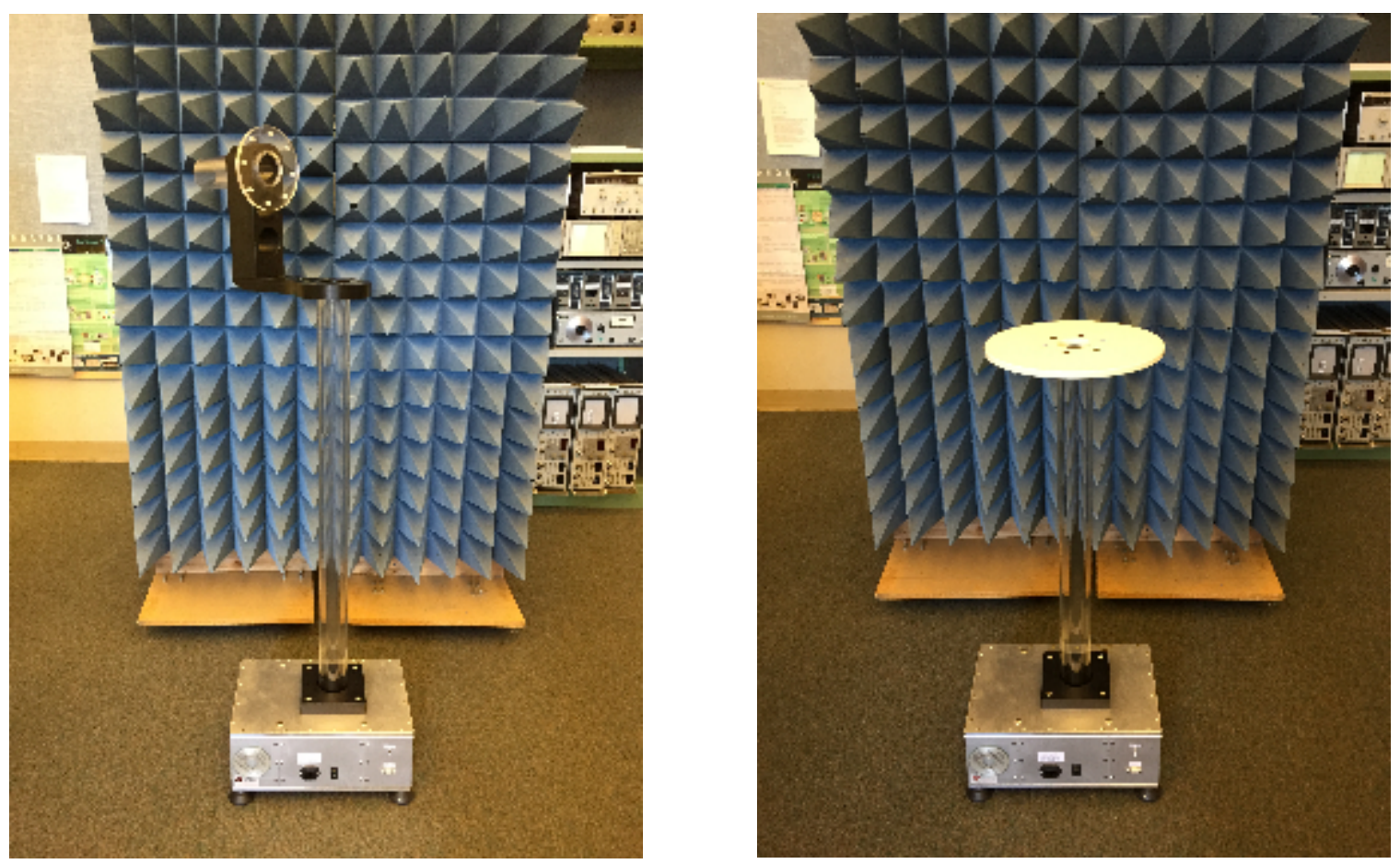

Figure 12 FS-121 Positioner with Custom Mast (Left) and Turntable (Right)

While the custom mount securely holds antennas at chamber mid-height, if an antenna exceeds its size or weight ratings $\left(1 \mathrm{~m}^{3}, 10 \mathrm{lbs}\right.$.) it can wobble when steprotating. In this case, it is important to check that excessive wobbling does not occur, thus ruining the accuracy of the test. Larger antennas are not recommended for this reason. However, for larger antennas, or antennas not compatible with the flange mount, one should modify the turntable to hold them. Correctly securing antennas to the fixtures designed for the chamber increases test accuracy and repeatability. Securing antennas with nylon nuts and bolts, as opposed to steel or aluminum, prevents the hardware from interfering with the test. 


\subsection{Stationary Antenna Fixture}

The custom stationary antenna fixture, or non-remote controlled fixture, securely holds antennas up to $20 \mathrm{lbs}$. Only the chamber opening (24" x 24") limits antenna size. The wooden fixture has minimal EM interference, and caster wheels enable easy movement. A 2" diameter dowel supports the mount flange and can roll to change polarization as well as move in and out of the mast fixture to alter antenna separation distance. The mount flange has the same hole pattern as the AUT fixture and wood glue with a single wood screw fixes it to the dowel. Design schematics are available in Appendix C.

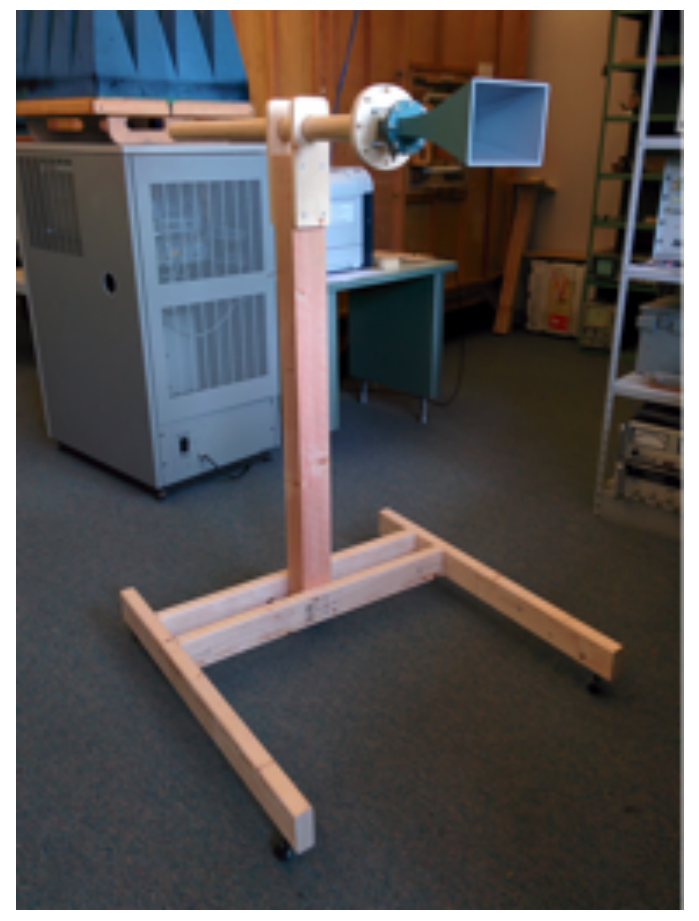

Figure 13 Receiver Fixture with Horn Antenna 


\subsection{Vector Network Analyzers}

The Microwave Lab has six Anritsu VNAs designated for teaching labs and three multipurpose HP network analyzers with S-parameter test sets. Ultimately, the VNA determines the operating frequency range of the system. Wider bandwidth VNAs are desirable. Table 2 lists Cal Poly's VNAs and their availability for this project.

Table 2 VNA Models, Frequency Ranges, and Availability

\begin{tabular}{|l|l|l|l|l|}
\hline Make and Model & \multicolumn{1}{|c|}{$\begin{array}{l}\text { Count } \\
\text { Anrequency } \\
\text { Range }\end{array}$} & \multicolumn{2}{l|}{$\begin{array}{l}\text { Current Use } \\
\text { Calibration }\end{array}$} \\
\hline Anritsu MS4622B & 5 & $\begin{array}{l}10 \mathrm{MHz} \text { to 3 } \\
\mathrm{GHz}\end{array}$ & $\begin{array}{l}\text { Microwave Lab } \\
\text { Student Labs }\end{array}$ & Good \\
\hline Anritsu MS4623D & 1 & $\begin{array}{l}10 \mathrm{MHz} \text { to } 6 \\
\mathrm{GHz}\end{array}$ & $\begin{array}{l}\text { Microwave Lab } \\
\text { Student Labs }\end{array}$ & Good \\
\hline Anritsu MS4624D & 1 & $\begin{array}{l}10 \mathrm{MHz} \text { to } 9 \\
\mathrm{GHz}\end{array}$ & $\begin{array}{l}\text { Special } \\
\text { Applications }\end{array}$ & Good \\
\hline $\begin{array}{l}\text { Hewlett Packard } \\
\text { 8753ES }\end{array}$ & 1 & $\begin{array}{l}30 \mathrm{kHz} \text { to } 6 \\
\mathrm{GHz}\end{array}$ & $\begin{array}{l}\text { Microwave Lab } \\
\text { None }\end{array}$ & $\begin{array}{l}\text { Reference } \\
\text { Only }\end{array}$ \\
\hline $\begin{array}{l}\text { Hewlett Packard } \\
\text { 8753C }\end{array}$ & 1 & $\begin{array}{l}300 \mathrm{kHz} \text { to } 6 \\
\mathrm{GHz}\end{array}$ & $\begin{array}{l}\text { Microwave Lab } \\
\text { None }\end{array}$ & $\begin{array}{l}\text { Reference } \\
\text { Only }\end{array}$ \\
\hline $\begin{array}{l}\text { Hewlett Packard } \\
\text { 8753A }\end{array}$ & 1 & $\begin{array}{l}300 \mathrm{kHz} \text { to 3 } \\
\mathrm{GHz}\end{array}$ & $\begin{array}{l}\text { Microwave Lab } \\
\text { None }\end{array}$ & Good \\
\hline $\begin{array}{l}\text { Hewlett Packard } \\
\text { 8720D }\end{array}$ & 1 & $\begin{array}{l}50 \mathrm{MHz} \text { to 20 } \\
\mathrm{GHz}\end{array}$ & Hangar Lab & Good \\
\hline
\end{tabular}

The $20 \mathrm{GHz}$ HP model has the highest upper frequency limit, but it is currently exclusively used for Cal Poly's 20' anechoic chamber. Nevertheless, the $9 \mathrm{GHz}$ Anritsu has the bandwidth to accommodate most projects including those dealing with AM, FM, GSM, GPS, NFC, PCS, Wi-Fi, and Bluetooth. For versatility, the AMS software works with any of the Anritsu models, and the HP 8753ES. Because the code is modular, users can later add support for other models. More on the control software in the next chapter. 


\section{CHAPTER 3: LabVIEW Control System}

LabVIEW is a graphical programming language from National Instruments that uses graphical user interfaces (GUIs) called front panels to operate unseen code block diagrams. Each front panel and block diagram pair is known as a virtual instrument, or VI, and can call any number of other sub-VIs. LabVIEW controls instruments using the Virtual Instruments Software Architecture standard (VISA) and the Standard Commands for Programmable Instruments syntax (SCPI) over a variety of protocols including GPIB, Serial, USB, and Ethernet. Programming instruments remotely allows access to all normal functions of an instrument, with finer detail, and the ability to export data directly. Instrument drivers for LabVIEW are available for free from the National Instruments website www.ni.com. LabVIEW is the software of choice for test and measurement automation in the electrical engineering field. It's graphical interface makes it easy for new users, as well as the software engineer who programs it.

The AMS uses LabVIEW to calibrate the VNA, capture S-parameter data, rotate the antenna positioner, and save the data to files. LabVIEW also calls Matlab functions to graph S-parameter data of polar plots and evaluate HPBW and directivity. The top

level "AMS.vi" unites these features into a single user interface. Furthermore, this top VI compresses into a single executable file that can be run on any computer that has the free LabVIEW Run-Time Engine (RTE) and National Instruments VISA drivers.

The AMS GUI organizes information into four tabs alongside a panel for status and connectivity settings/indicators. The four tabs include: Calibrate VNA, S-Parameters, Radiation Pattern, and Positioner Controller. The following four sub-sections and Figures 14-17 present a detailed explanation of each tab and their features. The logic flowchart for the LabVIEW AMS can be seen in Appendix D. 


\subsection{Calibration}

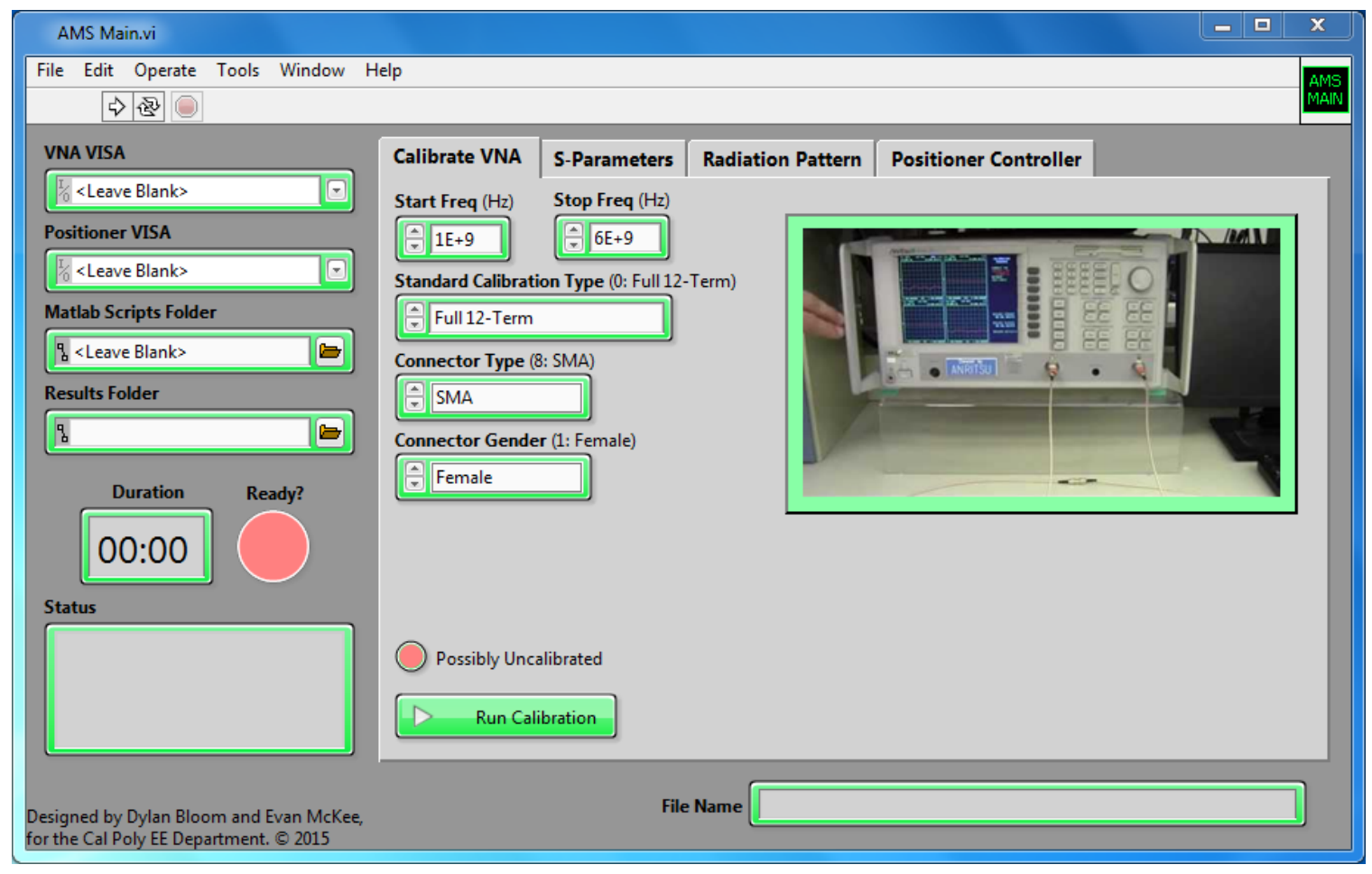

Figure 14 LabVIEW AMS Front Panel: Calibration

Remote calibration follows the same procedure as manual calibration except the settings are all located on one computer screen. Numeric controls specify the start and stop frequency, while menu controls specify the calibration type, connector type, and connector gender. LabVIEW compiles these settings into an SCPI command sequence and a "Run Calibration" button sends the command sequence to the VNA. For each calibration standard (open, short, broadband, through line) a Measure Device dialog box pops up in LabVIEW. The VNA indicates which calibration standard(s) to connect, and clicking "OK" in the dialog box triggers the VNA to measure the device(s).

Calibration accounts for losses in the coaxial cables and air between the antennas for accurate transmission measurements. During full 12-term calibration, point the AUT at boresight with the standard gain antenna, and use this as the through line 
reference. This ensures that S21 measurements will be normalized to the main beam strength. Also, note the user must manually calibrate the HP network analyzer because it is already simple to do manually. When the AMS detects the HP network analyzer, it disables the calibrate VNA tab.

\subsection{Measuring S-Parameters}

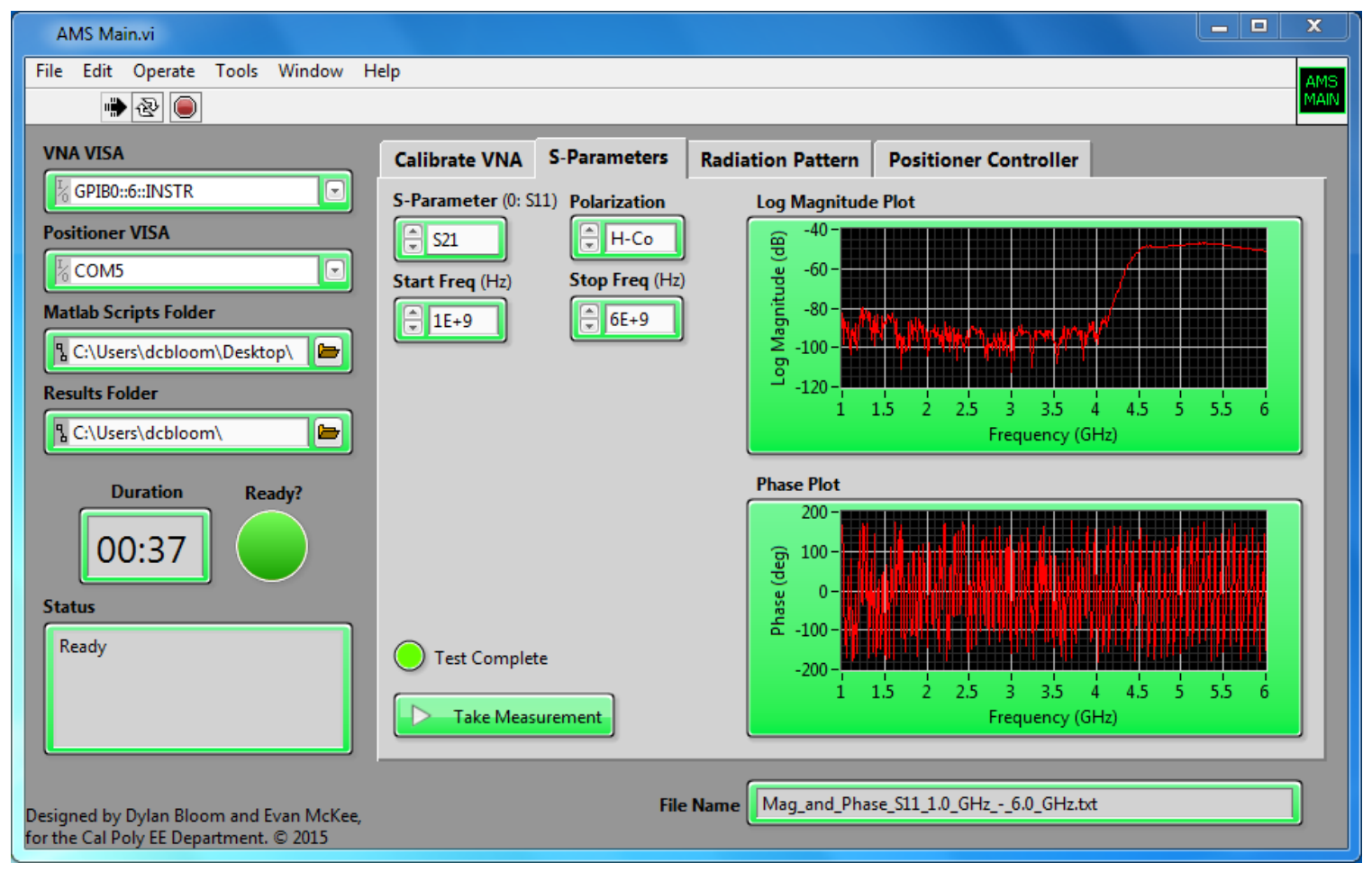

Figure 15 LabVIEW AMS Front Panel: S-parameter Measurement

S-parameter measurements form the foundation of the antenna measurement system. Numeric controls in the S-Parameters tab specify which S-parameter to measure, as well as the start and stop frequency, and polarization when applicable. If S11 or S22 are selected, LabVIEW disables the polarization control. Disabling certain controls not necessary for specific modes helps users focus only on relevant settings. Software prediction and ease of use features such as this are discussed in section 3.5. 
LabVIEW calls the VNA - Measure S-Parameter sub VI which configures the channel layout, active channel, channel graph type, sweep range, starts the sweep, and then reads channel data from the connected VNA. Next, the main VI adds a header to the data and saves it as a tab-delimited text file to a user specified results folder. LabVIEW generates the file name from the test settings and displays it below the graphs on the main front panel. Underscores instead of spaces to make the file name more compatible with post-processing programs. Plots on the front panel display log magnitude in $\mathrm{dB}$ as well as signal phase.

\subsection{Radiation Pattern Sweep}

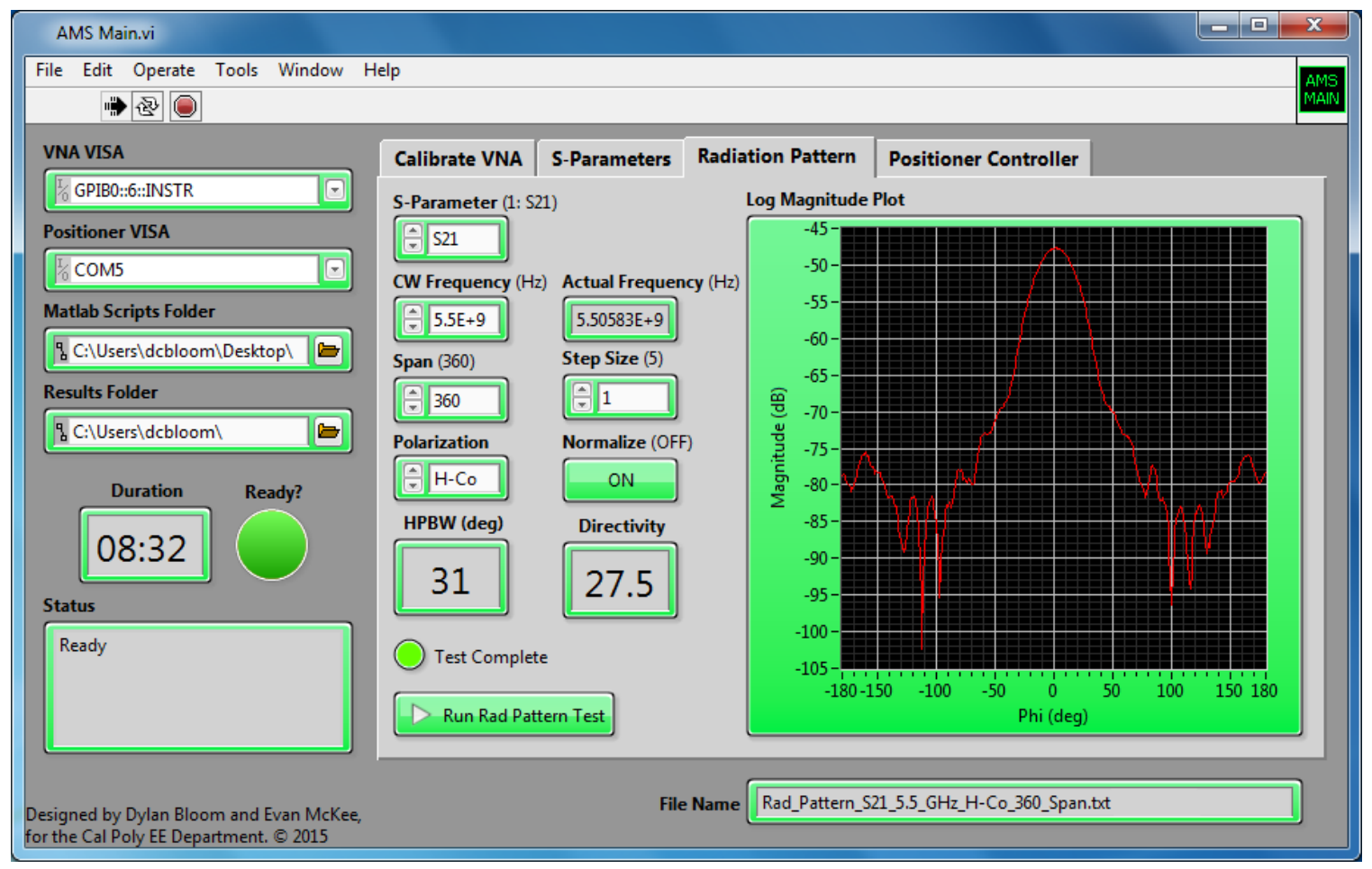

Figure 16 LabVIEW AMS Front Panel: Radiation Pattern (uncalibrated)

A radiation pattern sweep is composed of repeated transmission measurements synchronized with AUT rotation. This is the heart of the test software. The system takes 
an averaged S21 measurement for each step along the $360^{\circ}$ rotation of the AUT. The user specifies the step size as well as the total sweep span in degrees, but the default is $1^{\circ}$ steps over a $360^{\circ}$ span. The system starts by moving the AUT to the $-180^{\circ}$ position, facing away from the receive antenna, then steps clockwise as seen from above through $0^{\circ}$ and finishing at $+180^{\circ}$. After the sweep, the positioner returns counterclockwise to $0^{\circ}$ and the test results are saved to a text file. If the user specifies a sweep span smaller than $360^{\circ}$, such as $90^{\circ}$, the system will sweep from - Span/2 to + Span/2, e.g. $-45^{\circ}$ to $+45^{\circ}$. Measurements are always centered about the $0^{\circ}$ position, the direction facing the receive antenna. A reduced sweep saves time when taking multiple measurements where only the main beam of the antenna is important. In cases where the antenna pattern is unknown, users should perform a full $360^{\circ}$ sweep.

The system displays an S21 Magnitude plot as it records each data point so users can verify that measurements are as expected as the test progresses. As soon as the user clicks the start button, labeled "Run Rad Pattern Test", it becomes a "Cancel Sweep" button. The "Cancel Sweep" button throws out any collected data and returns the positioner to $0^{\circ}$, completely reinitializing the test. It is also possible to export any plot in LabVIEW directly from the front panel by right clicking and selecting "export data to Excel." This is a useful feature for examining specific data points.

The AMS only allows radiation pattern sweeps in constant wavelength (CW) mode. This cuts down on system complexity and sweep time. Shorter sweep times allow more time for designing systems instead of testing them. When the user inputs a measurement frequency, LabVIEW returns the nearest calibrated frequency point. Next, the user must specify the polarization of the antennas as H-Plane or E-Plane and copolarized or cross polarized. The actual frequency and the polarization mode are written into the name of the file when LabVIEW saves the data. 
Once a sweep completes, LabVIEW saves the S21 vs Phi Angle data in tabular format and post processing can begin. LabVIEW calls one Matlab script to calculate HPBW and directivity, and another to graph the radiation pattern on a polar plot. These Matlab scripts are described in section 3.6.

To only measure relative power, a "Normalize" button tells Matlab to subtract the peak value from the rest of the measurements. This sets the plot axis min to $0 \mathrm{~dB}$ and max to -infinity with respect to the main radiation direction. This is particularly useful when the antenna pattern is unknown beforehand. A fully calibrated measurement can be run afterward. 


\subsection{Positioner Controller}

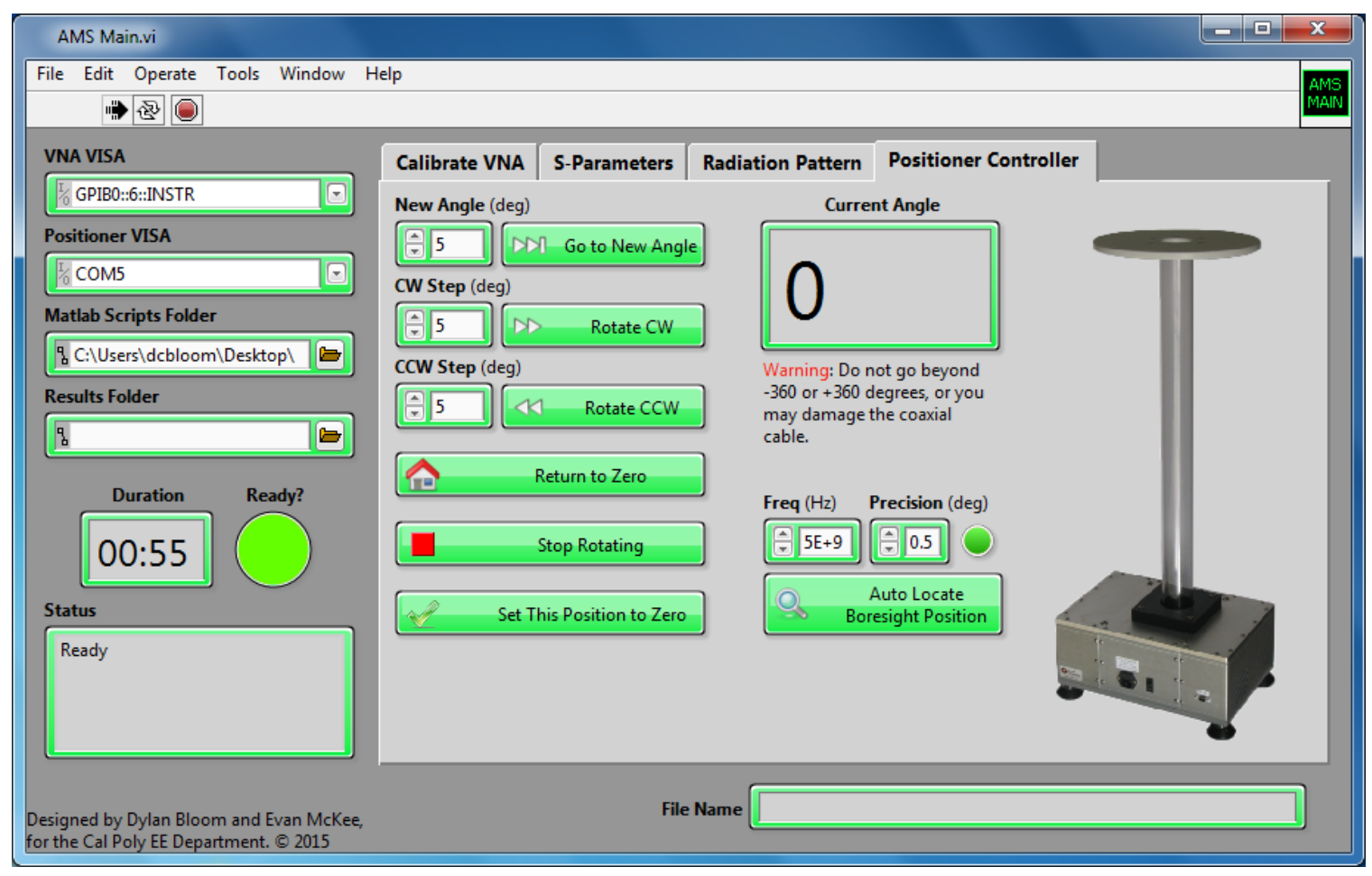

Figure 17 LabVIEW AMS Front Panel: Positioner Controller

The Positioner Controller tab helps set up the start location of the FS-121 positioner. It has all the controls necessary to orient the AUT before scanning. UI buttons step the positioner clockwise or counter clockwise, as seen from above, and another button zeroes out the positioner once it has reached the desired start location. A large numeric indicator displays the positioner's current angle. Although the positioner can rotate indefinitely, steps are limited to a $\max$ of $360^{\circ}$. There is also a "Stop Rotating" button to prevent the positioner from over rotating and damaging any cables. The numerical step size controls are limited to prohibit large or negative numbers that would otherwise damage the equipment. Even with these prevention features, the user must take care not to over rotate the positioner. Also, note that when the positioner is first powered on or initialized, it reports its location as $0^{\circ}$. 


\subsubsection{Auto Locate Boresight}

One special feature of the Positioner Controller tab is the "Auto Locate Boresight Position" button. This button calls a sub VI that steps the positioner until it locates the strongest transmission point between the two antennas for a given frequency. It assumes the antennas are properly mounted and connected to the VNA, then uses a basic peak search algorithm. The system steps the AUT in fractional degree steps until it finds a peak in S21. The algorithm only locates the first maximum it comes across. It is not sufficiently intelligent to ignore pattern lobes other than the main beam, so one must point the AUT in the general direction of the receive antenna first. The default step size is $0.5^{\circ}$, but is user adjustable. Greater step sizes can be less accurate because they could overlook the main peak. On the other hand, finer step sizes can be less accurate because small peaks can be mistaken for the main peak.

The auto locate function is most useful when one knows the antenna's general pattern before testing. Once the algorithm locates a peak, an indicator next to the button turns green and the positioner is reinitialized to $0^{\circ}$. The auto locate feature can make aligning the AUT a single click process. 


\subsection{UI Features and Smart Controls}

The AMS VI has intelligent indicators and prediction features that make it fast and friendly for new users.

\subsubsection{Status Indicators}

A status cluster in the bottom left corner of the main VI conveys all vital operating information. It includes the elapsed time, a main indicator light, and a general text display. The main indicator light starts off red, turns yellow during a test or initialization, and turns green once the system is ready for a test. The general text display provides more information such as which instrument LabVIEW is initializing, whether the program has encountered an error, what that error is, and which test the system is running. During the radiation pattern test, the status box also displays the last azimuth angle measured.

Each tab also features its own indicator light and text display to provide yet more information. For example, the Calibrate VNA tab displays a red light and the text "Possibly Uncalibrated" when the calibration procedure has not yet run. It displays a yellow light and the text "Calibrating" while a calibration is in progress. Intelligent status indicators keep the user informed, increasing their comprehension of each test.

\subsubsection{Intelligent Initialization}

To communicate with external instruments, LabVIEW needs to know the hardware addresses and limitations of those instruments. Normally the user must manually enter the instrument VISA addresses, but this can be confusing for users who

do not need to understand this underlying process. Having a fixed VISA address will not 
work all the time either because GPIB and USB addresses can change. Instead, the AMS intelligently identifies and initializes any properly connected equipment.

After clicking the run arrow, the main VI searches for the positioner and any known VNA connected to the computer. The VNA - Find VISA sub VI first identifies all active GPIB VISA addresses to narrow the search. Then it calls the VNA - Get Mode/ VI to query each instrument's manufacturer until it finds Anritsu or Hewlett Packard. The Positioner - Find VISA sub VI builds an array of all possible COM ports, or serial ports (COM1, COM2, COM3, etc.) and loops until it recognizes the positioner and returns its unique address. As a check, if the VNA or positioner VISA addresses are still empty, a window pops up telling the user to select an instrument. Also at this point, AMS Main initializes all front panel indicators depending on the identified VNA model. For example, if the system recognizes the Anritsu MS4622B (see Table 2), it will limit user input for any frequency controls to between $10 \mathrm{MHz}$ and $3 \mathrm{GHz}$. This protects the system from users entering frequencies that instrument is incapable of.

Similarly, for LabVIEW to access the Matlab post-processing scripts, it needs a pointer to the directory where they are located. Because the LabVIEW executable can be installed in any directory on any machine, this pointer needs to be dynamic to change with each install. When a user downloads the AMS.zip file, the scripts are included in the Matlab Scripts folder. LabVIEW automatically finds this folder using a built-in function to acquire its current working directory and append the known file names to that path. For this reason, it is important to maintain the folder hierarchy of the download.

Finally, LabVIEW attempts to initialize the VNA and Positioner. Initializing the VNA consists of a software reset to return the instrument to a known state. Initializing the antenna positioner consists of clearing its communication buffer. If either initialization fails, a pop up window tells the user to check connections and power cycle the 
malfunctioning instrument. This self-diagnostic feature saves time by pointing to a specific instrument and giving specific instructions to remedy it.

\subsection{Matlab}

After a radiation pattern sweep, LabVIEW saves the S21 Magnitude and azimuth angle data to a tabular file. LabVIEW then calls two Matlab functions in series to graph the data on a polar plot and calculate the pattern directivity and HPBW. While LabVIEW can perform these operations within its native environment, its graphical data flow style is more suited to data collection rather than data processing. Matlab is highly capable at processing large amounts of data, and a few simple lines of code can replace a nest of wires and blocks in LabVIEW. Also, one can call these Matlab functions independently of the LabVIEW environment to reprocess already collected or modified data files. Matlab source code can be found in Appendices F and G.

\subsubsection{Radiation Pattern Plot}

RadPatternPlot.m calls dirplot.m to graph radiation pattern data from a file recorded with the Cal Poly LabVIEW AMS. dirplot.m is an open source Matlab function available online [23] that modifies Matlab's "polar" function to graph data on a negative raxis. Inputs to RadPatternPlot.m include the data file, plot style, and the option to "normalize" as described in section 3.3. A radiation pattern data file should be three columns: angle, magnitude, and phase, ordered from $-180^{\circ}$ to $+180^{\circ}$. Radiation pattern plots appear with $0^{\circ}$ in the North direction and represent the antenna pattern as viewed from above the test fixture. RadPatternPlot.m does not return any values, and the user must manually save the plots it generates. 


\subsubsection{Directivity}

Directivity.m calculates HPBW and directivity from radiation pattern data. HPBW gets extrapolated into a solid beam angle then used to estimate directivity. The function expects data in the same format as RadPatternPlot.m, from $180^{\circ}$ to $+180^{\circ}$. Directivity.m first finds the peak magnitude of the data, then it finds the first point on either side of the peak where the magnitude drops $3.01 \mathrm{~dB}$ below the maximum. Generally, a smaller step size produces more accurate results.

While a smaller step size provides more data points to analyze, it makes the test take longer to complete. To reduce test time Directivity.m and RadPatternPlot.m accept partial patterns (e.g. $-90^{\circ}$ to $+90^{\circ}$ ) as long as they include half power points on either side of the main beam. Calculations for solid beam angle and directivity can be seen in the Matlab script in Appendix G. 
CHAPTER 4: Results and Verification

The goal of this project is to produce an antenna measurement system that meets or exceeds the performance of Cal Poly's existing 20' anechoic chamber. To verify the new anechoic chamber and its LabVIEW code, results from it must be compared against results taken from identical measurements made in the existing chamber. This chapter compares data from four different pairs of antennas measured in the same style in both the Microwave Lab's new chamber and the Hangar Lab's existing chamber. The results are referred to as "Microwave Lab" data or "Hangar Lab" data. The method for measurement is a $360^{\circ}$ rotation sweep with measurements at $1^{\circ}$ steps. Both chambers are evaluated on their representation of each antenna's radiation pattern when compared to an ideal antenna mathematical model. If the Microwave Lab data fits the mathematical model as well as or better than the Hangar Lab data, the new AMS will be considered a success.

Furthermore, the chamber which produces radiation patterns for each antenna closest to their respective mathematical model will be declared most accurate, notwithstanding unforeseen measurement errors. The simple mathematical model for an antenna takes into account the length of the main transmitting surfaces of the antenna and predicts how the radiation from those sources will combine in the far field to create nodes and nulls. The chambers can be evaluated by their detection of the predicted nodes and nulls. This model is discussed further in section 4.2.

One last consideration when undertaking this comparison is the fact that the Microwave Lab chamber is about half the size of the Hangar Lab chamber. One can anticipate the differences between two antenna measurement systems that are similar in every way except for the size of their anechoic chambers. A larger chamber must have 
some sort of advantage over a smaller chamber, otherwise there would be no need for such large, expensive chambers. After all, the goal of using an anechoic chamber is to simulate a large open space.

Other than the obvious answer that the larger chamber can fit larger antennas, larger chambers also have the advantage of greater antenna separation distance. A greater distance between the antenna under test and the probe detecting its radiation allows for more wavelengths between the two, to better measure the far field radiation pattern. This means that lower frequency antennas with longer wavelengths are better accommodated by larger chambers that can put more wavelengths between the antenna and the probe. However, there is a trade off. Greater separation distance means that less power gets delivered to the probe, as the probe takes up a smaller arc area of the AUT's radiation at that distance. The result is an $\$ 21$ signal that gets buried in the noise floor of the system, and greater power via amplification is needed going into the AUT to bring the signal above the noise. An amplifier adds parts, cost, and complexity, but nonetheless that is the trade off. Below is a table of the expected differences between large and small anechoic chambers.

Table 3 Differences Between Small and Large Anechoic Chambers

\begin{tabular}{|l|l|}
\multicolumn{1}{|c|}{ Smaller Chamber } & \multicolumn{1}{c|}{ Bigger Chamber } \\
\hline Less expensive & More expensive \\
\hline $\begin{array}{l}\text { Can only measure smaller } \\
\text { antennas }\end{array}$ & $\begin{array}{l}\text { Can also measure physically } \\
\text { bigger antennas }\end{array}$ \\
\hline $\begin{array}{l}\text { Can only measure antennas at } \\
\text { higher frequencies }\end{array}$ & $\begin{array}{l}\text { Can also measure antennas at } \\
\text { lower frequencies }\end{array}$ \\
\hline $\begin{array}{l}\text { Usually does not require an } \\
\text { amplifier to maintain SNR }\end{array}$ & $\begin{array}{l}\text { May require an amplifier to } \\
\text { maintain SNR }\end{array}$ \\
\hline
\end{tabular}




\subsection{Choosing the Antennas}

There are four constraints to qualify antennas for use in this comparison test. First, each antenna must come in a matching pair. Although it is not necessary to use the same antenna to receive a signal as was used to transmit that signal, using the same antenna is one way to be sure that the transmission can be detected if a broadband RF detector is not available. In this case, using a pair of identical antennas also offers more flexibility in choosing which frequency to transmit over choosing two different antennas because both antennas have the same operating frequency range. What one antenna transmits, the other antenna is guaranteed to be able to receive, so long as they are in the co-polarized configuration.

Second, each antenna must operate partially within the VNA limit. Even though the VNA in the Hangar Lab has an upper frequency limit of $20 \mathrm{GHz}$, antennas are limited to the lowest common maximum frequency between the VNAs in each lab. This is because the two labs are too far to move the sensitive and expensive $20 \mathrm{GHz}$ VNA between them. The VNA in Microwave Lab has an upper frequency limit of $6 \mathrm{GHz}$, and this is the limit for this test.

Third, the antennas must be small enough to fit inside the Microwave Lab

Chamber because it is the smaller of the two chambers. The Microwave Lab Chamber can only accommodate an antenna up to $0.6 \mathrm{~m}$ in any dimension because of the size of the openings and load capability of the robotic positioner.

Finally, the fourth constraint is due to the length of the Microwave Lab anechoic chamber. The distance between the two antennas must be long enough so the antennas are in the far field. Although frequency alone does not dictate the far field distance, it is closely related. Because lower frequencies have a longer wavelength, the max possible separation distance decides the minimum frequency. Far field is also a factor of 
aperture, or the largest transmitting surface of the antenna. Larger antennas have longer far field distances, requiring them to being measured in larger chambers. Far field calculations are available in Appendix A. As a result, only antennas with a far field distance less than or about equal to $1 \mathrm{~m}$ are allowed. Table 3 shows the selected antennas and the frequencies at which they were tested, as well as their far field distance.

Table 4 Antennas for Chamber Verification

\begin{tabular}{|l|c|c|c|}
\hline \multicolumn{1}{|c|}{ Antenna } & $\begin{array}{c}\text { Operating } \\
\text { Range }\end{array}$ & $\begin{array}{c}\text { Frequency } \\
\text { Tested }\end{array}$ & $\begin{array}{l}\text { Far Field } \\
\text { Distance }\end{array}$ \\
\hline $\begin{array}{l}\text { Narda 642 } \\
\text { Standard } \\
\text { Gain Horn }\end{array}$ & $5.40-8.2 \mathrm{GHz}$ & $5.5 \mathrm{GHz}$ & $46.86 \mathrm{~cm}$ \\
\hline $\begin{array}{l}\text { Narda 643 } \\
\text { Standard } \\
\text { Gain Horn }\end{array}$ & $3.95-5.9 \mathrm{GHz}$ & $5.0 \mathrm{GHz}$ & $79.05 \mathrm{~cm}$ \\
\hline $\begin{array}{l}\text { 915 MHz } \\
\text { Patch }\end{array}$ & $\begin{array}{c}915 \mathrm{MHz} \\
\text { (narrowband) }\end{array}$ & $915 \mathrm{MHz}$ & $12.82 \mathrm{~cm}$ \\
\hline $\begin{array}{l}\text { Broadband } \\
\text { Horn }\end{array}$ & $0.7-18 \mathrm{GHz}$ & $3.0 \mathrm{GHz}$ & $101.25 \mathrm{~cm}$ \\
\hline
\end{tabular}




\subsection{Mathematical Model for Nodes and Nulls}

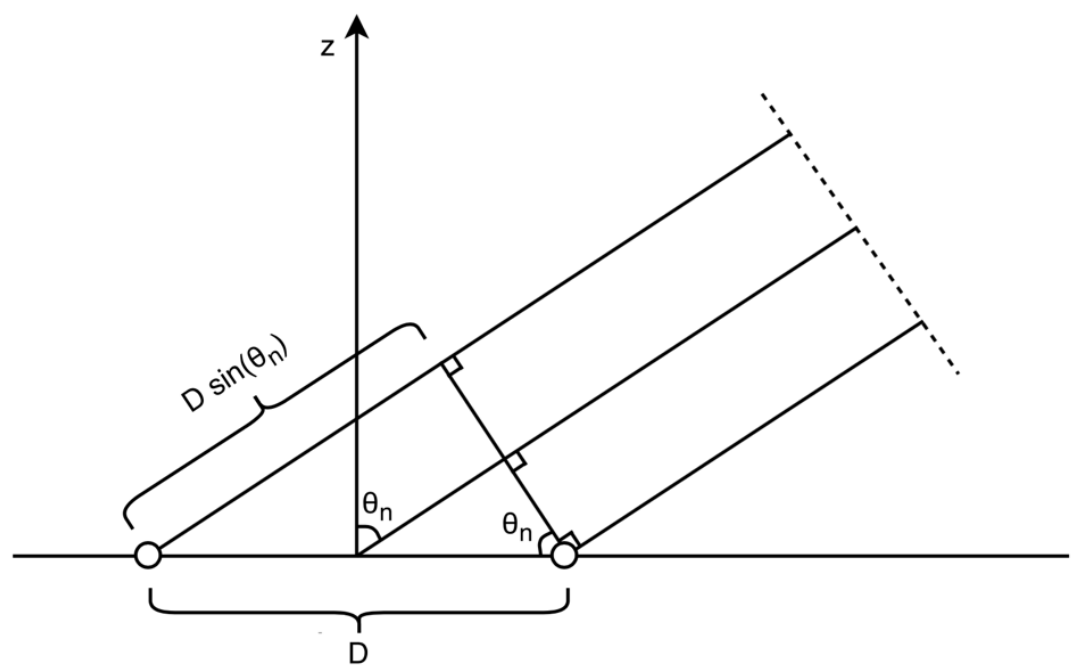

Figure 18 Far Field Parallel Ray Approximation

One can estimate the far field patter of an antenna by treating the points on its surface as independent, phase-locked sources. Calculating how signals from these sources combine in the far field identifies nodes where total emission is strongest, and nulls where it is weakest. Consider a radiating horn antenna. Electromagnetic waves emanate from either side of the aperture where they are initially $180^{\circ}$ out of phase. When the difference in distance traveled by the two signals to reach a single point is an integer multiple of one wavelength, their power will cancel out creating a pattern null. Correspondingly, when that difference is an odd integer multiple of a half wavelength, their power will combine to create a pattern node. Using the far field parallel ray approximation illustrated in Figure 18, this difference is calculated as $D \sin \left(\theta_{n}\right)$ where $D$ is the distance separating the two sources, and $\theta_{\mathrm{n}}$ is the angle of the parallel emissions away from the normal vector of the antenna. Setting this equal to $n^{\star} \lambda$ and $n^{\star} \lambda / 2$ and solving for $\theta_{\mathrm{n}}$ yields the following equations for the antenna pattern's node and null angles. 


$$
\begin{array}{ll}
\theta_{\text {null }}=\sin ^{-1}\left(\frac{n \lambda}{D}\right), \text { where } n=1,2,3, \ldots & \text { Equation } 6 \\
\theta_{\text {node }}=\sin ^{-1}\left(\frac{n \lambda}{2 D}\right), \text { where } n=1,3,5, \ldots & \text { Equation } 7
\end{array}
$$

The hand calculation method is useful for verifying patterns recorded by an AMS. For more detailed pattern analysis, 3D computer modeling tools such as Keysight's Advanced Design System (ADS) and Ansys' High Frequency Structural Simulator (HFSS) use finite element analysis to produce simulated field patterns. Table 4 shows the calculated far zone and the first two nodes and nulls of the antennas available in the Microwave Lab.

Table 5 Antenna Far Field Distances and Nulls

\begin{tabular}{|l|l|l|l|l|l|l|l|l|}
\hline $\begin{array}{l}\text { Antenna } \\
\text { Model }\end{array}$ & Frequency & $\begin{array}{l}\text { Aper- } \\
\text { ture }\end{array}$ & \multicolumn{1}{|c|}{$\begin{array}{l}\text { (cm) } \\
\text { (cm) }\end{array}$} & $\begin{array}{l}\text { Far } \\
\text { Zone } \\
\text { (cm) }\end{array}$ & $\begin{array}{l}\text { First } \\
\text { Null }\end{array}$ & $\begin{array}{l}\text { Second } \\
\text { Null }\end{array}$ & $\begin{array}{l}\text { First } \\
\text { Node }\end{array}$ & $\begin{array}{l}\text { Second } \\
\text { Node }\end{array}$ \\
\hline $\begin{array}{l}\text { Narda } \\
\mathbf{6 4 2}\end{array}$ & $5.5 \mathrm{GHz}$ & 11.3 & 5.45 & 46.86 & $28.8^{\circ}$ & $74.7^{\circ}$ & $13.9^{\circ}$ & $46.3^{\circ}$ \\
\hline $\begin{array}{l}\text { Narda } \\
\mathbf{6 4 3}\end{array}$ & $5.0 \mathrm{GHz}$ & 15.4 & 6.00 & 79.05 & $22.9^{\circ}$ & $51.2^{\circ}$ & $11.2^{\circ}$ & $35.8^{\circ}$ \\
\hline $\begin{array}{l}\text { Narda } \\
\mathbf{6 4 4}\end{array}$ & $2.4 \mathrm{GHz}$ & 23.1 & 12.50 & 85.38 & $32.8^{\circ}$ & - & $15.7^{\circ}$ & $54.3^{\circ}$ \\
\hline $\begin{array}{l}\text { BB } \\
\text { Horn }\end{array}$ & $3.0 \mathrm{GHz}$ & 22.5 & 10.00 & 101.25 & $26.4^{\circ}$ & $62.7^{\circ}$ & $12.8^{\circ}$ & $41.8^{\circ}$ \\
\hline $\begin{array}{l}\mathbf{9 1 5} \mathbf{M H z} \\
\text { Patch }\end{array}$ & $915 \mathrm{MHz}$ & 14.5 & 32.79 & 12.82 & - & - & - & - \\
\hline $\begin{array}{l}\mathbf{2 . 4} \mathbf{G H z} \\
\text { Horn }\end{array}$ & $2.4 \mathrm{GHz}$ & 24 & 12.50 & 92.16 & $31.4^{\circ}$ & - & $15.1^{\circ}$ & $51.4^{\circ}$ \\
\hline
\end{tabular}

\subsection{Radiation Patterns Graphed on Polar Plots}

First consider the polar plots of the antenna radiation pattern data. Polar plots, compared to $\mathrm{XY}$ plots, are good visualizing how the antenna actually transmits, and they 
are good for highlighting nodes and nulls. The Microwave Lab system automatically generates polar plots via Matlab, but the Hangar Lab system only generates a tabular data file. To ensure a fair comparison, polar plots of the data from the Hangar Lab were generated using the same Matlab script as the Microwave Lab Data. In the following plots Microwave Lab data is blue and Hangar Lab data is red. Data is presented in dB and both traces are on the same scale.

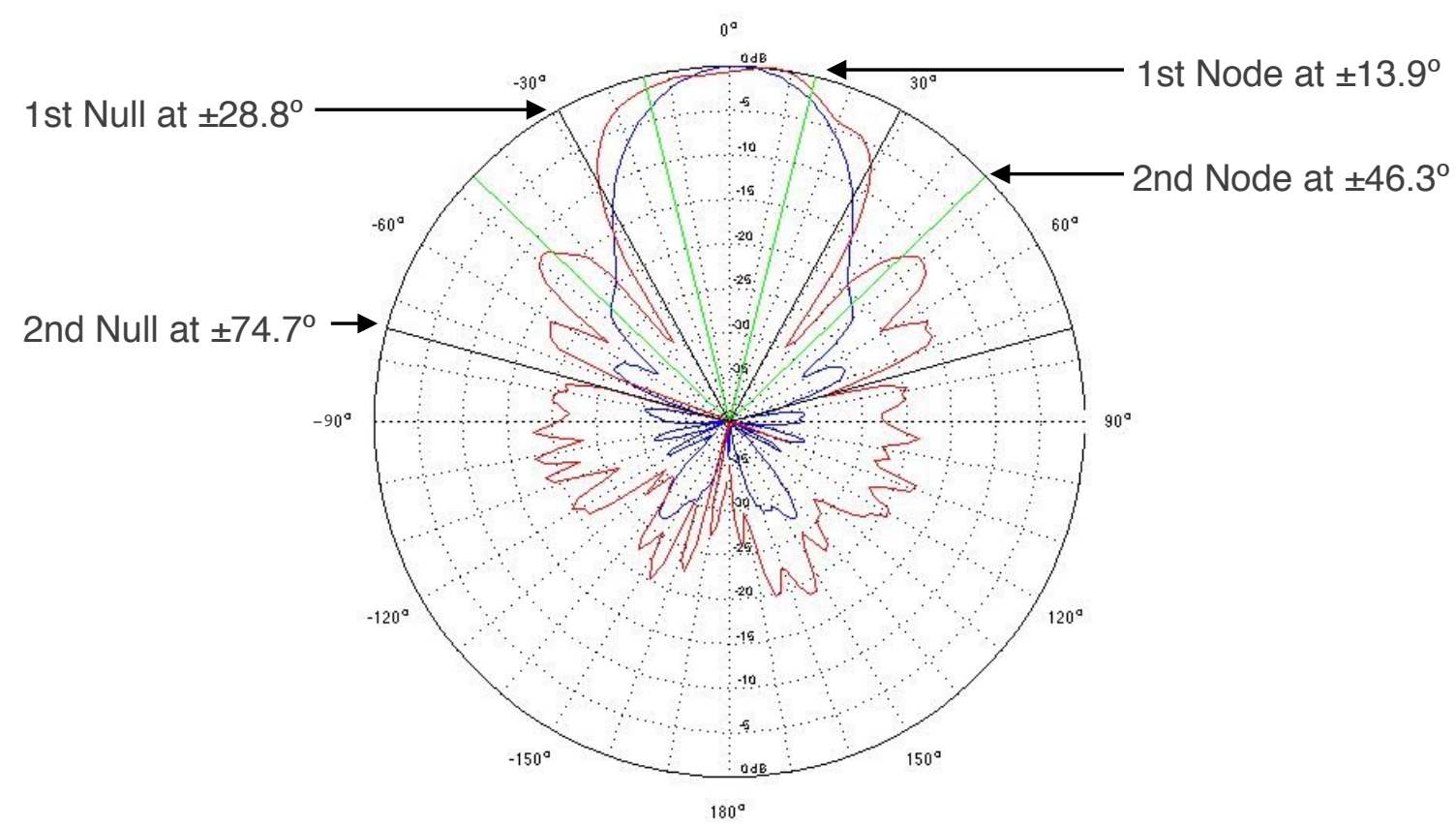

Figure 19 Polar Radiation Pattern Plot of the Narda 642 Horn Antenna at $5.5 \mathrm{GHz}$ as Measured by the Microwave Lab Chamber (Blue) and Hangar Lab Chamber (Red), and Predicted 1st and 2nd Nodes (Green) and Nulls (Black)

In Figure 19, both chambers accurately measure the main beam of the the Narda 642 Horn antenna. The predicted nodes at $\pm 13.95^{\circ}$ lie on top of the main lobe, and this is as expected because the main lobe is technically the first node. Next, the first predicted null does not line up perfectly with a measured null from either AMS, however both patterns experience a drop in $\mathrm{S} 21$ at $37^{\circ}$, within $10^{\circ}$ of the estimated location. The null is 
more apparent in the Hangar Lab AMS with a drop of $-20 \mathrm{~dB}$ at the null versus the surrounding pattern. Meanwhile the Microwave Lab pattern shows less of a distinct notch at this angle but more of a gradual decline until the subsequent node angle. The second nodes and second nulls line up with both antenna patterns, yet they are again more apparent in the Hangar Lab measurement than in the Microwave Lab measurement.

One possible explanation for this null magnitude difference between the two measurement systems, ignoring the additional noise in the Hangar Lab data, is that the Hangar Lab is larger and puts more distance between the two antennas. A greater distance between the antennas gives their radiation more space to interfere with itself, thus accentuating the nodes and nulls.

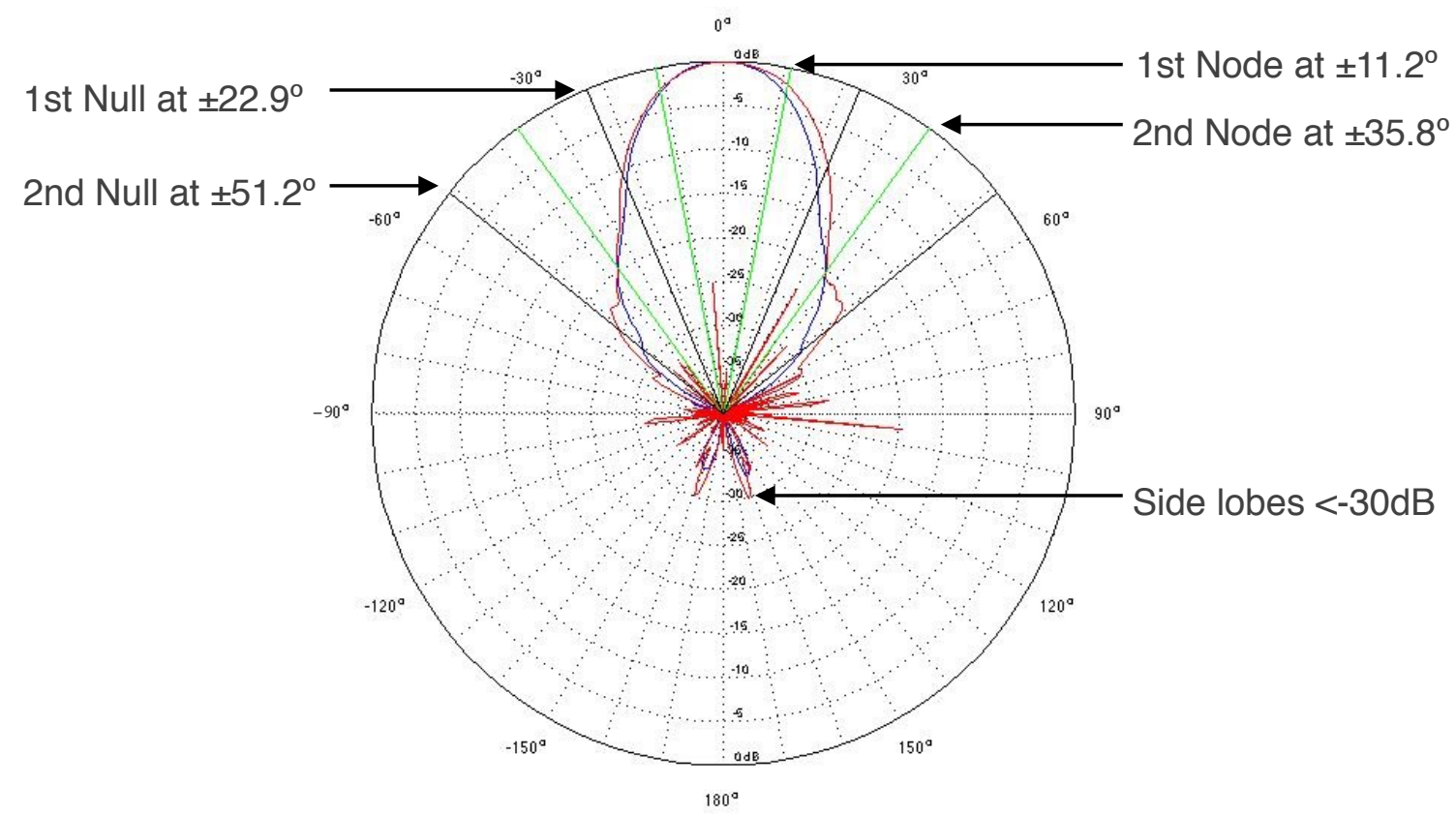

Figure 20 Polar Radiation Pattern Plot of the Narda 643 Horn Antenna at $5.0 \mathrm{GHz}$ as Measured by the Microwave Lab Chamber (Blue) and Hangar Lab Chamber (Red), and Predicted 1st and 2nd Nodes (Green) and Nulls (Black) 
Moving on to the second antenna, the Narda 643 horn, the pattern has a more focused main beam at $5.0 \mathrm{GHz}$ compared to the Narda 642 horn at $5.5 \mathrm{GHz}$, with a HPBW of nearly $10^{\circ}$ versus $15^{\circ}$ of the 642 . The 643 Horn also has fewer to no side lobes outside of $\pm 60^{\circ}$ above the $-30 \mathrm{~dB}$ level. Both measurement systems accurately detect the predicted nulls and nodes. Again, the first nodes lie in the main beam. The first nulls contain $10 \mathrm{~dB}$ less power than the $0^{\circ}$ direction. The second nodes are given away by a decrease in the negative slope in each of the measurements at $\pm 35^{\circ}$. The second nulls lie around $30 \mathrm{~dB}$ below the $0^{\circ}$ direction. Despite the accuracy of the Hangar Lab AMS in measuring nodes and nulls, it records sharp discontinuities on the order of $10 \mathrm{~s}$ of $\mathrm{dB}$ inconsistent with the parallel ray approximation model. This noise hints that one or more of the model's assumptions are wrong in the Hangar Lab.

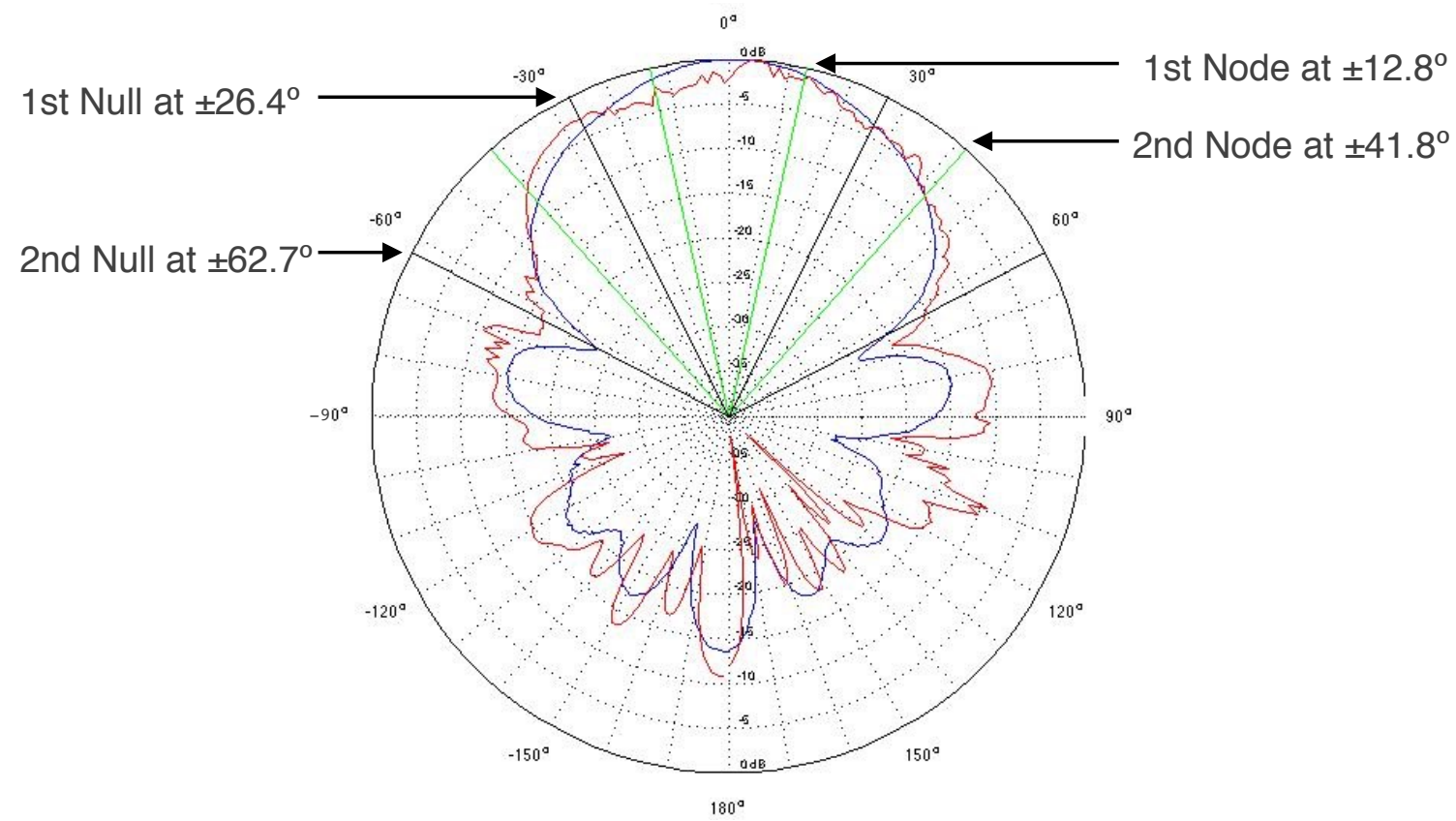

Figure 21 Polar Radiation Pattern Plot of the Broadband Horn Antenna at $3.0 \mathrm{GHz}$ as Measured by the Microwave Lab Chamber (Blue) and Hangar Lab Chamber (Red), and Predicted 1st and 2nd Nodes (Green) and Nulls (Black) 
Here, both chambers showed the broadband horn antenna is not as directive as either of the first two horn antennas, with a HPBW of $24^{\circ}$. This is as expected because in order to achieve good broadband performance, the antenna must give up some of its gain. Both chambers recorded a well defined second null at $\pm 62.7^{\circ}$ with $1^{\circ}-3^{\circ}$ margin of error. This error could be due to initial alignment and setup of the antenna under test. The first two nodes lie in the main beam, and the first null does not have much of an effect on the main beam. The reason this antenna is so broadband is because it is designed with many levels of emitting surfaces instead of a singular monolithic design like the previous two antennas. This makes its radiation pattern much more complex to predict and requires the use of computer modeling to calculate. Fortunately, it is just as easy to measure in the anechoic chamber. Figure 22 shows an example of the type of broadband horn used in this test. The interior ridges and bars lining the exterior work to enable multiple resonating modes for different sections of its operating frequency range.

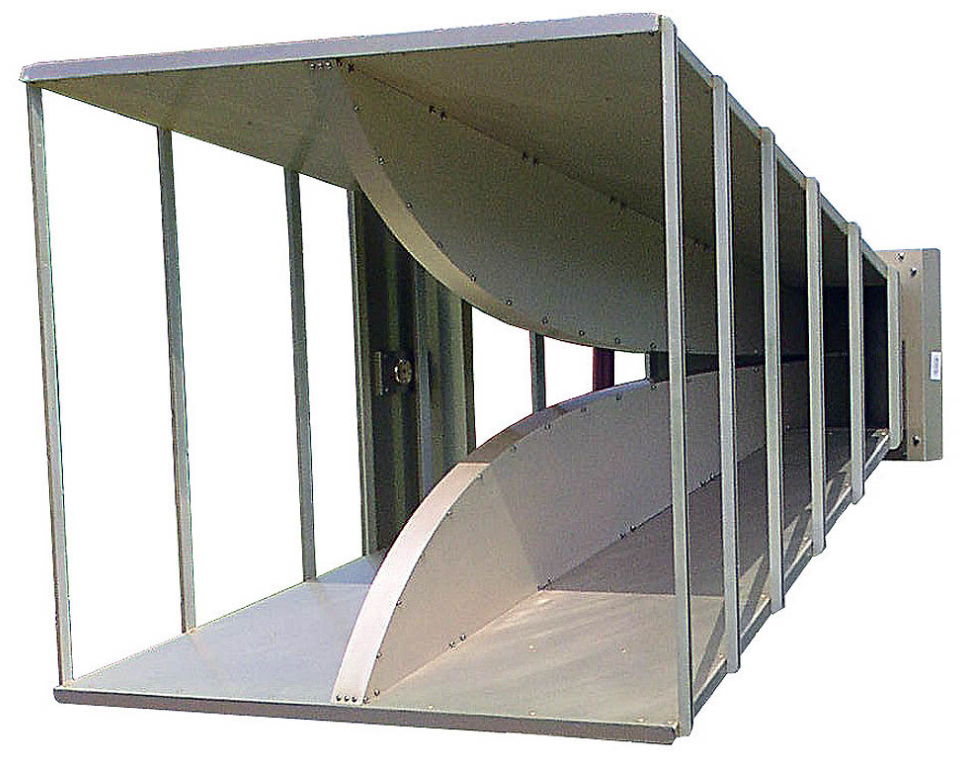

Figure 22 A Double Ridged Broadband Horn Antenna 


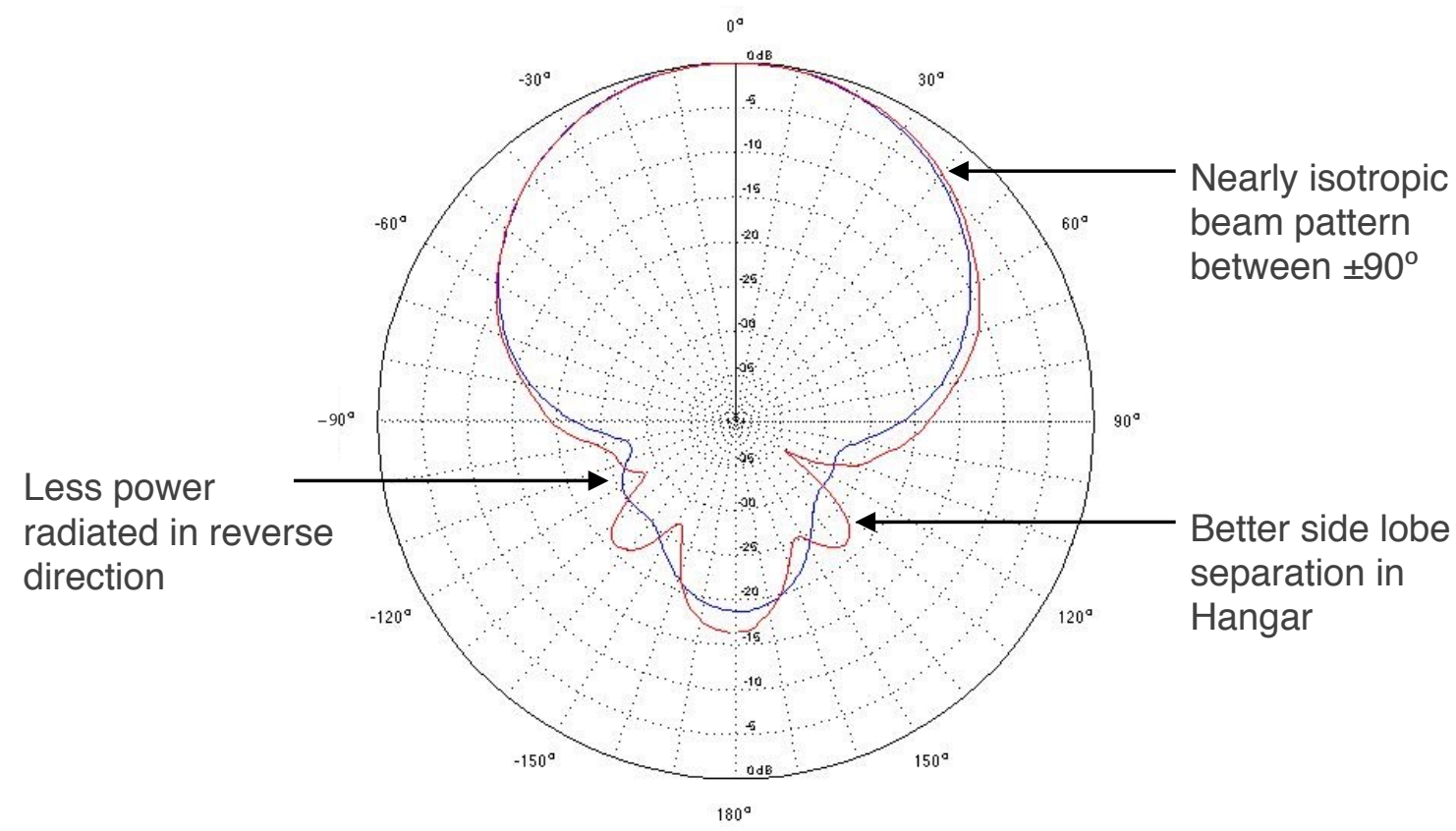

Figure 23 Polar Radiation Pattern Plot of the $915 \mathrm{MHz}$ Patch Antenna at $915 \mathrm{MHz}$ as Measured by the Microwave Lab Chamber (Blue) and Hangar Lab Chamber (Red)

The last antenna is a $915 \mathrm{MHz}$ patch antenna. Patch antennas are known for being very narrow band, and having a relatively isotropic beam pattern, but only in one direction. At $915 \mathrm{MHz}$, this antenna does not have any real valued nodes or nulls, however it is not expected to radiate well in the reverse direction. Both chambers confirm this expectation with smooth beam patterns until the $90^{\circ}$ mark. The Hangar Chamber records better separation of the reverse direction side lobes, whereas in the Microwave Lab Chamber these lobes are not as defined and blend together. This is consistent with the theory that a larger chamber will produce better separation of the side-lobes because of its greater antenna separation distance. 


\subsection{Realizations about Calibration Procedure}

Now examine data from just the Narda 642 horn graphed on an XY scatter plot to gain a different perspective. The XY plot can better show data from each chamber in the original S21 loss level as measured by the VNA, whereas the data before was normalized with respect to the main beam.

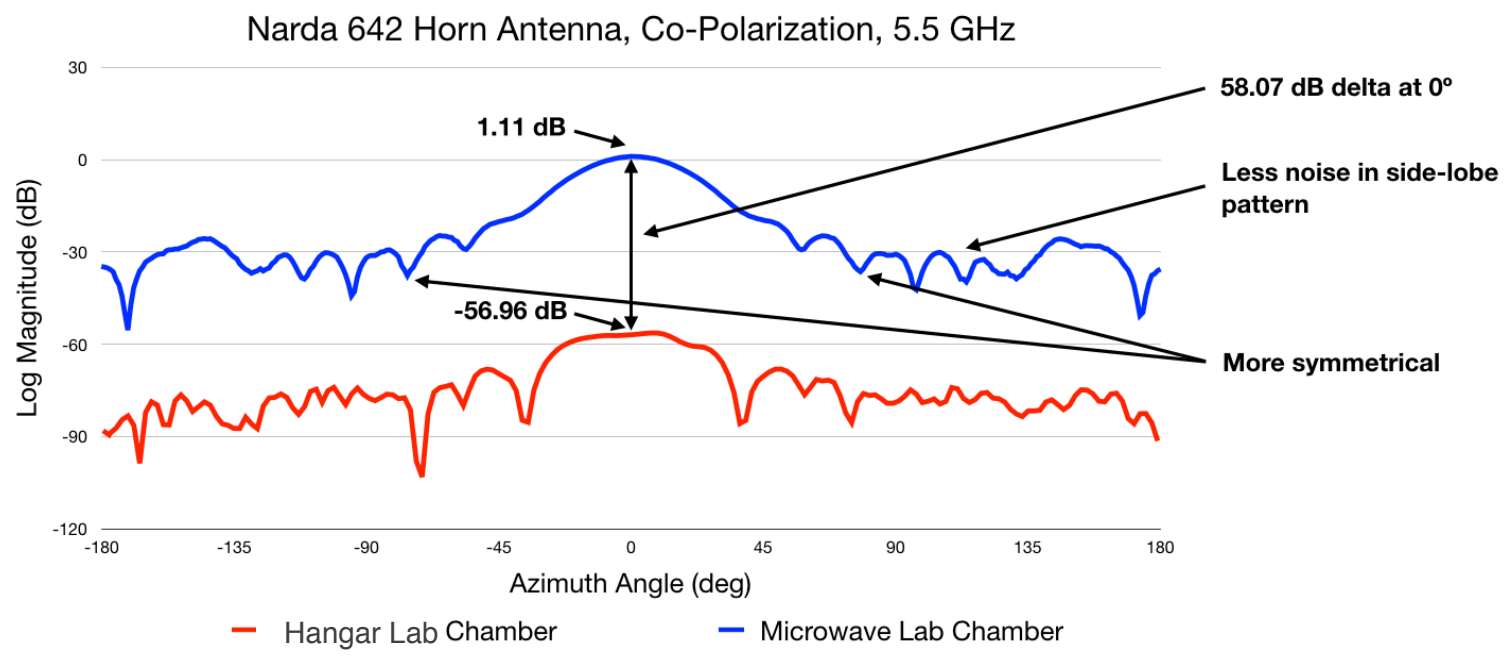

Figure 24 Power vs Azimuth Scatter Plot of the Narda 642 Horn Antenna at $5.5 \mathrm{GHz}$ as

Measured by the Microwave Lab Chamber (Blue) and Hangar Lab Chamber (Red)

Figure 24 shows an unusually large $57 \mathrm{~dB}$ difference in the transmission loss recorded by both chambers. Because the Hangar Lab chamber is larger and the antenna separation distance is greater, it is expected that the transmission loss there would be somewhat greater than that of the Microwave Lab chamber. However, after analyzing this data, it became apparent that parts of the measurement procedure for each chamber were incorrect. The difference in separation distance between the two chambers is not great enough to justify the $57 \mathrm{~dB}$ difference in main beam transmission loss. The separation distance in the Hangar Lab is about $2.5 x$ the separation distance in the Microwave Lab. Using the rule that radiative power decreases at a $r^{\wedge} 2$ rate from the 
source, we can estimate that the difference in log magnitude transmission loss between the two chambers for a given pair of antennas should be about $10^{\star} \log \left(2.5^{\wedge} 2\right)$ or $8 \mathrm{~dB}$.

The main discrepancy between the test procedures for each chamber was the calibration procedure and the VNA settings. The calibration procedures for each chamber previously were as follows.

Table 6 Original Calibration Procedure for Both Antenna Measurement Systems

\begin{tabular}{|c|c|c|}
\hline Calibration Step & Microwave Lab & Hangar Lab \\
\hline Broadband Load & $50 \mathrm{ohm}$ load at the end of each cable & Not measured \\
\hline Short & Short terminator at end of each cable & Not measured \\
\hline Open & Open terminator at end of each cable & Not measured \\
\hline Through Line & $\begin{array}{l}\text { Connect both antennas, point them at } \\
\text { each other, and measure }\end{array}$ & Not measured \\
\hline Isolation & Omitted & Not measured \\
\hline
\end{tabular}

Previously, in the Hangar Lab, it was assumed that the VNA need not be calibrated before measuring an antenna pattern. This is the way tests had always been run there. The theory was that because all the measurements taken during a radiation pattern scan are relative to each other, calibration was not necessary. However, this assumption was soon proven false, calibration does matter. This also explains why the Hangar Lab data was much noisier across all measured angles compared to the Microwave Lab data which had at least some form of calibration. The sharp discontinuities and asymmetries exhibited in the Hangar Lab data turned out to be a result of the missing calibration.

After calibrating the VNAs in both chambers in an identical fashion, the results from both chambers were much more similar to each other and were consistent with theory. Additionally, less noise, and more symmetrical patterns were observed. The new calibration sequence for both chambers, a full 12-term calibration, is as follows. 
Table 7 New Calibration Procedure for Both Antenna Measurement Systems

\begin{tabular}{|l|l|}
\hline Calibration Step & Microwave Lab and Hangar Lab \\
\hline Broadband Load & 50 ohm load at end of each cable \\
\hline Short & Short terminator at end of each cable \\
\hline Open & Open terminator at end of each cable \\
\hline Through Line & Connect the ends of both cables together \\
\hline Isolation & Omitted \\
\hline
\end{tabular}

New measurements were taken with the antennas that were still available using calibrated equipment. Additionally, noise floor measurements for each antenna were taken to show the accuracy of the weakest points of the measured patterns. A noise floor measurement is performed by connecting a $50 \mathrm{ohm}$ load to the end of the cable going to the antenna under test and then measuring S21 like normal. 
4.5 New Antenna Patterns with Calibrated Equipment

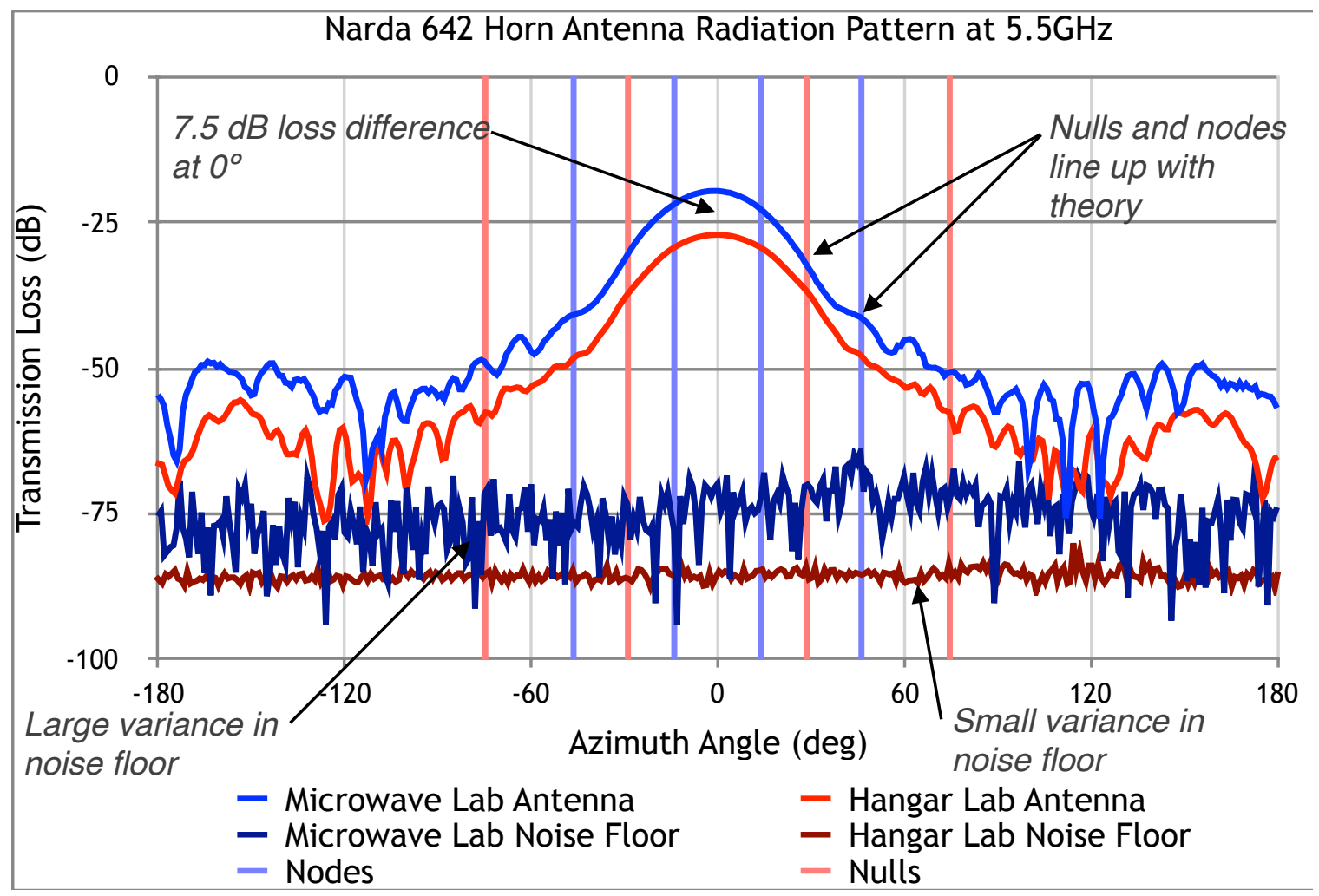

Figure 25 XY Scatter Plot of Narda 642 Horn Antenna Pattern Measured in Both Chambers with Noise Floor, Nodes and Nulls

First look at the Narda 642 antenna. With proper calibration, the difference in transmission loss between the two anechoic chambers becomes $7.5 \mathrm{~dB}$ at $0^{\circ}$ and $7.7 \mathrm{~dB}$ on average overall. This is line with our estimation of $8 \mathrm{~dB}$ in the previous section, reassuring that the data is now correct. We can also see that the nulls and nodes, particularly the first two nodes and the first nulls line up with recorded pattern from both chambers. The patterns overall are very similar, however the pattern from the Microwave Lab chamber appears less symmetrical and more noisy in the reverse facing side lobes beyond the $\pm 90^{\circ}$ mark in either direction. 
We can also view the noise floor in each chamber for the first time. The noise floor is the level at which the VNA, given its current settings, cannot detect below. Ideally the signal to noise ratio (SNR) will be very high. That being said, the measurements from both chambers for this antenna are both mostly above their respective noise floor. The noise floor in the Hangar Lab chamber is much lower, with less variance than that of the Microwave Lab chamber, averaging $-85.6 \mathrm{~dB}$ with a maximum of $-80 \mathrm{~dB}$, while that of the latter averaged $-75.5 \mathrm{~dB}$ with a maximum of $-64.4 \mathrm{~dB}$. What this means is that any recorded signal below the maximum noise level has to be thrown out. The data from the Microwave lab dropped below its noise floor maximum in a few places in the side lobes, so those data points are not valid. It is good that the Microwave Lab chamber has a shorter antenna separation distance because the increased signal level compensates for the relatively higher noise floor there. 


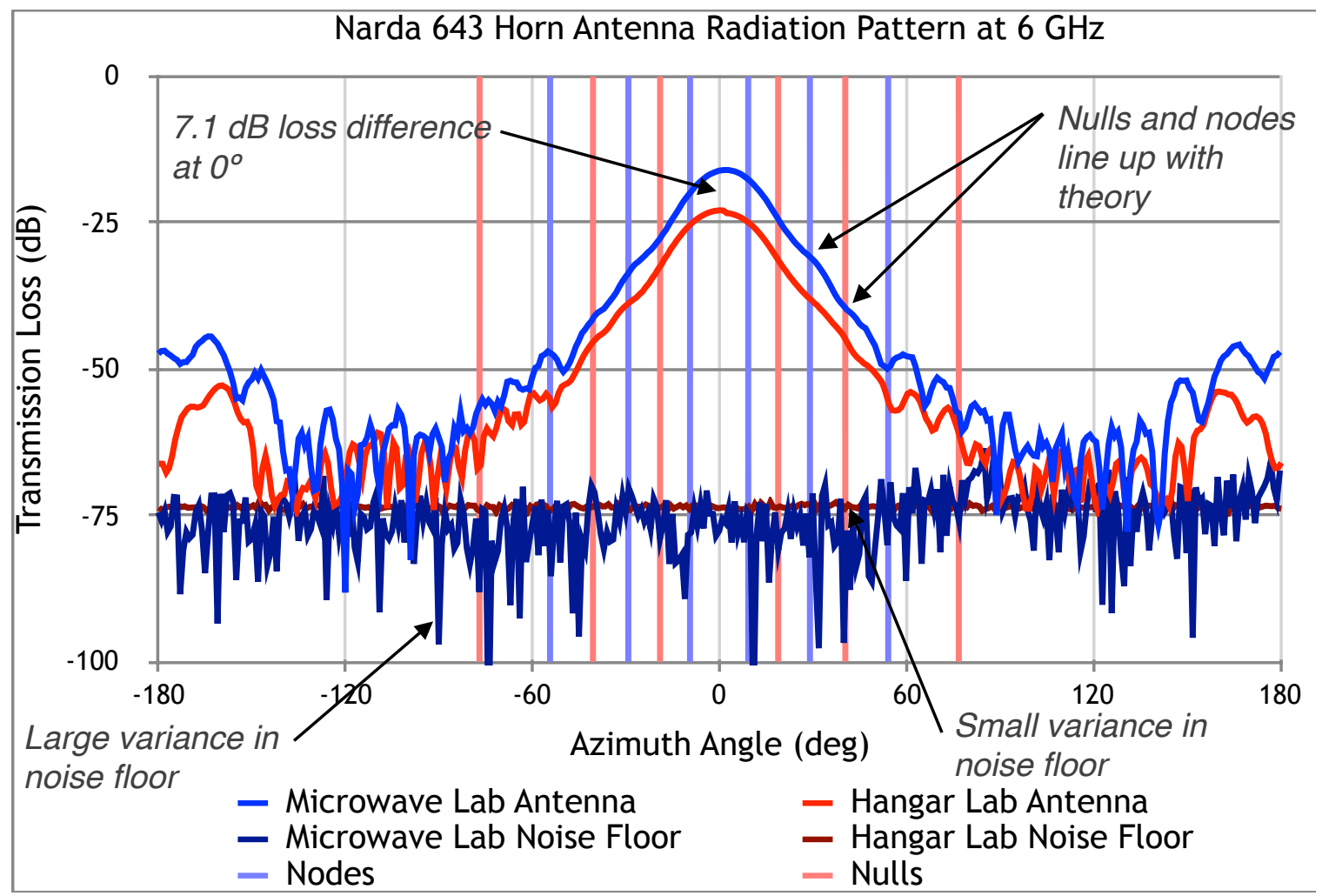

Figure 26 XY Scatter Plot of Narda 643 Horn Antenna Pattern Measured in Both

Chambers with Noise Floor, Nodes and Nulls

Looking now at the pattern of the Narda 643 horn, we can see the comparison is similar to that of the 642 horn. The Microwave Lab system recorded a $7.1 \mathrm{~dB}$ loss difference at $0^{\circ}$ from the Hangar Lab system, once again in line with our estimation of 8 $\mathrm{dB}$. The nulls and nodes for both chambers line up well with the estimated pattern nulls and nodes. Note that these nulls are newly calculated for this antenna at $6 \mathrm{GHz}$, as the antenna was previously measured at $5 \mathrm{GHz}$. Here we can also see the noise floor for both chambers creeping up to around $-75 \mathrm{~dB}$, but still well below most of the recorded antenna patterns. We also see good symmetry in both chambers, however the patterns begin to differ more around the rear facing side-lobes. Some of these differences could be due to differences in the mounting hardware used in each antenna, as these begin to have effect at lower power levels. 


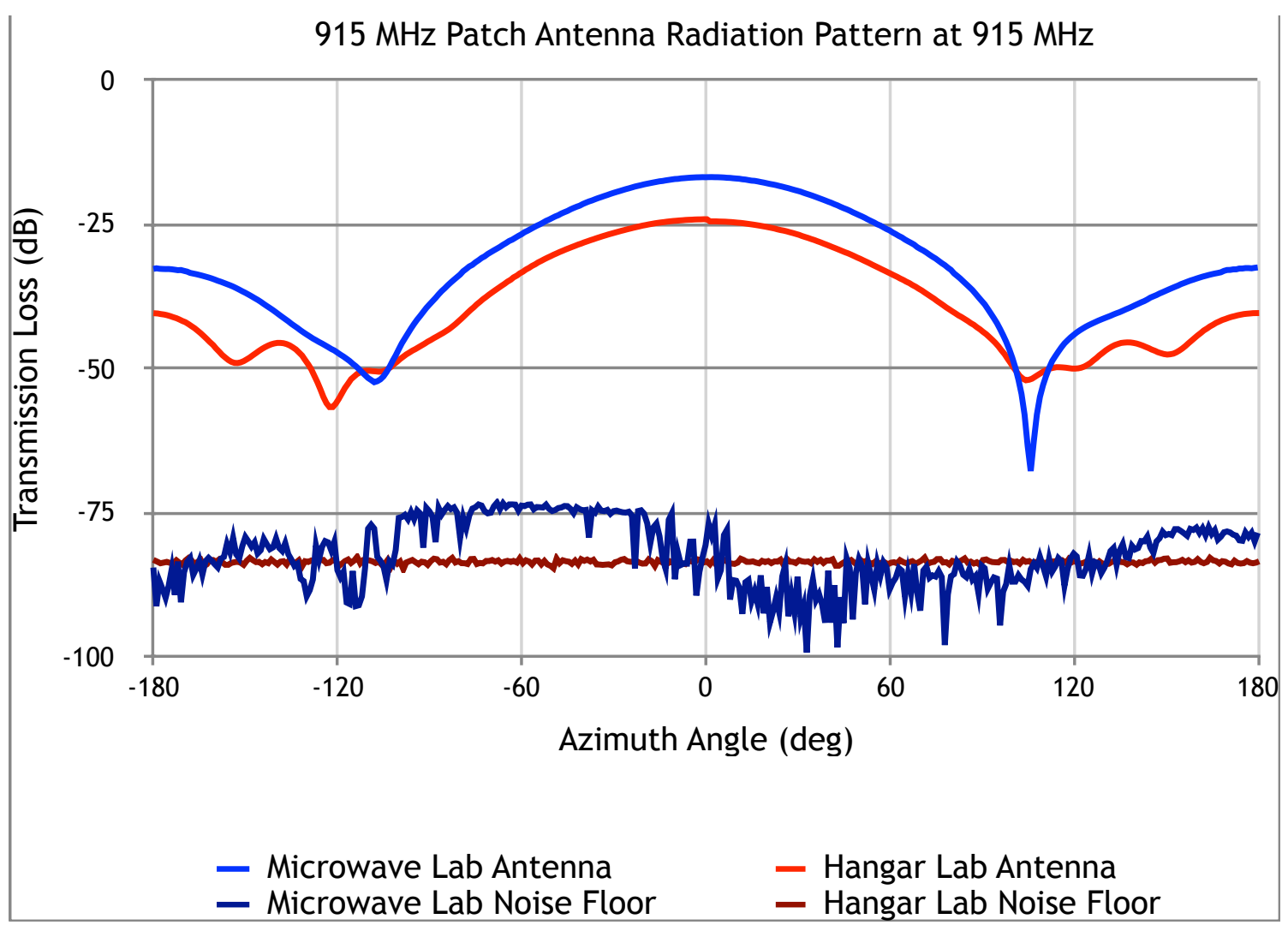

Figure 27 XY Scatter Plot of 915 MHz Patch Antenna Pattern Measured in Both

Chambers with Noise Floor

Finally, the $915 \mathrm{MHz}$ patch is the last antenna remeasured, this is because the broadband horn was no longer available. We expect to see more of the effects of using a shorter antenna separation distance with this antenna because of the relatively low frequency and longer wavelength compared with that of the Narda horns. As noted earlier in section 4.1, a smaller chamber has a shorter antenna separation distance and this means the antenna doing the detection may not be sufficiently in the far field zone of the AUT. The effects of this are that the signals from the AUT are not approximately planar, resulting in a more smoothed out antenna pattern. Less detail, for example in the side lobes, of the antenna pattern will be visible. Looking at the results for the $915 \mathrm{MHz}$ patch antenna, this is shown to be true. The Microwave Lab data, although $7.5 \mathrm{~dB}$ 
stronger at $0^{\circ}$, shows only two distinct lobes: a primary beam and a reverse lobe. Whereas the Hangar Lab, with its greater separation distance, records six: the primary lobe, two new side-lobes, and a reverse lobe.

This is an interesting observation and gives additional insight and proof into the benefits of a larger chamber. Although the Hangar Lab chamber is slightly less sensitive at detecting a signal $(7.5 \mathrm{~dB})$, what it lacks in overall sensitivity, it makes up for in the detail of side the lobes that the smaller chamber could not detect. The trade off in this particular case paid off. The downside of less power reaching the RF probe due to a larger antenna separation distance is that the recorded signal is then less powerful relative to the noise floor, reducing SNR. However, since in this case the noise floor in the Hangar Lab was well below $(26.9 \mathrm{~dB})$ the lowest recorded point of the antenna pattern there, there was plenty of overhead to increase the antenna separation distance even more. 


\section{CHAPTER 5: Conclusion}

Overall, the new Microwave Lab Antenna Measurement System measures antenna patterns with good accuracy at close range all while being easy to operate. Relative to its predecessor, the Hangar Lab AMS, it does some things better and some things worse. While both chambers accurately record pattern nulls and nodes within $10^{\circ}$ of their estimated positions, the Microwave Lab system still struggled at the low frequency with the $915 \mathrm{MHz}$ patch, due to its shorter antenna separation distance. At higher frequencies, above $5.5 \mathrm{GHz}$, the detail in the side lobes recorded by the Microwave Lab system were on par with the larger Hangar Lab system. That is not to say the Microwave Lab system is not going to be accurate at frequencies below 5.5

$\mathrm{GHz}$, but rather the user should always estimate the far field distance of their AUT beforehand to decide if the larger chamber is necessary.

The Hangar Lab also consistently showed a lower noise floor than the Microwave Lab, meaning the $20 \mathrm{GHz}$ HP VNA there has much greater sensitivity than the Anritsu VNAs available in the Microwave Lab. This plays to the advantage of the Hangar Lab chamber, being that it is of a bigger size it needs the extra sensitivity to detect lower level signals that have decayed much more on their trip from the AUT to the receive antenna. It should be noted that the $6 \mathrm{GHz} \mathrm{HP}$ VNA available in the Microwave Lab had a much higher noise floor than the Anritsu models, and is thus not recommended for use nor was it used in this comparison. Although the Microwave Lab exhibited a higher noise floor in this comparison, the SNR was always greater than one over most of the swept angles for each antenna.

In addition to measurement accuracy, the new system also includes more extensive data post processing, and a more accurate antenna positioner. The compiled 
LabVIEW executable makes it easy for students to access and use, and the proximity of the chamber to the rest of campus means it will get used more frequently.

Table 8 Summary of Expected vs Observed Differences and Similarities Between Hangar Lab Chamber and Microwave Lab Chamber

\begin{tabular}{|l|l|}
\hline Expectation & Observation \\
\hline $\begin{array}{l}\text { The Hangar Lab would have better } \\
\text { side lobe separation than the } \\
\text { Microwave Lab for lower frequency } \\
\text { antennas. }\end{array}$ & $\begin{array}{l}\text { The Hangar lab did indeed have better } \\
\text { side lobe separation for the } 915 \mathrm{MHz} \\
\text { patch, while side-lobe separation was } \\
\text { similar between the two chambers for } \\
\text { the higher frequency horn antennas. }\end{array}$ \\
\hline $\begin{array}{l}\text { The Microwave Lab would have a } \\
\text { similar noise floor level to the Hangar } \\
\text { Lab. }\end{array}$ & $\begin{array}{l}\text { The Microwave Lab, with its } 6 \mathrm{GHz} \\
\text { Anritsu VNA, had a higher noise floor } \\
\text { with a greater variance than the } \\
\text { Hangar Lab with its } 20 \text { GHz HP VNA. }\end{array}$ \\
\hline $\begin{array}{l}\text { The Microwave Lab would have } 8 \mathrm{~dB} \\
\text { less transmission loss than the Hangar } \\
\text { Lab due to its shorter antenna } \\
\text { separation distance. }\end{array}$ & $\begin{array}{l}\text { The Microwave Lab actually measured } \\
\text { on average 7.37 dB greater signal } \\
\text { strength at boresight than the Hangar } \\
\text { Lab across the antennas measured. } \\
\text { This a reasonable margin of error } \\
\text { given the different antenna mounting } \\
\text { methods. }\end{array}$ \\
\hline The Microwave Lab would run \\
radiation pattern scans faster. & $\begin{array}{l}\text { The Anritsu VNA and Sunol Sciences } \\
\text { FS-121 in the Microwave Lab take } \\
\text { about 10 minutes to complete a } \\
\text { radiation pattern sweep, while the HP } \\
\text { VNA and Sunol Sciences ELAZ75 in } \\
\text { the Hangar Lab take about 30 } \\
\text { minutes. }\end{array}$ \\
\hline
\end{tabular}

To sum up, the Microwave Lab AMS is a good substitute for the Hangar Lab chamber when measuring smaller antennas with short far field distances. For larger antennas, and antennas with longer wavelengths, the Hangar Lab remains the best. The new AMS is an accurate and easy to use resource for science and engineering students to learn more about antenna theory and operation in a way that contributes to the Cal Poly "learn by doing" ethos. Even before the project's completion, students were already 
asking about and using the automated measurement system and expressing positive feedback. The ease of use and greater proximity to classrooms increases the likelihood of students and teachers using an anechoic chamber, and an antenna design experiment such as the one in the next chapter is a good way to get students started. Additionally, the new LabVIEW interface is packed with features and displays information in a way that helps students learn antenna theory.

\subsection{Future Improvements}

\subsubsection{Antenna Separation Distance Tests}

A good future experiment would be to measure an antenna in the larger Hangar Lab chamber, but with the separation distance equal to that used in the Microwave Lab Chamber. This would get rid of one more variable in the chamber-to-chamber comparison. It would show whether the side lobe separation and noise artifacts are dependent on the distance or the anechoic chamber environment. One step further in this experiment would be to compare the pattern of an antenna at various separation distances within the larger chamber, and plot them on one plot to show how the pattern changes with distance.

\subsubsection{Free Space Material Measurements}

Anechoic chambers may be used for purposes other than antenna measurement. It is possible to measure electric permittivity, magnetic permeability, and sheet impedance of sheets of dielectric material by placing them in an anechoic chamber between two antennas and measuring the S-parameters of this network. This method is outlined in Focused Beam Methods: Measuring Microwave Materials in Free Space by John W. Schultz, and requires dielectric lenses to focus antenna beams onto the 
dielectric material to minimize fringing affects at the edges of the material. Unfortunately, due to time and financial constraints, implementation of this system was not possible for this project and is therefore suggested for a future project.

\subsubsection{Swept Frequency Radiation Patterns}

It is possible, given a minor tweak in the LabVIEW code, for the AMS to measure radiation patterns for a list or range of frequencies. This would work by programming the VNA to measure a list of frequencies for each angle of rotation of the AUT, as opposed to a constant wavelength, and then plot multiple traces or a 3D mesh for a single $360^{\circ}$ sweep. This idea was not implemented because it would increase complexity in a system designed to be simple to use. The capability still exists for the user to run multiple $360^{\circ}$ sweeps for various frequencies of interest, however each frequency would require a new AUT sweep. The user can decrease sweep time by increasing the azimuthal step size, or decreasing the azimuthal span.

\subsubsection{Time Domain Gating}

Even though the anechoic chamber is designed to attenuate most microwave reflections before they reach the receive antenna, low power reflections still occur and can alter measurements. The vector network analyzer can filter out reflections by only sensing a specific time domain window, or gate, of the incoming signal and ignoring any parts of the signal that follow. This is known as time domain gating. Because reflections travel a longer path than direct signals, they take longer to arrive at the receiving end. For the new anechoic chamber, this amounts to a few nanoseconds of extra travel time.

The Anritsu network analyzers are, at best, capable of a 10-ns gate. Given that

the antennas in the chamber are no more than $1.5 \mathrm{~m}$ away from each other, the 
maximum time for direct transmission is approximately $5 \mathrm{~ns}$. While some noise reduction was apparent with a $10 \mathrm{~ns}$ gate, possibly due to the elimination of secondary and tertiary reflections, more research and testing are required to determine what time domain gate would best eliminate primary reflections while preserving the complete direct transmission signal. One possible method for identifying reflections from direct path transmissions would be to configure the network analyzer to display signals in the time domain, and noting what time range the main burst of energy arrives. Furthermore, the AMS program could automatically adjust the time gate for different sized antennas, different antenna positioners, and different antenna angles. However, to keep the system easy to use, and to focus on other aspects of the AMS, this feature was left out. The user can still set up time domain gating manually before taking measurements if desired.

\subsubsection{Chamber Calibration for Active Measurements}

While the focus of this project was to establish a basic system capable of passive antenna measurements, a highly useful addition would be to calibrate the anechoic chamber for active wireless measurements. Active measurements involve testing an antenna as part of a system that includes a power source, amplifiers, and other wireless radio circuitry. Calibrating the chamber means quantifying the air loss between the DUT and the receive antenna as well as the loss in the coaxial cables running to and from any measurement equipment. With these values known, the system can calculate total radiated power (TRP) or total isotropic sensitivity (TIS) while considering desensitization of the antenna due to noisy components of the total system. These are industry standard measurements for wireless communication devices and students would benefit from learning more about them. 


\section{CHAPTER 6: Creating the Antenna Design Experiment}

Appendix $\mathrm{H}$ contains a laboratory experiment designed to introduce students to using the antenna measurement system. The intention is for the lab to be part EE533 Antennas course, and it references most of its material from the course textbook Antenna Theory and Design. The lab centers around designing, modeling, prototyping, and testing a pyramidal horn antenna, but students can use the lab as a guide to build any antenna they want. The goal of the lab is to get students comfortable with the anechoic chamber and give them hands on experience with antennas.

\subsection{Designing the Experiment}

Antenna design and test laboratory experiments must specify the test equipment's frequency range, antenna physical size, and practical antenna test procedures. This tutorial describes a $2.4 \mathrm{GHz}$ pyramidal horn antenna design. The 2.4 $\mathrm{GHz}$ band covers Wi-Fi and Bluetooth, two standards of interest to students, and is within chamber and test equipment frequency limits.

Because horn antennas require more time to construct than a milled patch antenna, students will require more than one session for experiment completion. Requiring all students to build and test their own antennas requires substantial materials and more than one test session. To reduce time and resources, each student models an antenna, but then works in groups of three for antenna fabrication. The experiment takes three days: a day to model, a day to construct, and a day to test. 


\subsection{The Design}

Students will use design equations from section 9.4.3 The Pyramidal Horn Antenna in Stutzman to design an antenna that results in practical physical dimensions. Given the standard waveguide dimensions for that frequency range, students should choose a gain value that yields a physically realizable ' $A$ ' dimension.

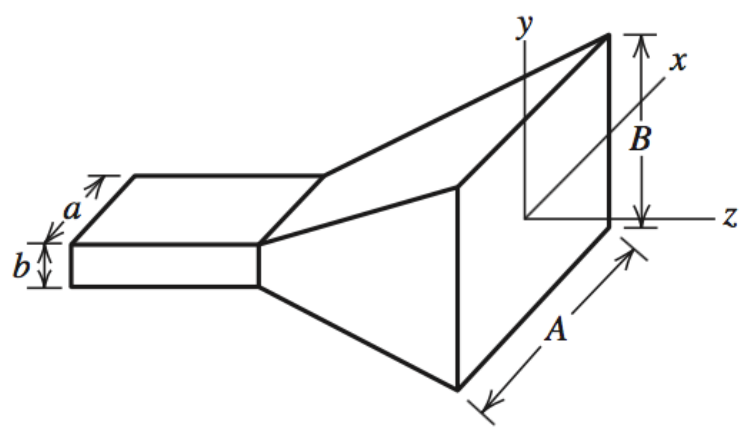

(a) Overall geometry.

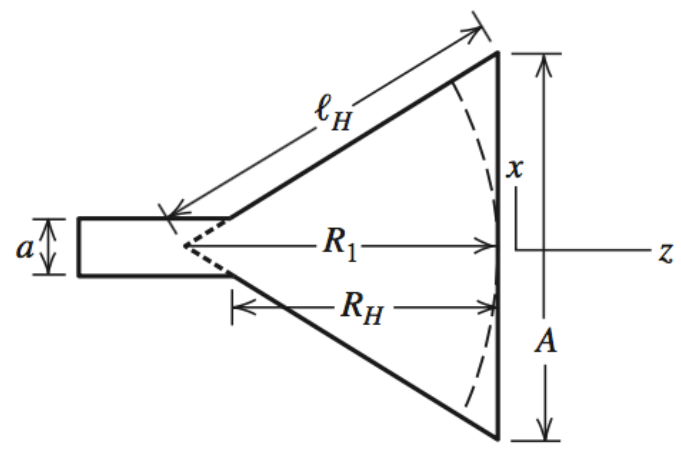

(b) Cross section through the $x z$-plane ( $H$-plane).

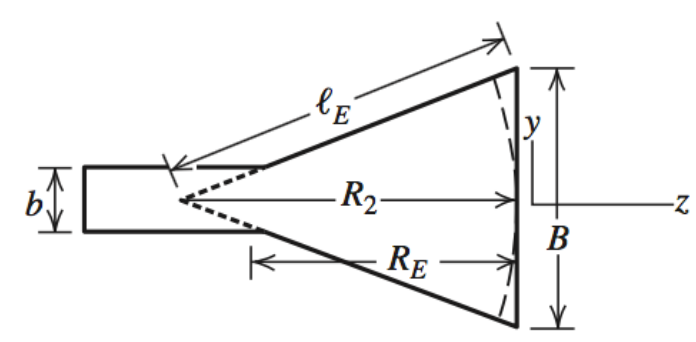

(c) Cross section through the $y z$-plane ( $E$-plane).

Figure 28 Pyramidal Horn Antenna Dimensions [1]

Section 9.4.3 in Stutzman contains all the necessary design equations and background for the design, as well as an example design and a method to verify the correct solution. The result should be a value for $R_{P}=R_{E}=R_{H}$ in cm. 
Below is a table of the standard waveguide sizes relevant to the $2.4 \mathrm{GHz}$ range. The S Band is on the list because although it does not specifically cover $2.4 \mathrm{GHz}$ it is the waveguide standard for the Narda 644 standard gain horn which will act as the source antenna when testing the students' antennas.

Table 9 Standard Rectangular Waveguide Dimensions [13]

\begin{tabular}{|l|l|l|l|}
\hline Frequency Band & $\begin{array}{l}\text { Waveguide } \\
\text { Standard }\end{array}$ & $\begin{array}{l}\text { Frequency Limits } \\
\text { (GHz) }\end{array}$ & $\begin{array}{l}\text { Inside Dimensions } \\
\text { (mm) }\end{array}$ \\
\hline R Band & WR-430 & 1.70 to 2.60 & $109.22 \times 54.61$ \\
\hline D Band & WR-340 & 2.20 to 3.30 & $86.36 \times 43.18$ \\
\hline S Band & WR-284 & 2.60 to 3.95 & $72.136 \times 34.036$ \\
\hline
\end{tabular}




\subsection{Modeling}

Once the students have their design written down, they can follow the tutorial in Appendix $\mathrm{H}$ to model their horn in HFSS. The tutorial is a bare bones guide to getting the horn design on the computer quickly. The guide allows students to input their own dimensions parametrically, making each design unique. After modeling, students will produce 3D polar radiation plots and rectangular phi cut radiation plots which they then export to a tab-delimited text file to compare to their physical design results. Figure 28 below shows an example of the final computer model. The antenna points along the $x$-axis to reflect how it will sit in the anechoic chamber.

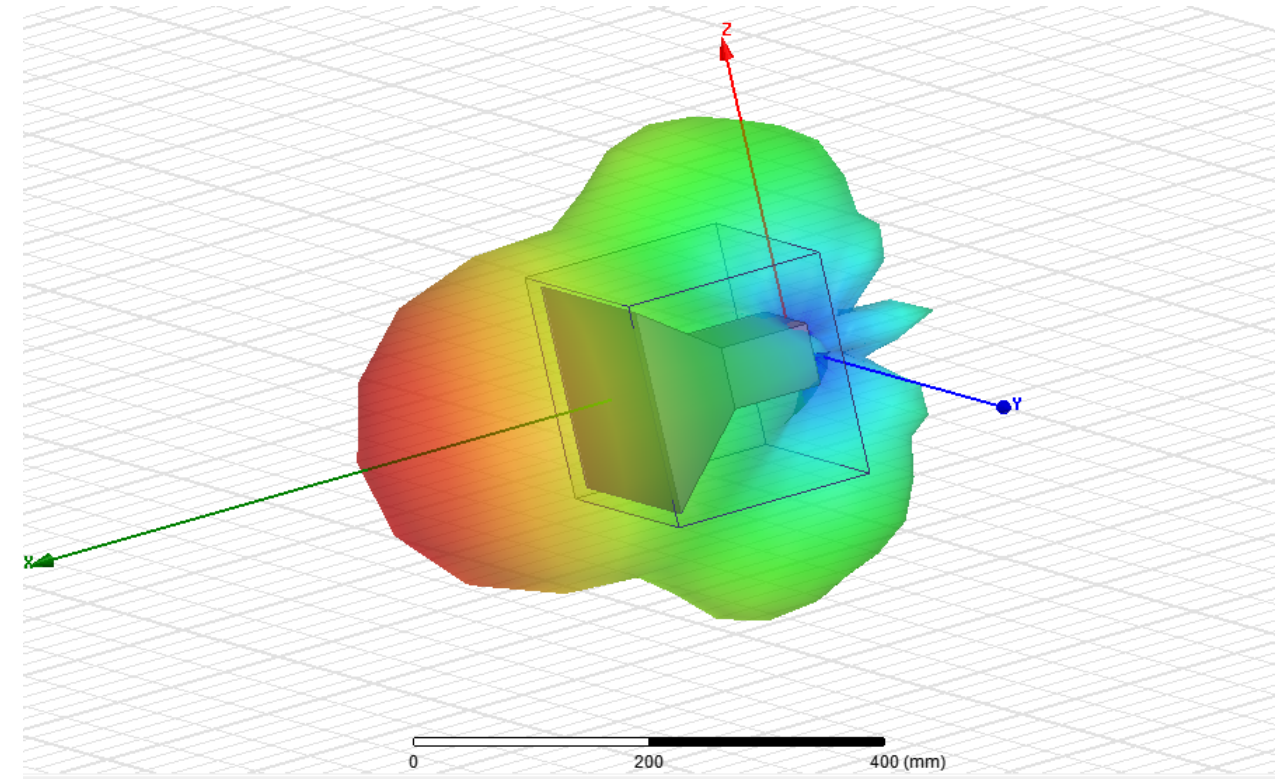

Figure $292.4 \mathrm{GHz}$ Pyramidal Horn Model in HFSS

\subsection{Prototyping}

After modeling, students in groups of three will use copper clad board to build their best design. Rogers Corporation provides free samples of their Duroid and FR4 for student projects through their university sample program, and can source the materials for the experiment. Since the design equations from Stutzman yield a value for $R_{P}$, the 
length of the horn in the $\mathrm{x}$-dimension, students need an equation to find the length of the face of each panel. The equation below uses the Pythagorean theorem to calculate the length of the $\mathrm{H}$-plane panels from $R_{P}, B$, and $b$, and will also calculate the length of the $\mathrm{E}$ plane panels by substituting in $A$ and $a$.

$$
\text { Length of Panel } A=\sqrt{R_{P}^{2}+\left(\frac{B-b}{2}\right)^{2}} \quad \text { Equation } 8
$$

Students will then cut the copper panels to size, and solder them together. The SMA connector should feed into a quarter wave monopole located in the center of the waveguide width and one quarter wavelength away from the back panel to produce in phase reflections. Figure 29 shows an example of what the final antenna might look like.
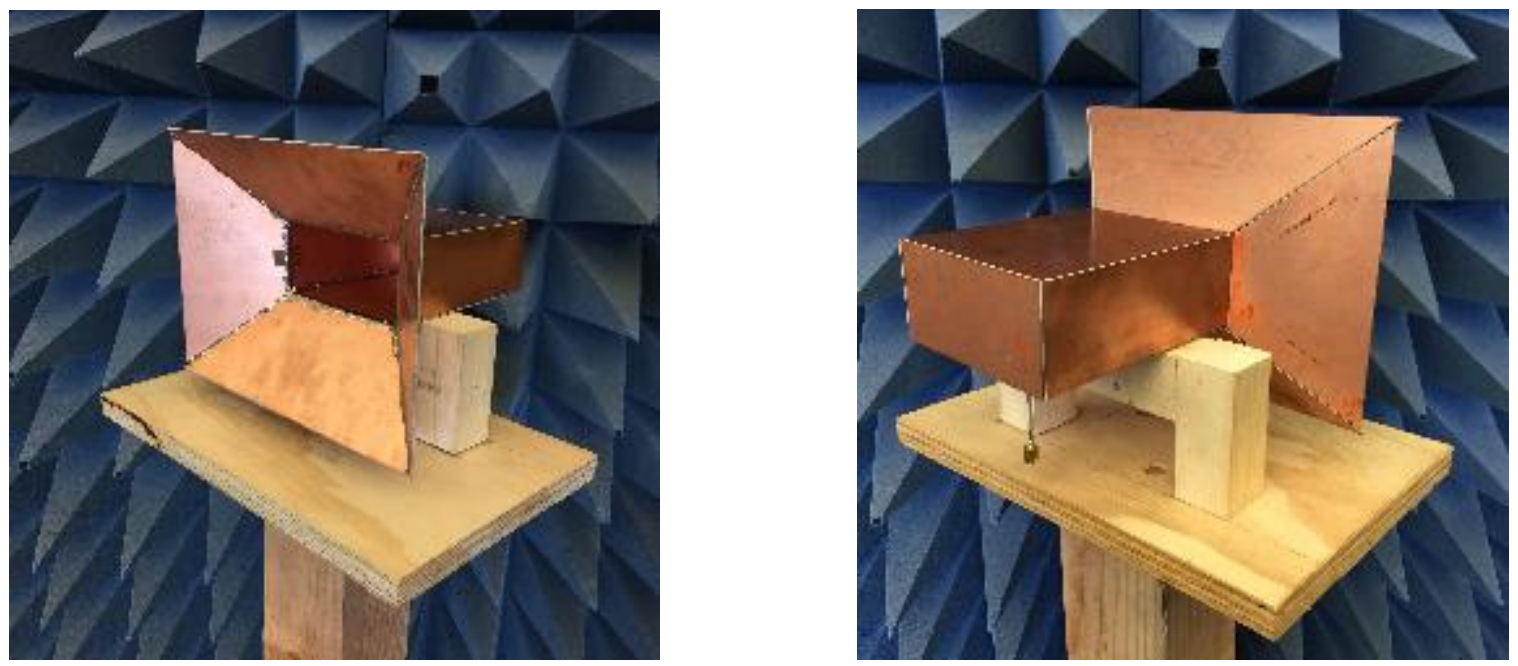

Figure 30 2.4 GHz Pyramidal Horn Antenna Prototype 


\subsection{Validating the Design Procedure}

Analysis of an antenna prototype designed for $12.6 \mathrm{~dB}$ gain at $2.4 \mathrm{GHz}$ confirms the design procedure produces a working antenna. Figure 29 shows this prototype. The HFSS model represents an ideal antenna. Its $\mathrm{S} 11$ and radiation pattern are meant to be design guides not actually achievable with a practical antenna. Figure 30 and 31 below compare the HFSS model with data from the prototype measured with the AMS.

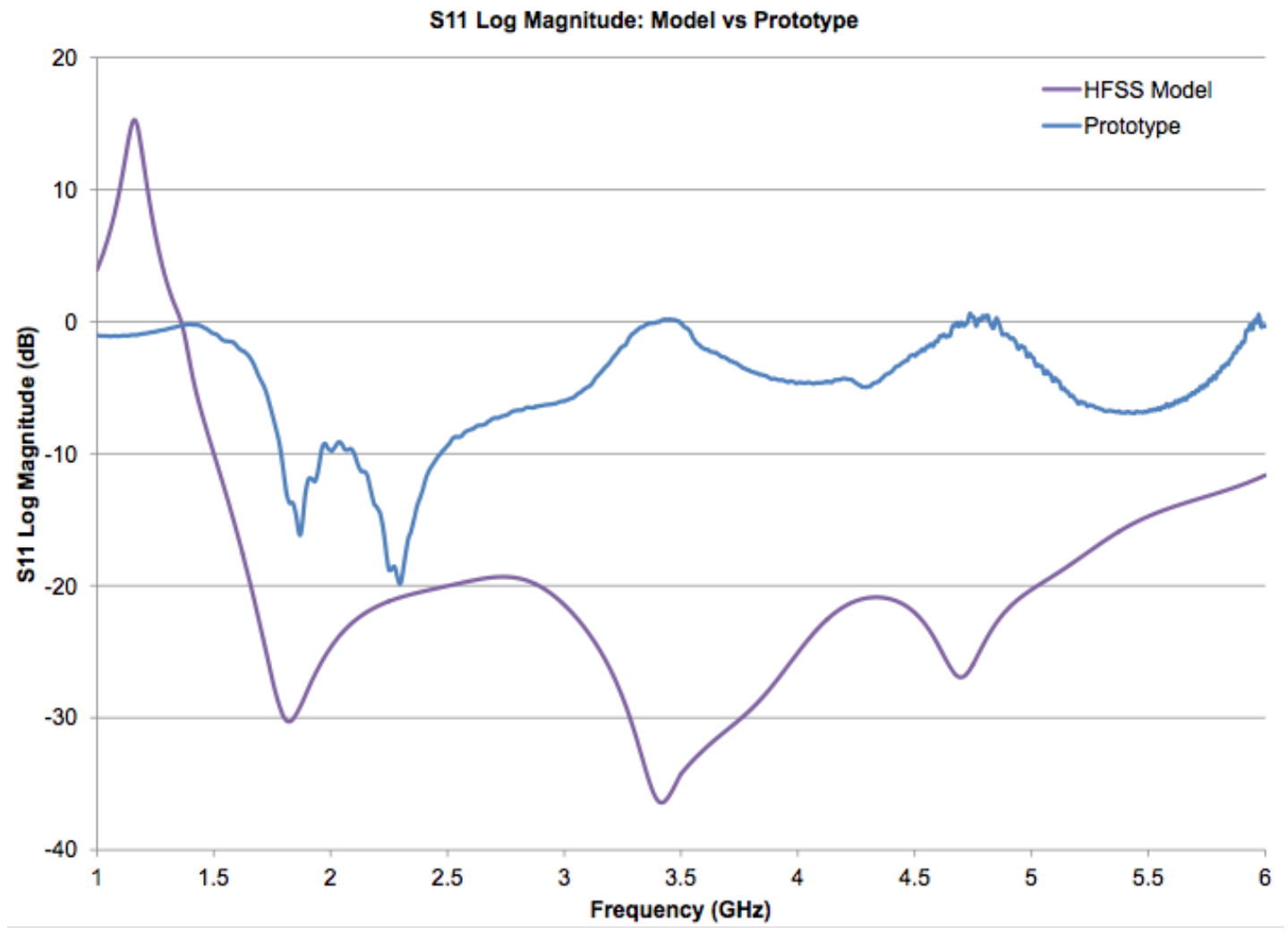

Figure 31 S11 Log Magnitude Plot Comparing HFSS Model to Prototype

S11 shows both the model and prototype below the VSWR threshold of 2, or $-9.54 \mathrm{~dB}$, at 2.4 GHz meaning the antenna will effectively radiate at this frequency. The model has a return loss of $20.42 \mathrm{~dB}$ and VSWR of 1.21 at $2.4 \mathrm{GHz}$ and the prototype has a worse return loss of $12.43 \mathrm{~dB}$ and VSWR of 1.63 at $2.4 \mathrm{GHz}$. The HFSS model shows an average rejection below $-20 \mathrm{~dB}$ throughout the 1.5 to $5 \mathrm{GHz}$ band, but the 
prototype does not match exactly, achieving a VSWR less than 2 only between 1.8 and 2.5 GHz. Nevertheless, the prototype still meets requirements for the $2.4 \mathrm{GHz}$ band.

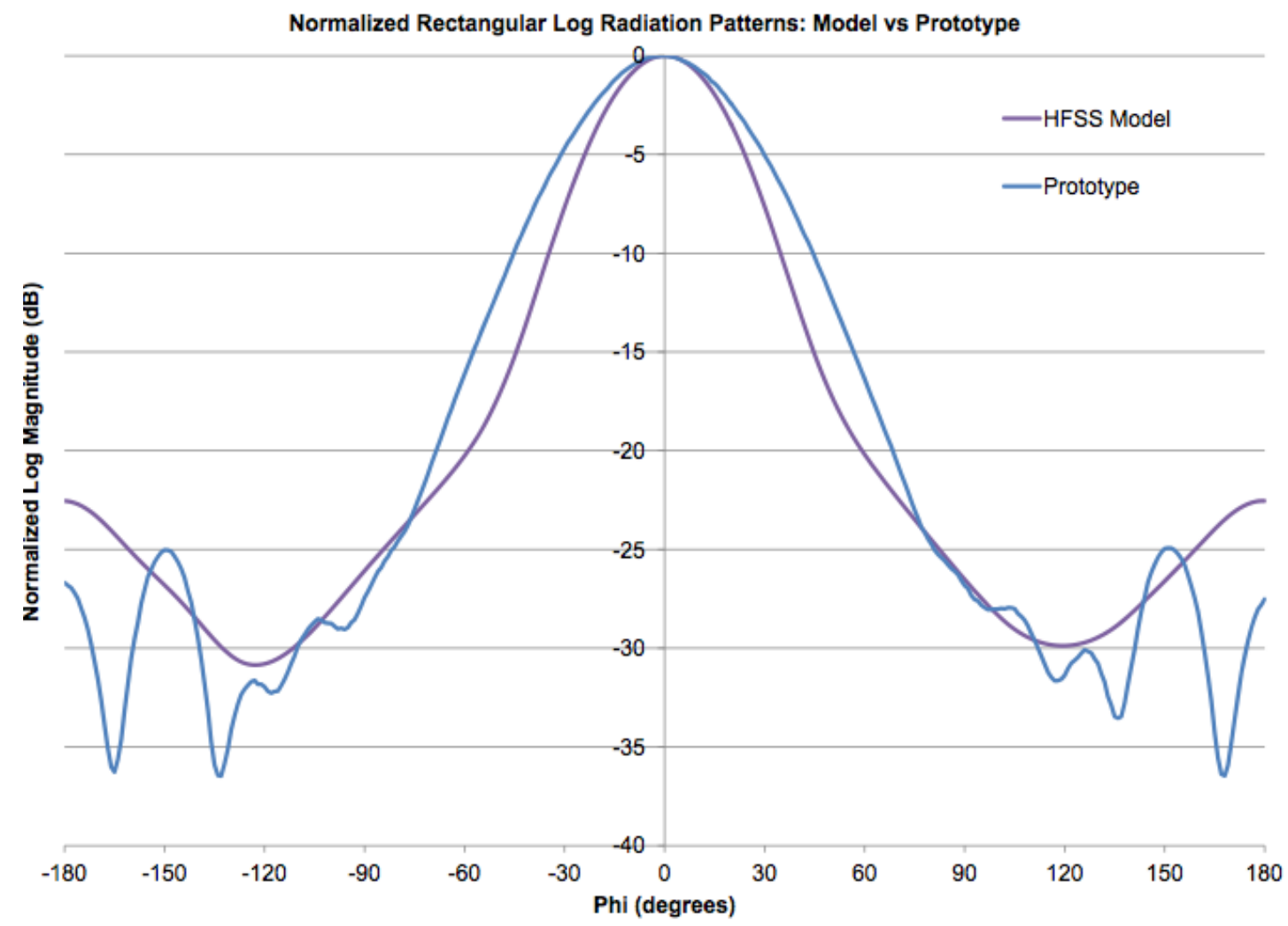

Figure 32 Normalized Rectangular Logarithmic Radiation Pattern Comparing HFSS Model to Prototype

The model's radiation pattern is smoother with a narrower main beam and a single back lobe, and the prototype has a slightly wider main beam with a pair of distinct side lobes at $\pm 150^{\circ}$ along and a smaller back lobe. Using the Directivity.m function discussed in section 3.6.2, the model horn has an estimated directivity of $18.35 \mathrm{~dB}$ and an HPBW of $38^{\circ}$, while the prototype has an estimated directivity of $12.06 \mathrm{~dB}$ and an HPBW of $47^{\circ}$. The prototype came considerably close to the original design goal of 12.6 $\mathrm{dB}$ gain, within less than $1 \mathrm{~dB}$, assuming 100 percent radiation efficiency. 
The lobes at $\pm 150^{\circ}$ in the prototype data are likely due to the sides of the horn flare transmitting unwanted radiation. These sides are facing normal to the receiver antenna at $\pm 150^{\circ}$. The model did not predict this because it assumes the materials are perfect electrical conductors, minimizing coupling. 


\section{BIBLIOGRAPHY}

(1) Stutzman, Antenna Theory and Design 3rd Edition

Section 9.4.3: The Pyramidal Horn Antenna describes the theory and design of pyramidal horn antennas.

Chapter 13 on antenna measurements has tons of information and images

(2) IEEE Standard Test Procedures for Antennas, ANSI/IEEE 149-1979

(3) Programming Manual for Agilent 37XXX Series VNA

http://downloadfile.anritsu.com/Files/en-US/Manuals/Programming-

Manual/10410-00301A.pdf?

f4739ea0f83b43ad1015d3937abcf8be6611e8da2557a55440e96cf4b1d03 c25e79240c4b49b81c163b3c09900fb6d85442efac31764eb813dce25e1cf 97a88aba004459db7be3b09e7c55dcfdc33d73e5153d3359e766ac7dba48 24022fd0f16d561e75ced49bc143cbb9cdd6c7\&10410-00301A.pdf

(4) Waveguide Flange Types and Materials, http://www.atmmicrowave.com/flange-size-type/

(5) Time Domain Analysis Using a Network Analyzer, http://cp.literature.agilent.com/litweb/pdf/5989-5723EN.pdf

(6) Analysis of gating errors in time domain antenna measurements, http://ieeexplore.ieee.org/xpl/freeabs_all.jsp?arnumber=35466

(7) Network Analyzer Basics, http://cp.literature.agilent.com/litweb/pdf/5965-7917E.pdf

(8) Radiation from Antennas http://elektroarsenal.net/radiation-fromantennas.html

Graphics including antenna coordinate system, and antenna power patterns on polar and rectangular plots

(9) Design and Implementation of a Pair of Horn Antenna for UIU Microwave Test Setup - Bangladesh

Contains a simple tutorial for modeling a $10 \mathrm{GHz}$ pyramidal horn antenna in an older version of HFSS.

Useful for the bulk of the modeling process, but does not cover Far-Field Radiation Setup.

(10) Getting Started with HFSS v9 for Antenna Design - Colorado Explains the meaning behind all the steps involved with modeling an antenna in HFSS.

Covers Far-Field Radiation Setup. 
(11) HFSS Online Help

Shows how to overlay a 3-D polar radiation plot on the original model in HFSS.

(12) High Performance Horn Antenna Design II

This presentation has lots of good pictures including: an excellent diagram of a pyramidal feed horn (p16), and images and graphics of conical and profile horns.

Also has some useful looking design equations for pyramidal horns.

(13) Rectangular Waveguide Dimensions http://www.microwaves101.com/encyclopedias/rectangular-waveguidedimensions

Table displaying all standard waveguide sizes (WR standard US)

(14) Rogers Corporation Website http://www.rogerscorp.com/index.aspx

(15) Waveguide Mathematics http://www.microwaves101.com/encyclopedias/waveguide-mathematics

(16) Radiation Pattern, Wikipedia http://en.wikipedia.org/wiki/Radiation pattern

(17) Anritsu, MS462XX Vector Network Systems Measurement Guide

(18) Sunol Sciences Corporation Website http://www.sunolsciences.com

(19) Sunol Sciences FS-121 Turntable Datasheet

(20) Toyon Corporation Website http://www.toyon.com

(21) VSWR Calculator, Microwaves101 http://www.microwaves101.com/encyclopedias/vswr-calculator

(22) National Instruments Website www.ni.com Information on LabVIEW. Source for LabVIEW instrument drivers.

(23) dirplot, Steve Rickman. Matlab Central, 20 Jan 2014. http://www.mathworks.com/matlabcentral/fileexchange/1251-dirplot

(24) DJM Electronics, EMI Filters and RF Shielding Products http:// www.djmelectronics.com/rf-absorber.html Information on RF Absorber material. Covers ferrite tile absorber and 
broadband foam absorber of various types. Absorber material available for purchase and quote. 


\section{APPENDIX A: Far Field Derivation}

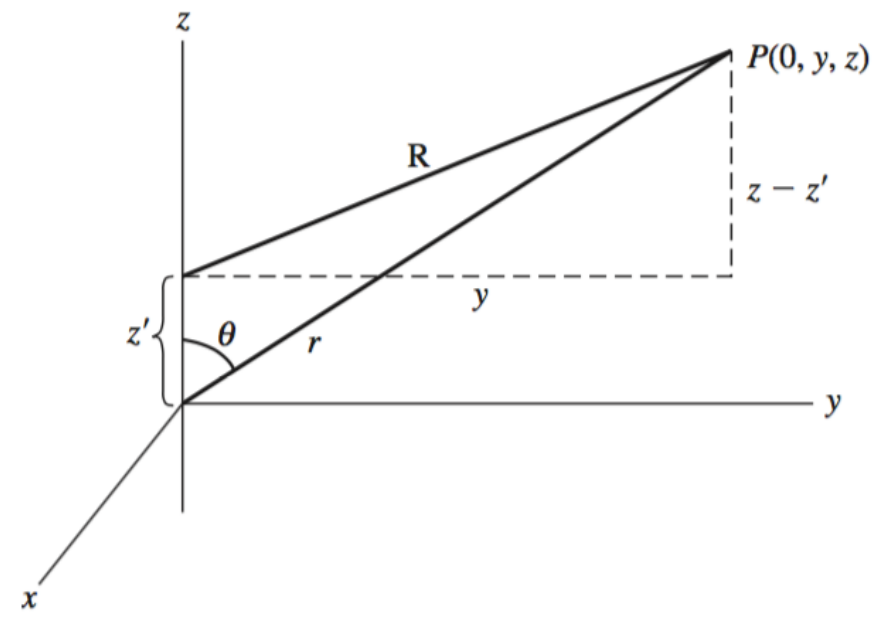

Figure 33 Geometry for Field Calculations of a Line Source

Given a line radiation source, at an observation point $P$ at a distance $r$ and angle $\theta$ from the center of the source, radiation along a ray $R$ drawn from the end of the source to the observation point will exhibit a phase shift compared to radiation along ray $r$. This phase shift will cause interference and one must consider this for observation points sufficiently close to the source. Length $R$ is calculated using the Pythagorean Theorem

$$
R=\sqrt{y^{2}+\left(z-z^{\prime}\right)^{2}}=\sqrt{y^{2}+z^{2}-2 z z^{\prime}+\left(z^{\prime}\right)^{2}}
$$

Putting all points into the spherical coordinate system, the equation translates to

$$
R=\left\{r^{2}+\left[-2 r \cos \theta z^{\prime}+\left(z^{\prime}\right)^{2}\right]\right\}^{1 / 2}
$$

To develop approximate expressions for $R$, expand using the binomial theorem

$$
\begin{aligned}
R= & \left(r^{2}\right)^{1 / 2}+\frac{1}{2}\left(r^{2}\right)^{-1 / 2}\left[-2 r \cos \theta z^{\prime}+\left(z^{\prime}\right)^{2}\right]+\frac{\frac{1}{2}\left(-\frac{1}{2}\right)}{2}\left(r^{2}\right)^{-3 / 2} \\
& \cdot\left[-2 r \cos \theta z^{\prime}+\left(z^{\prime}\right)^{2}\right]^{2}+\cdots \\
= & r-z^{\prime} \cos \theta+\frac{\left(z^{\prime}\right)^{2} \sin ^{2} \theta}{2 r}+\frac{\left(z^{\prime}\right)^{3} \sin ^{2} \theta \cos \theta}{2 r^{2}}+\cdots
\end{aligned}
$$




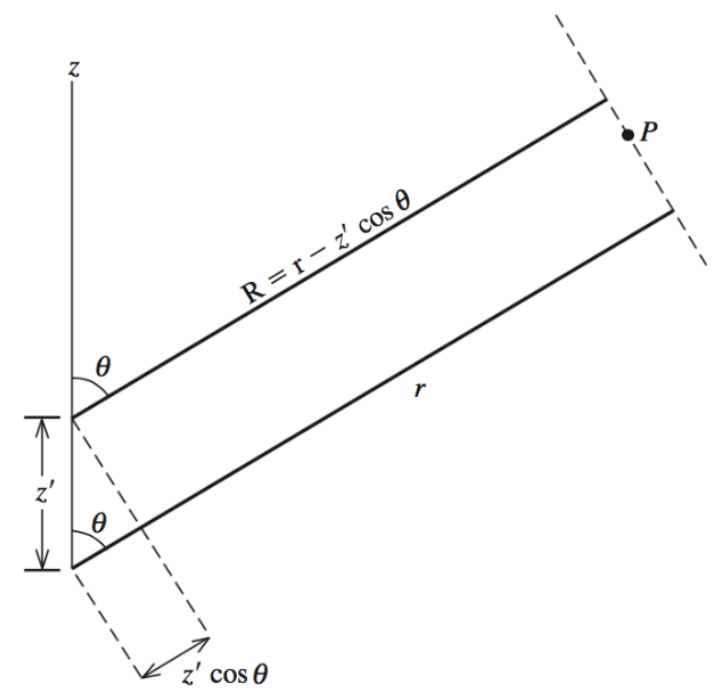

Figure 34 Parallel Ray Approximation for Far-Field Calculations of a Line Source

The Far Field is the range where the two rays are assumed parallel, or where the effects of the third term of the expanded equation for $R$ become negligible. This parallel ray approximation simplifies the calculation of the phase difference between the two rays. The third term of the expanded equation for $R$ is considered negligible when it less than or equal to a sixteenth of a wavelength. This corresponds to a phase error of

$$
\frac{2 \pi}{\lambda} * \frac{\lambda}{16}=\frac{\pi}{8} \mathrm{rad}=22.5^{\circ}
$$

Using $D$ as the length of the line source, the maximum value of the third term, or worst case scenario, occurs when $z^{\prime}=D / 2$ and $\theta=90^{\circ}$. Setting this maximum equal to a sixteenth of a wavelength gives an equation for the far field distance $r_{\mathrm{ft}}$.

$$
\frac{\left(\frac{D}{2}\right)^{2}}{2 r_{f f}}=\frac{\lambda}{16}
$$

Solving for $r_{f f}$ gives

$$
r_{\mathrm{ff}}=\frac{2 D^{2}}{\lambda}
$$


APPENDIX B: Custom Positioner Fixture Schematic

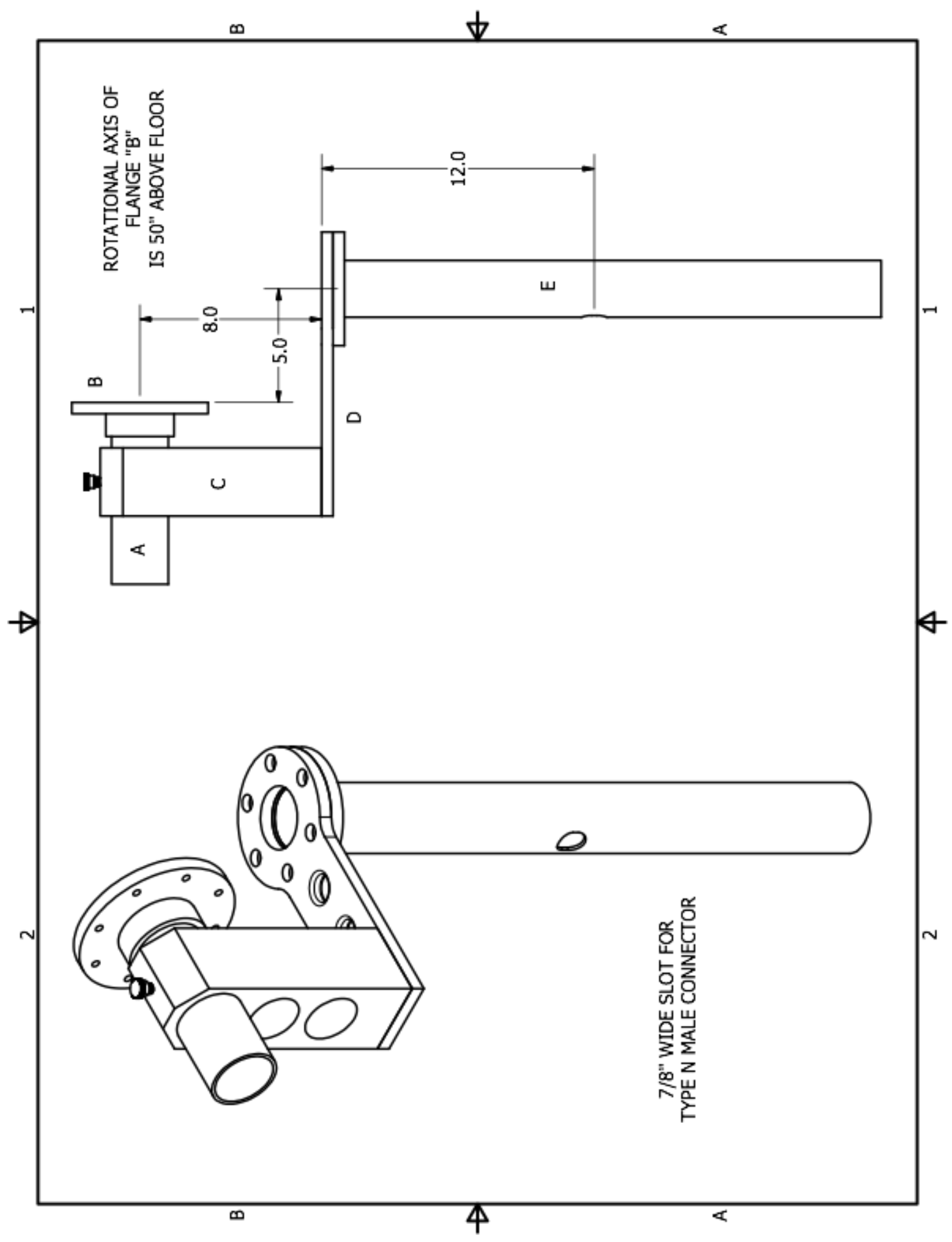


APPENDIX C: Receiver Antenna Fixture Schematic

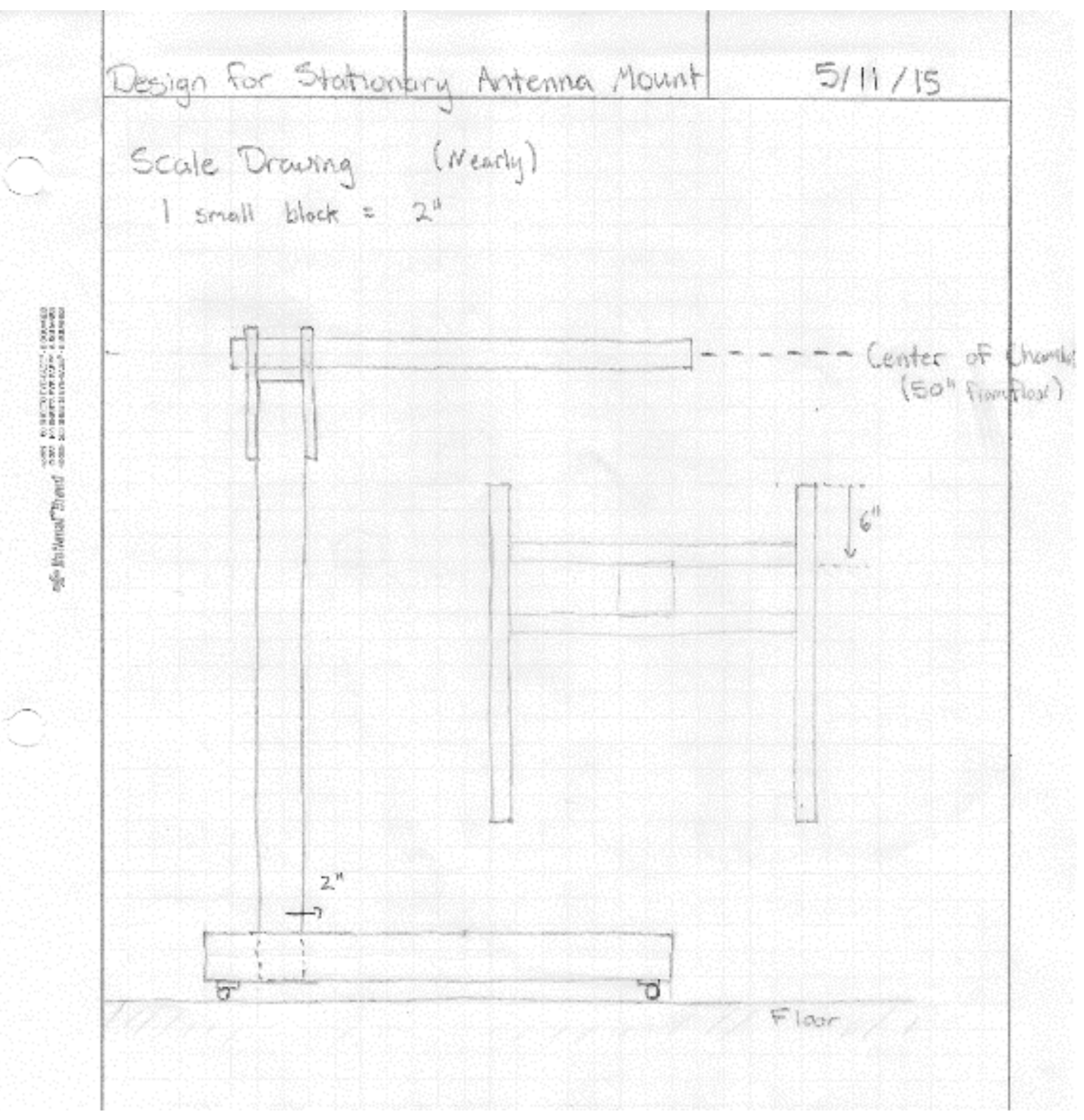




\section{APPENDIX D: LabVIEW AMS Flowchart}

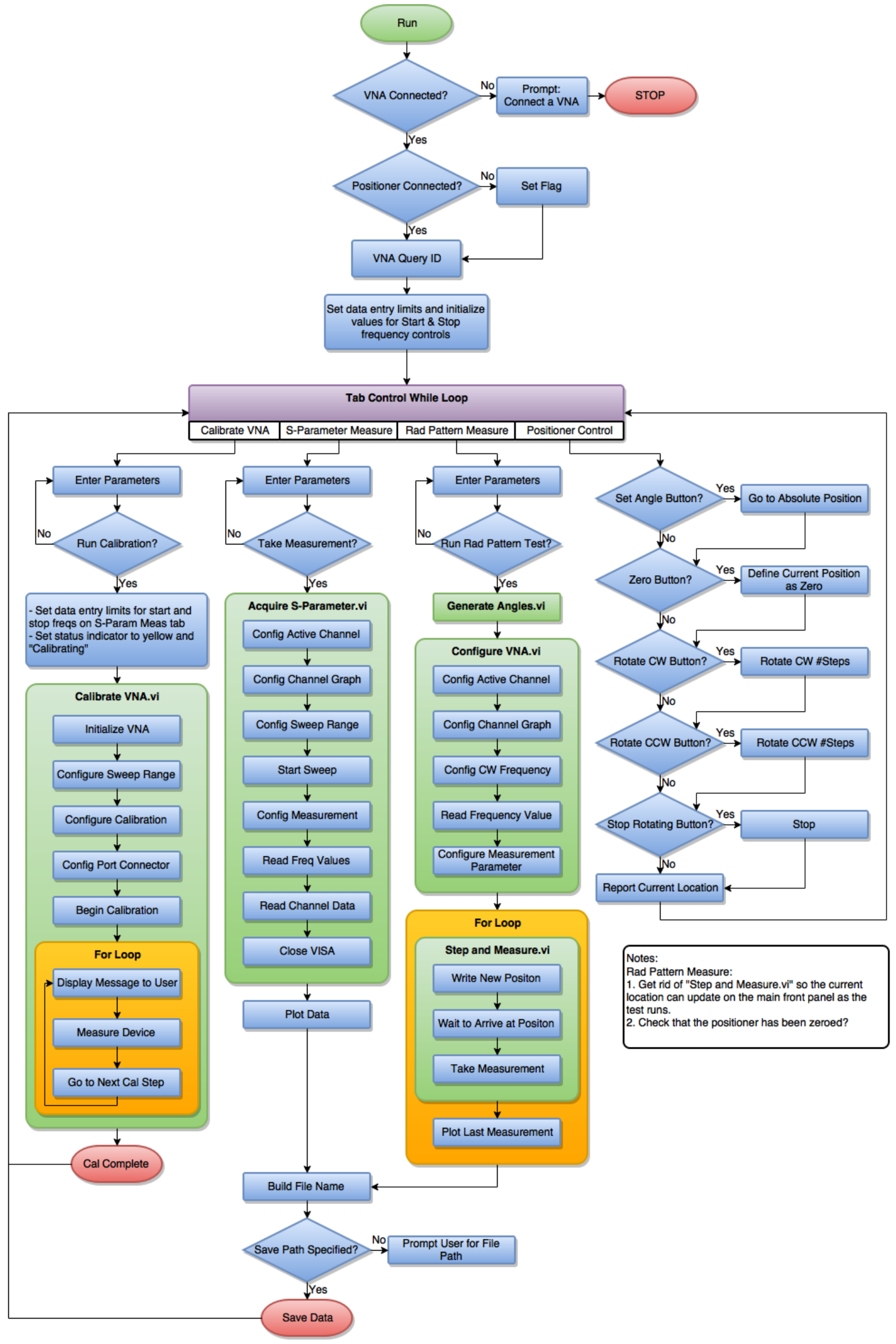




\section{LabVIEW Antenna Measurement System User Guide}

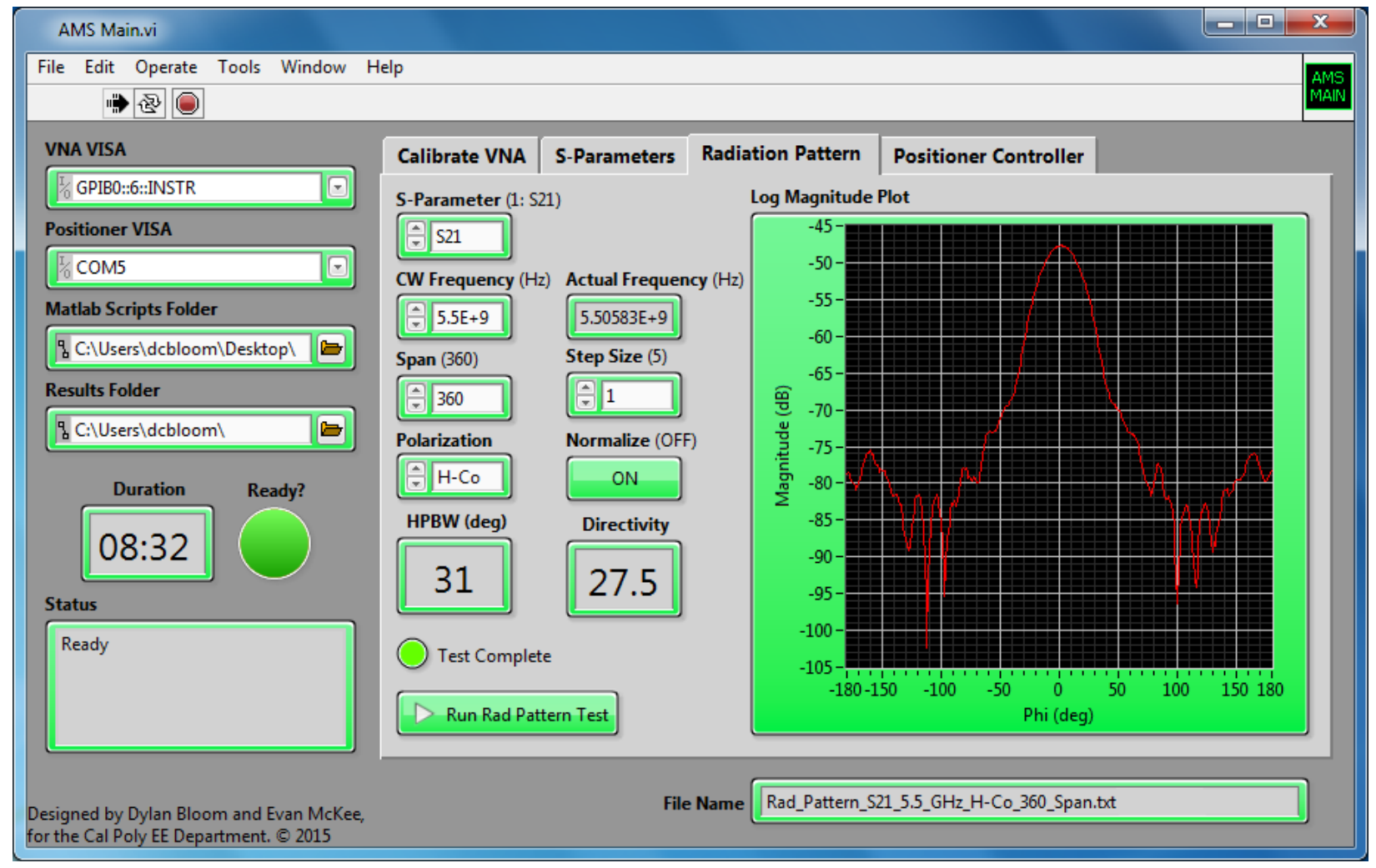

Cal Poly San Luis Obispo

Dylan Bloom Electrical Engineering Department June, 2015 


\section{Table of Contents}

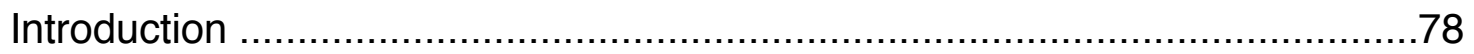

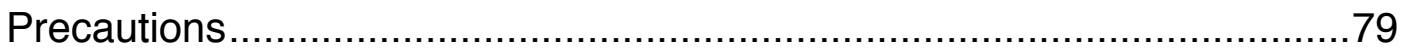

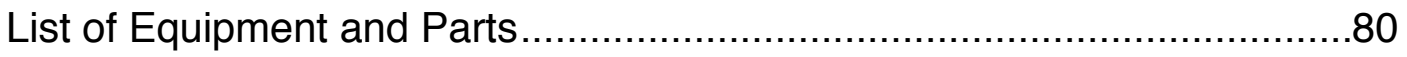

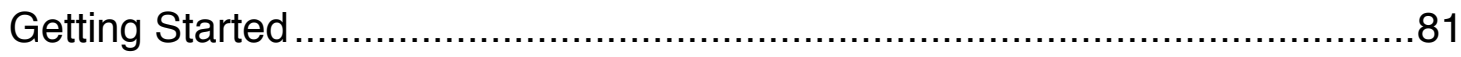

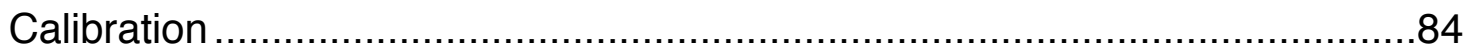

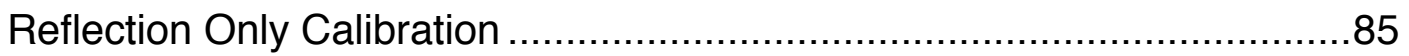

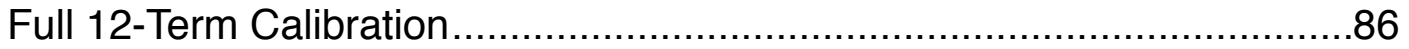

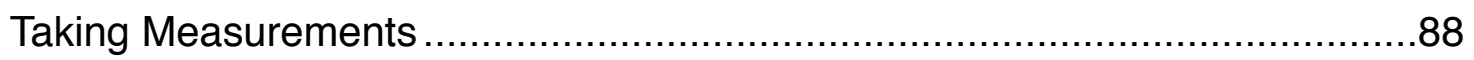

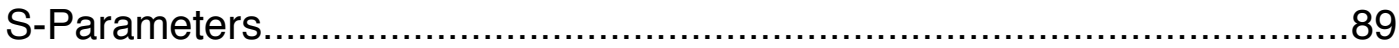

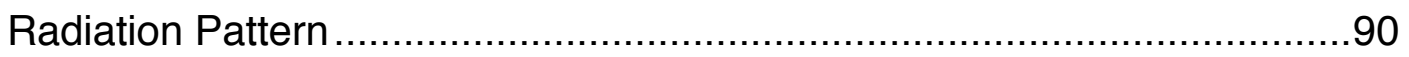




\section{Introduction}

The Microwave Lab Antenna Measurement System (AMS) allows Cal Poly students to measure antenna radiation patterns and s-parameters accurately and conveniently in a laboratory environment. This user guide is meant to be an introduction. It illustrates step-by-step how to make basic measurements. The methods in this guide can be extrapolated to more advanced methods such as the gain transfer method for measuring gain or the polarization pattern method for measuring polarization.

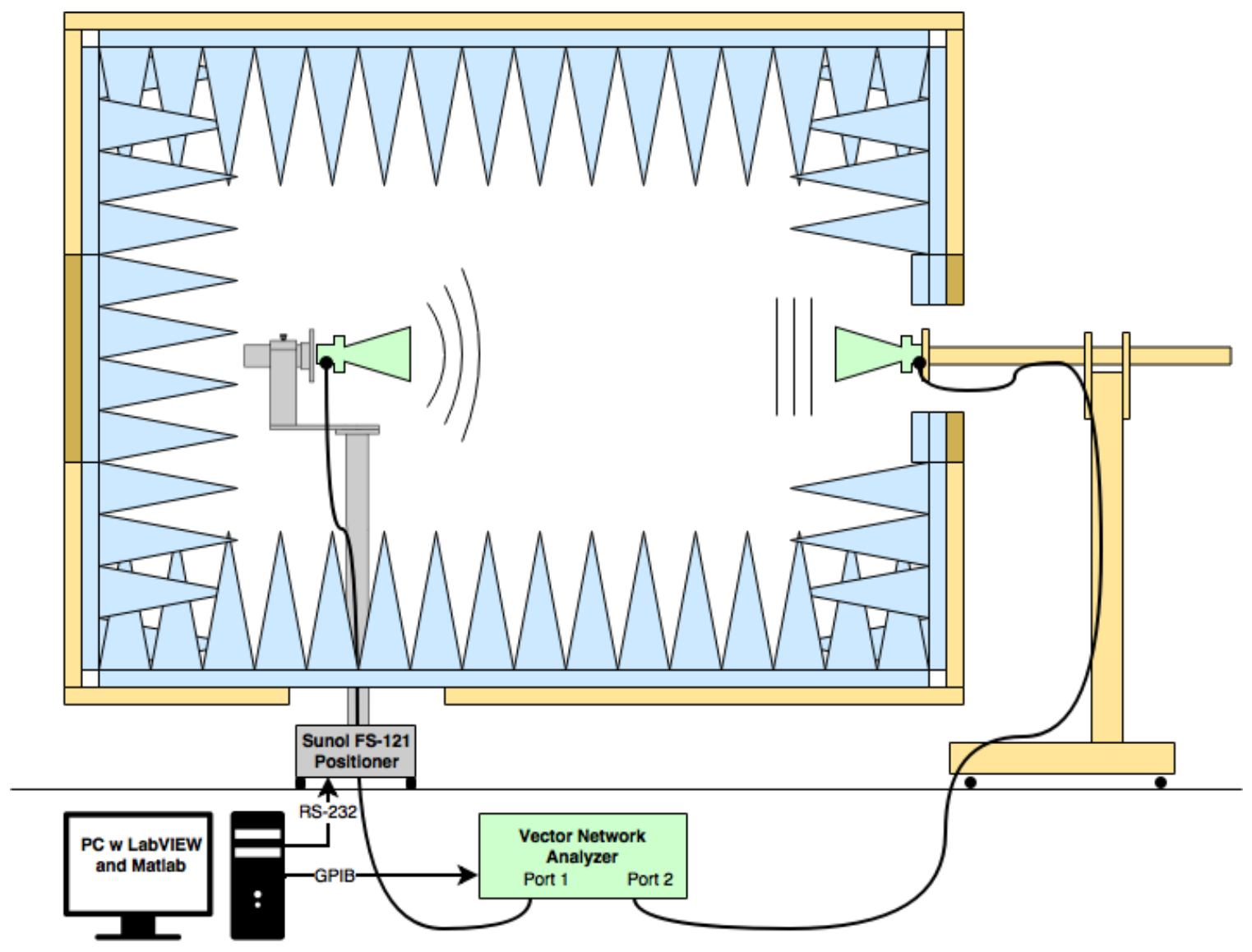

Figure 1 Drawing of the AMS 


\section{Precautions}

When working with the AMS, follow these precautions to avoid damaging any equipment.

1. Please avoid touching the foam. Deformations alter its absorptive properties and it can release particles into the air that are harmful if inhaled.

2. Use large radius bends when laying or coiling SMA cables. Sharp bends can damage them.

3. Use masking tape to hold cables in place, such as near where they connect to the VNA, or anywhere else they might move around or otherwise bend too sharply.

4. Get approval from Dr. Dean Arakaki before using the AMS for the first time. Dr. Arakaki oversees the chamber and can assist you with your design.

5. Schedule your time wisely so to not interfere with any lab sessions that may need the chamber.

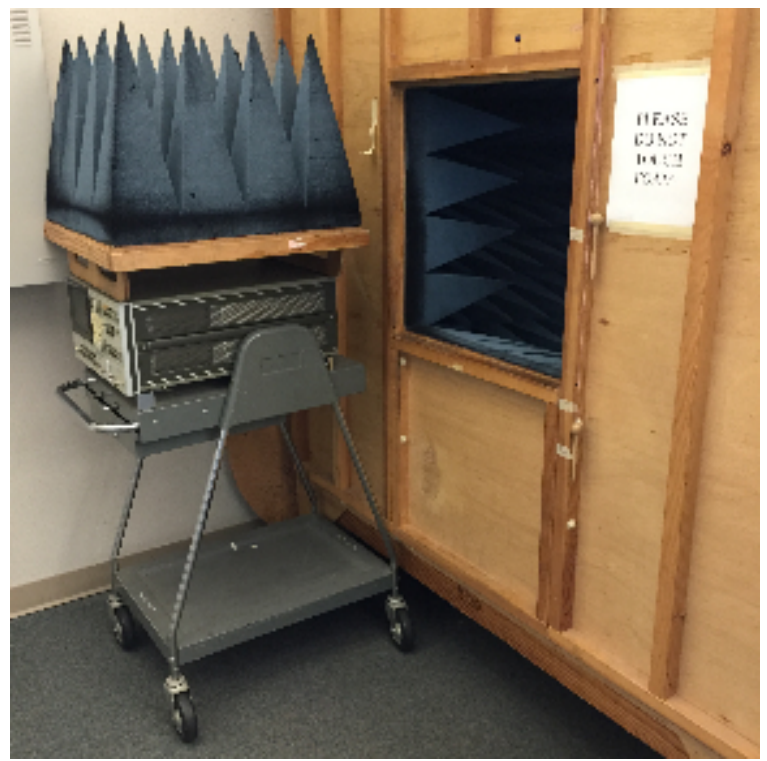

Figure 2 Chamber Window

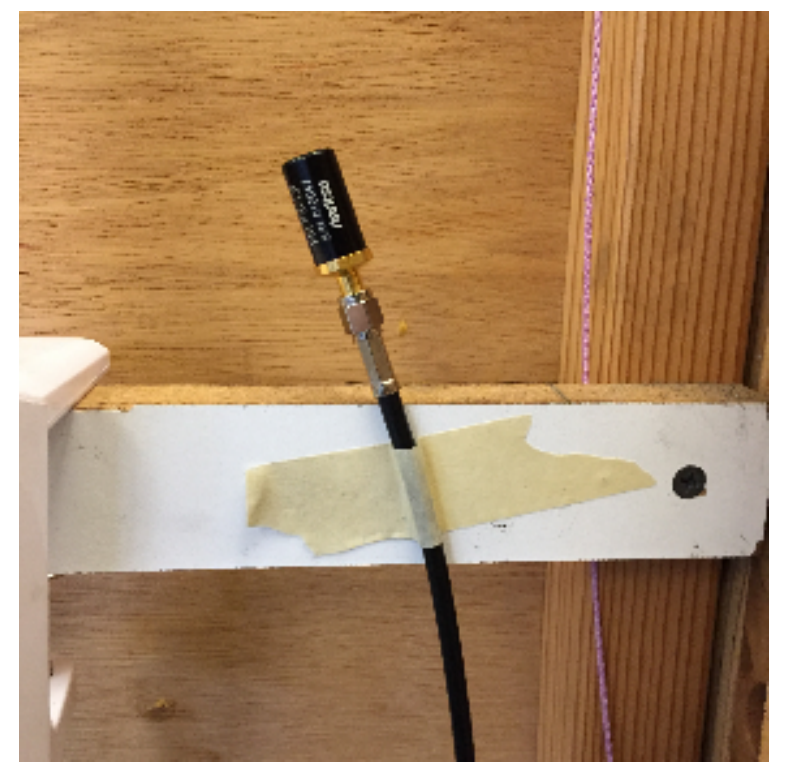

Figure 3 SMA Cable 


\section{List of Equipment and Parts}

The following instruments and parts are needed to run the tests in this guide.

- Vector Network Analyzer (VNA)

- Anritsu MS4623D Vector Network Analyzer (10 MHz - $6 \mathrm{GHz}$ )

- Anritsu MS4622B Vector Network Analyzer (10 MHz - $3 \mathrm{GHz}$ )

- HP 8753ES S-Parameter Network Analyzer (30 kHz - 6 GHz) (Ref Only)

- Sunol Sciences FS-121 positioner with mast or turntable

- PC with LabVIEW and Matlab

- Wooden receiver antenna fixture

- Antenna Under Test (AUT)

- Receiver antenna

- Two 16ft SMA male to SMA male coaxial cables

- N male to SMA female adaptors

- $6 \mathrm{ft}$ RS-232 to USB cable

- $3 f t$ GPIB to USB cable

- VNA calibration kit

- Hardware and tools for mounting antennas

- Masking tape

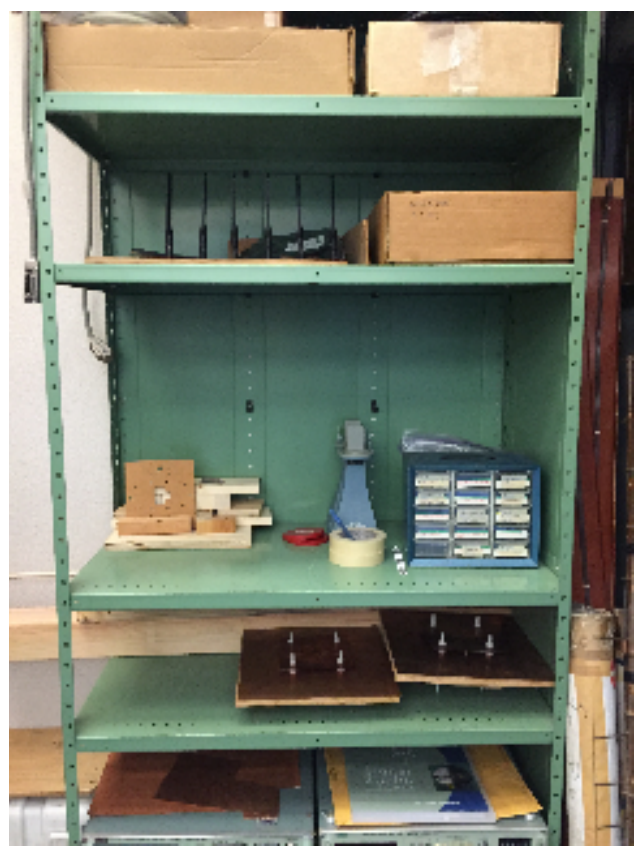

Figure 4 Parts Shelf

- Various wooden blocks

- Bubble level 


\section{Getting Started}

I. Mount the receiver antenna

A. Bolt the antenna by its adaptor to the wooden flange

1. Use washers to protect the surfaces

2. Nuts should only be hand tight

B. Connect the port 2 SMA cable to the waveguide adaptor

1. Use an $\mathrm{N}$ male to SMA female adaptor as needed

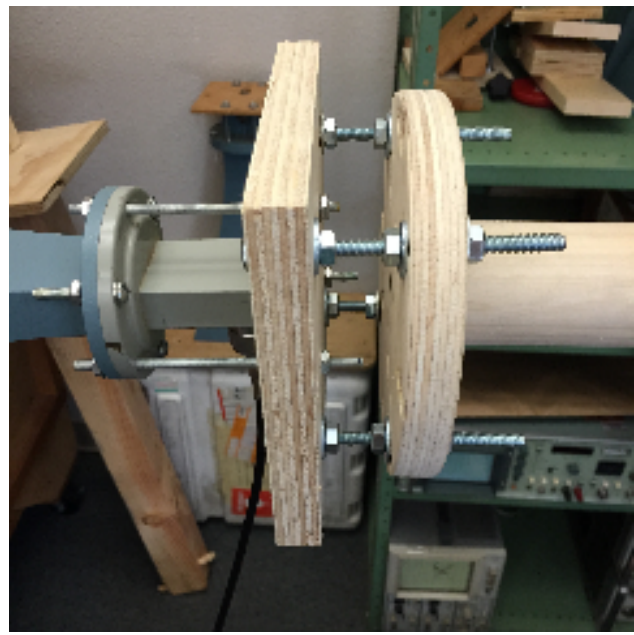

Figure 5 Antenna Adaptor

2. Do not over tighten

C. Move the fixture into position

1. Position the antenna with its aperture to the chamber window cutout

a) For large antennas, remove the foam window with the rectangular cutout

b) If positioning the antenna inside the chamber, be careful of the SMA cable

2. Align the antenna with the length of the chamber

3. Use the bubble level to verify the polarization

II. Mount the AUT

A. Remove the window at end ' $\mathrm{B}$ ' of the chamber

1. Do not put it on the floor

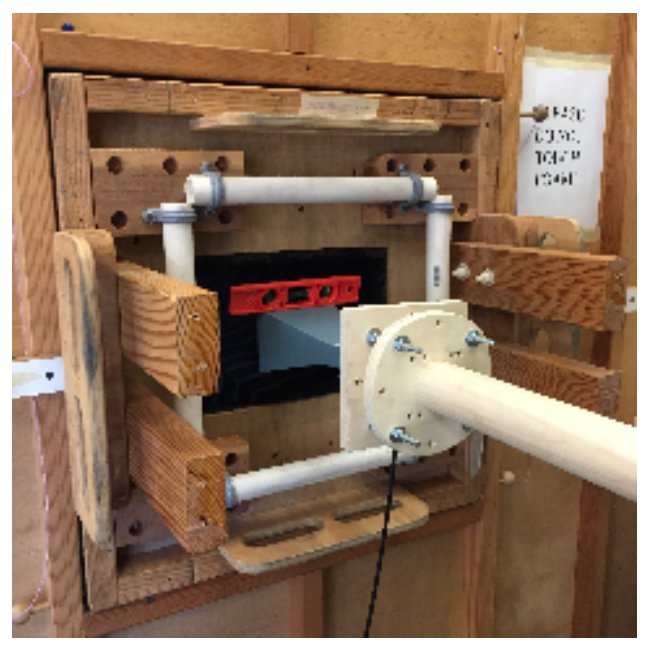

Figure 6 Test Antenna Setup 
B. Remove the clear acrylic mount from the AUT positioner fixture

1. It is safe to gently rotate the positioner manually

C. Bolt the antenna by its adaptor to the acrylic flange

1. Use washers to protect the surfaces

2. For small, lightweight antennas, use masking tape instead of bolts

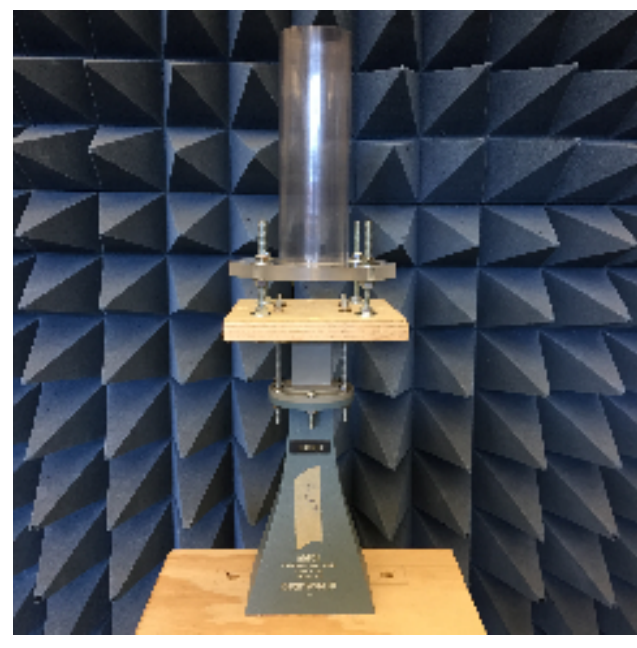

Figure 7 AUT Mount

D. Replace the acrylic mount with AUT in the positioner fixture

1. Be careful not to scrape the foam

E. Connect the port 1 SMA cable to the AUT

1. The SMA cable should feed up through the middle and out the side of the clear acrylic mast, then through one of the smaller holes in the horizontal arm of the black plastic fixture to the AUT.

a) Do not feed the SMA cable through the clear acrylic mount, as it will interfere with

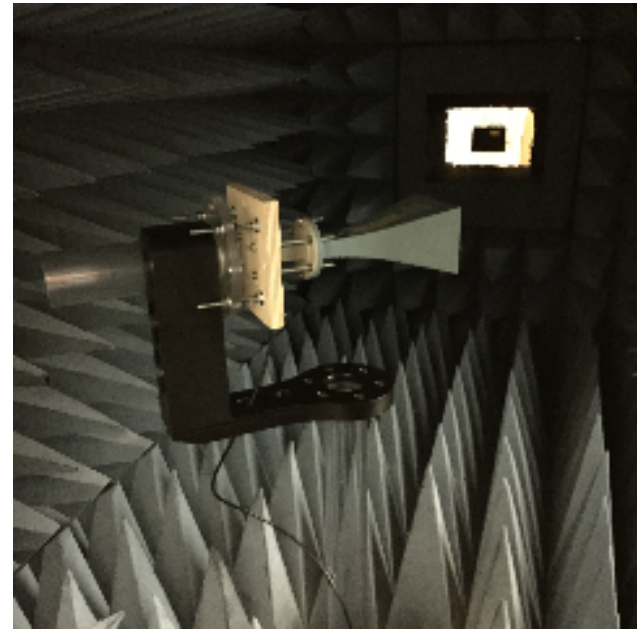

Figure 8 AUT in Chamber the foam during rotation.

2. Use an $\mathrm{N}$ to SMA female adaptor as needed

3. Do not over tighten

F. Point the AUT in the general direction of the receive antenna

1. Use the bubble level to verify the polarization 
III. Connect the VNA and Positioner to the PC and power them on

A. The positioner power is controlled by a surge protector on the desk

B. Port 1 should be connected to the AUT

C. Port 2 should be connected to the receive antenna

D. The VNA uses a GPIB to USB cable

E. The Positioner uses an RS-232 to USB cable

F. Wait for the VNA to complete its self-test

G. If the VNA is already on, reset it to the default configuration

IV. Start the program

A. Download and unzip the Quick Start folder to the desktop

1. Keep AMS.exe and the Matlab Scripts folder in the same directory

B. Run AMS.exe

1. A Matlab command window will open. Do not close it.

C. Click the run arrow in the toolbar and wait for the status window to display Ready

1. The program will now detect and initialize the VNA and positioner

2. The program will also automatically link to the Matlab Scripts folder

D. Select a folder to store your measurements using the Results Folder control on the left-hand column.

If any errors occur after executing the program, check that the VNA and positioner are connected to the computer and powered on, then click the run arrow. If the problem persists, close AMS.exe, reopen it, and try again. 


\section{Calibration}

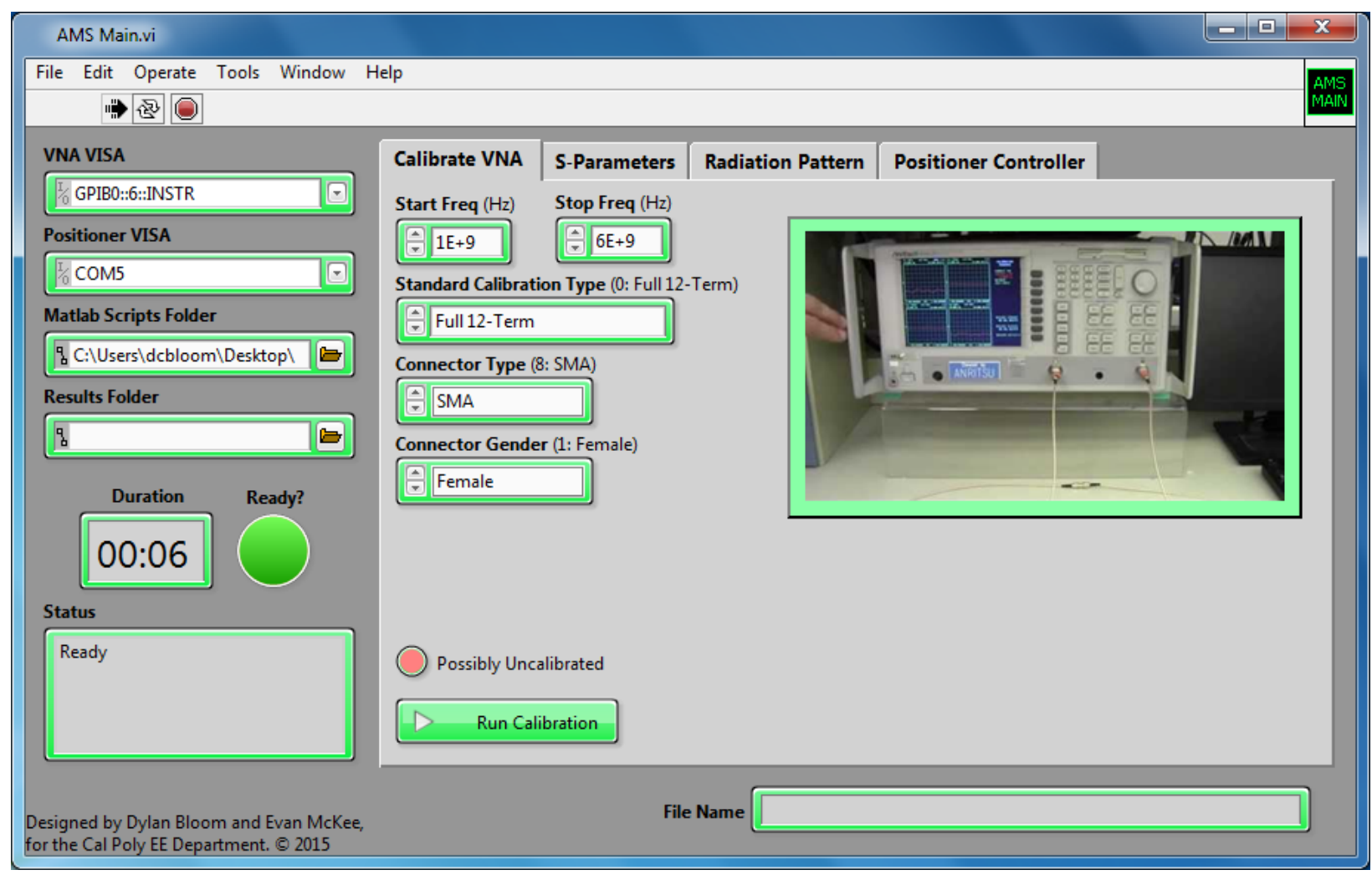

Figure 10 LabVIEW Front Panel: Calibration

There are two calibration types. Perform a Reflection Only calibration (faster) to just measure S11. Perform a Full 12-Term calibration to measure S11, S21, and radiation pattern. In the interest of time over accuracy, the user can skip calibration if only measuring S21 or radiation pattern, but should then normalize the data to zero. A UI button to normalize data is on the Radiation Pattern tab. Also, note the following:

- The system defaults to 1601 points.

- $\quad$ The user must manually calibrate the HP network analyzers.

- When measuring calibration standards, tape the ends of the SMA cables to somewhere convenient to make attaching the standards easier. Do not use tape on the foam. 


\section{Reflection Only Calibration}

I. Set calibration parameters
A. Set the Start Frequency and Stop Frequency
B. Set the Standard Calibration Type to Reflection Only Forward
C. Select the Connector Type
D. Select the Connector Gender

II. Perform the calibration

A. Click the Run Calibration button

1. LabVIEW will now program the VNA

2. Wait for a prompt that says "Measure Device"

B. Measure the calibration standards

1. Read the VNA screen to see what device to connect

2. For each calibration standard, connect it to the cable and click OK in the Measure Device prompt, then wait for the measurement to finish.

3. The VNA will beep after completing each device

4. Table 1 shows the calibration sequence

Table 1 Reflection Only Calibration Sequence

\begin{tabular}{|r|l|}
\hline Step & Port 1 \\
\hline $\mathbf{1}$ & Open \\
\hline 2 & Short \\
\hline $\mathbf{3}$ & Broadband Load \\
\hline
\end{tabular}

III. Reconnect the cable to the antenna to be measured 


\section{Full 12-Term Calibration}

I. Position the AUT at boresight

A. Go to the Positioner Controller tab

B. If the AUT does not have a distinct main beam, it must be positioned manually

1. Gently position the AUT by hand

2. Alternatively, use the quick step buttons

a) Use New Angle, CW Step, and CCW Step. to locate the AUT

b) Do not go beyond $\pm 360^{\circ}$, as this could damage the SMA cable

c) Click Set This Position to Zero

3. Skip to step II

C. If the AUT does have a distinct main beam, the program can position it automatically

1. Set Freq above the Auto Locate Boresight Position button to your frequency of interest

2. Click the Auto Locate Boresight Position button

a) The Precision controls the increment the program will use when auto locating

b) This can be done with window 'B' open.

3. Wait for the Current Angle indicator to display 0

D. Verify the AUT is at boresight

1. Look through the window on side 'A' of the chamber with a flashlight

2. If the AUT is off boresight, use the CW Step and CCW Step buttons to manually align it, and click Auto Locate Boresight Position again. 
3. If that does not work, use the quick step buttons to position the AUT manually. See step B.

II. Set calibration parameters
A. Set the Start Frequency and Stop Frequency
B. Set the Standard Calibration Type to Full 12-Term
C. Select the Connector Type
D. Select the Connector Gender

III. Perform the calibration
A. Click the Run Calibration button

1. LabVIEW will now program the VNA

2. Wait for a prompt that says "Measure Device"

B. Measure the calibration standards

1. Read the VNA screen to see what devices to connect

2. For each calibration standard, connect it to the cable and click OK in the Measure Device prompt, then wait for the measurement to finish.

3. The VNA will beep after completing each device

4. Table 2 shows the calibration sequence

Table 2 Full 12-Term Calibration Sequence

\begin{tabular}{|r|l|l|}
\hline Step & Port 1 & Port 2 \\
\hline $\mathbf{1}$ & Broadband Load & Broadband Load \\
$\mathbf{2}$ & Open & Short \\
$\mathbf{3}$ & Short & Open \\
\hline $\mathbf{4}$ & Through Line & Through Line \\
\hline
\end{tabular}




\section{Taking Measurements}

After taking measurements, the program automatically builds a descriptive file name and saves the data to a tab delimited text file with headers in the Results Folder. The file name is displayed below the plots. Note that if the same measurement is performed twice, previous data will be overwritten.

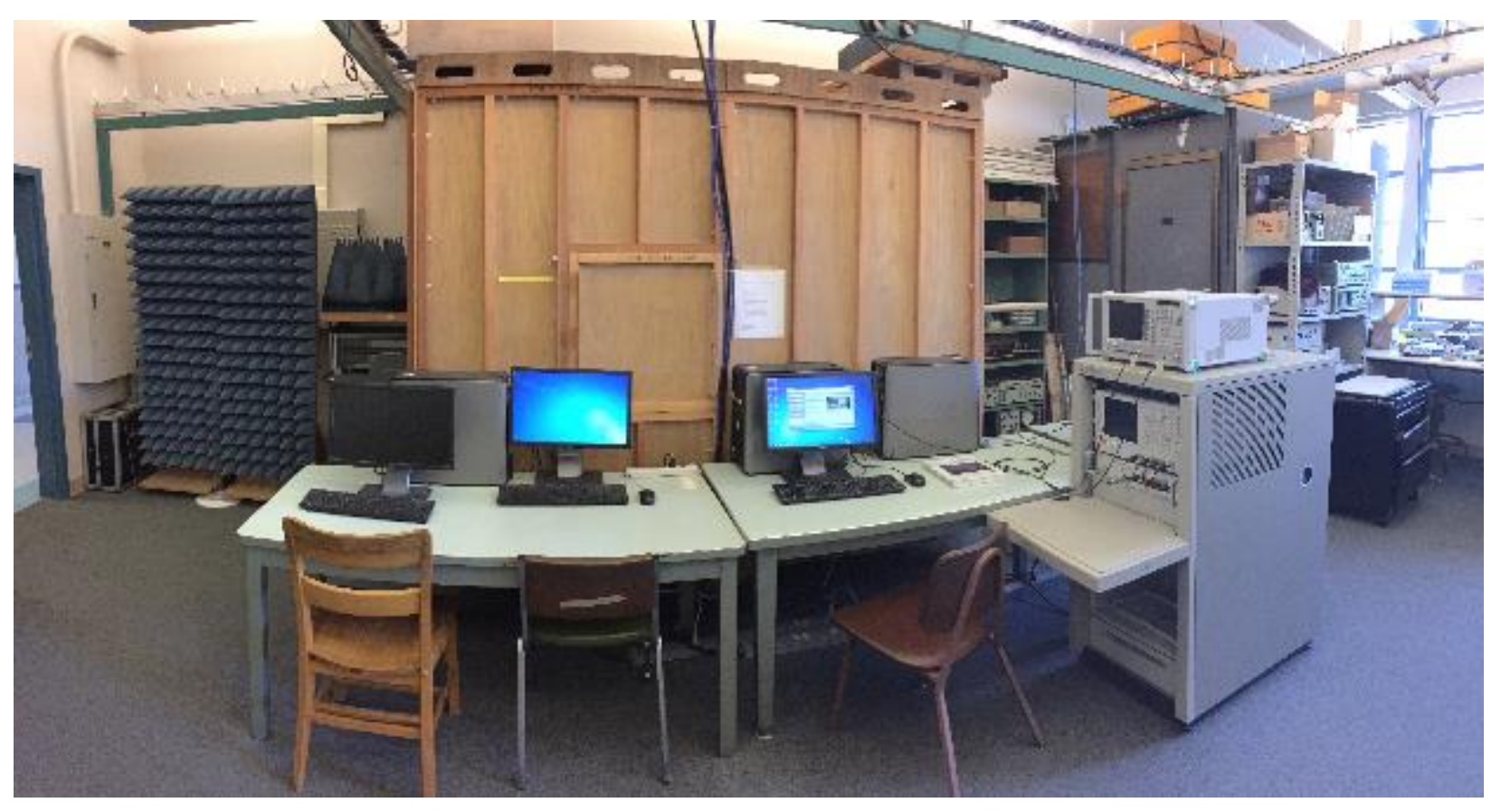

Figure 11 The Microwave Lab Anechoic Chamber 


\section{S-Parameters}

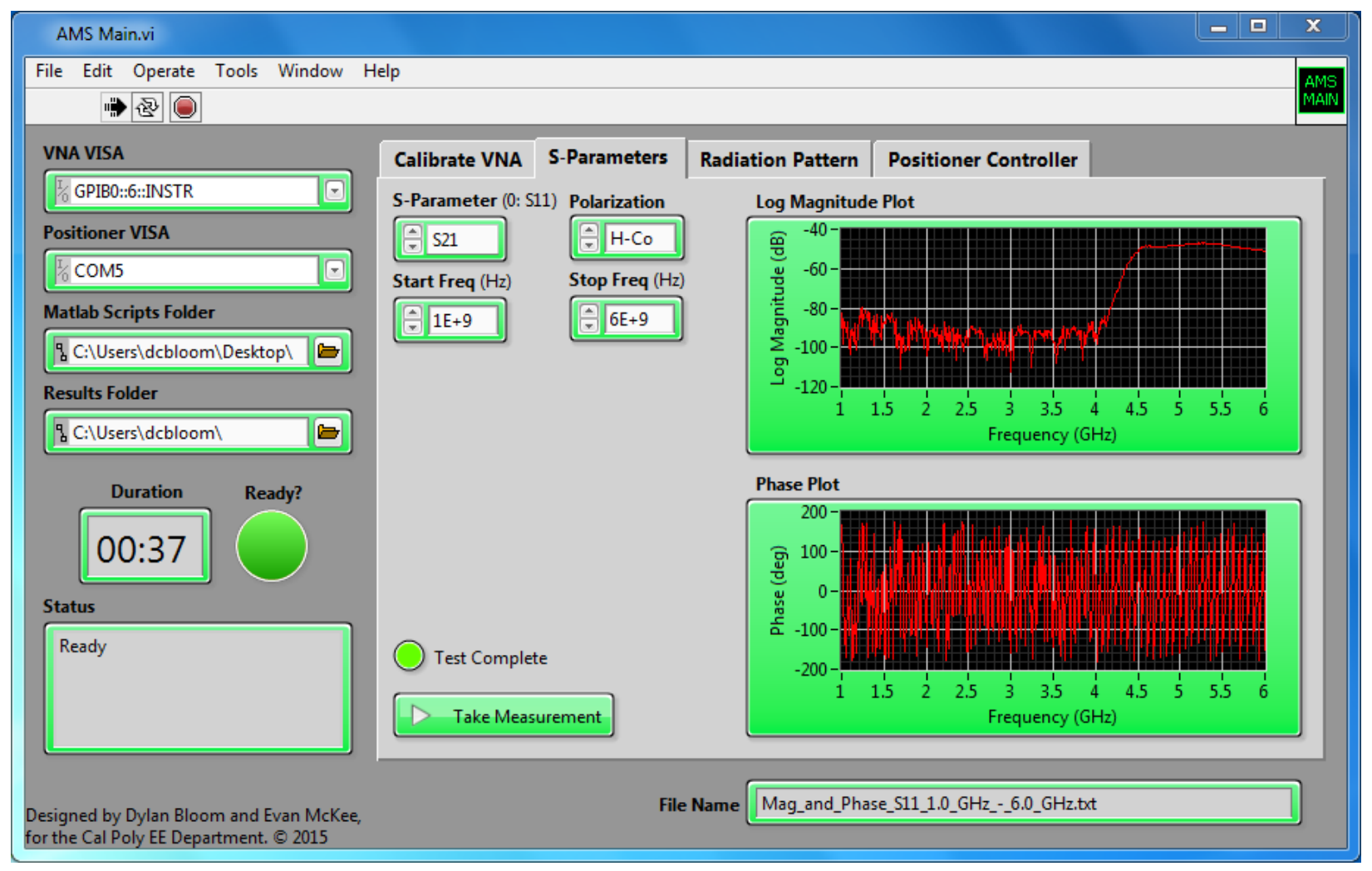

Figure 12 LabVIEW Front Panel: Uncalibrated S21 Measurement

IV. Close the chamber windows

V. Go to the S-Parameters tab

VI. Select desired S-Parameter

VII. If measuring S21 or S12, enter the Polarization configuration

VIII.Enter the Start Frequency and Stop Frequency

IX. Click the Take Measurement button

A. After a few seconds, Log Magnitude and Phase data will display on the plots. 


\section{Radiation Pattern}

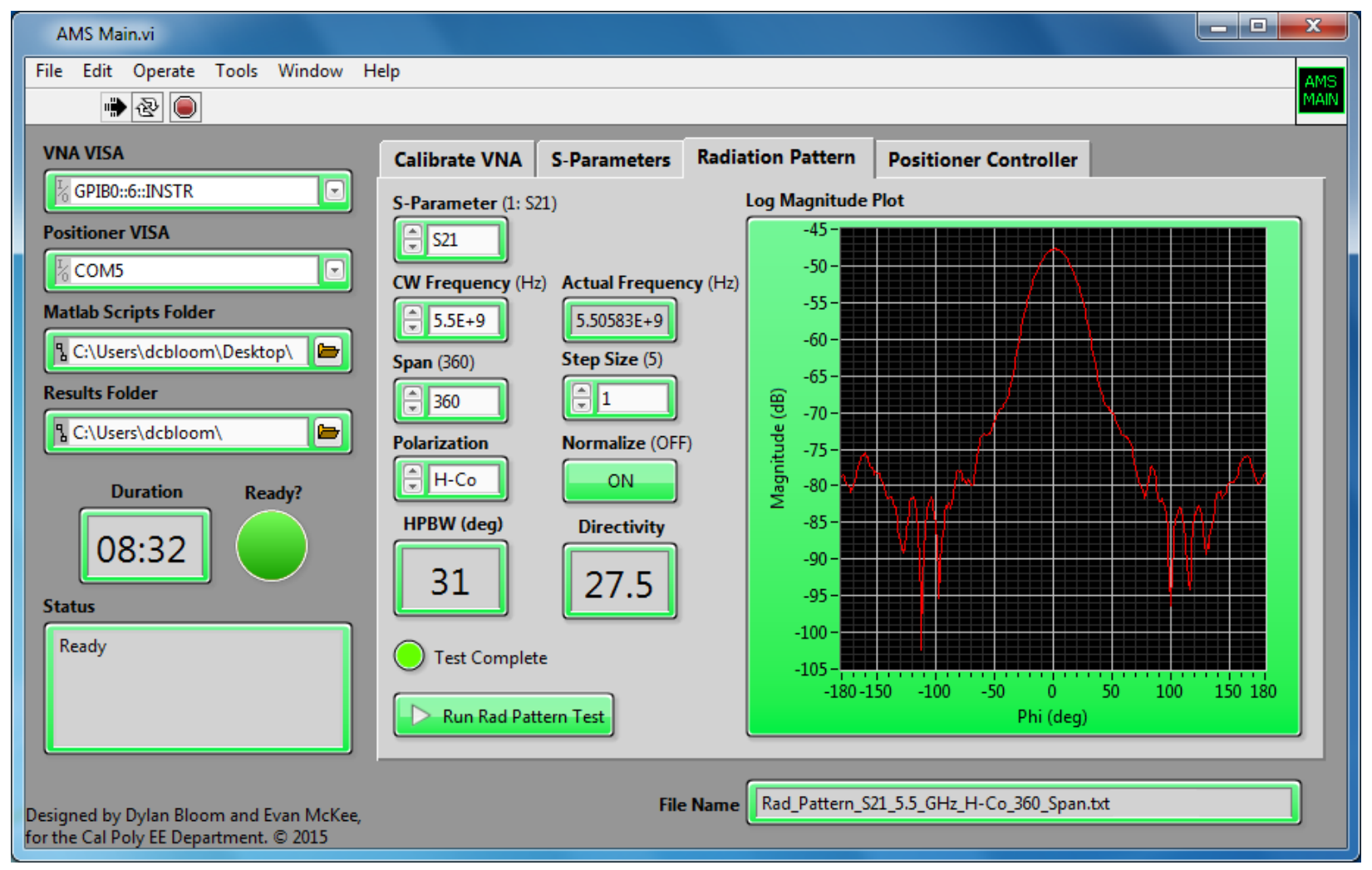

Figure 13 LabVIEW Front Panel: Uncalibrated Radiation Pattern Measurement

I. Close the chamber windows

II. Go to the Radiation Pattern tab

III. Select the desired S-Parameter

\section{Select a CW Frequency}

\section{Set the Span and Step Size}

A. The program uses Span to determine the start and stop angles, but $0^{\circ}$ is always the center angle.

VI. Enter the Polarization configuration

VII. If you skipped the calibration step, switch Normalize to ON 
A. Normalize simply tells Matlab to plot the polar radiation pattern such that the maximum log magnitude is scaled to $0 \mathrm{~dB}$. This scaling is only temporary and does not affect the data that gets saved.

\section{VIII.Click Run Rad Pattern Test}

A. The positioner will first rotate the AUT to $-S p a n / 2$, then step through to + Span/2, plotting each data point as it is measured.

B. To cancel a sweep, click the Cancel Sweep button

C. After the last measurement

1. The positioner returns to $0^{\circ}$

2. The tabular data gets saved to a text file

3. Matlab plots the logarithmic polar radiation pattern

a) Note you must manually save the figure as a .png

4. Matlab calculates the HPBW, and estimates the Directivity

a) Note these values are not saved

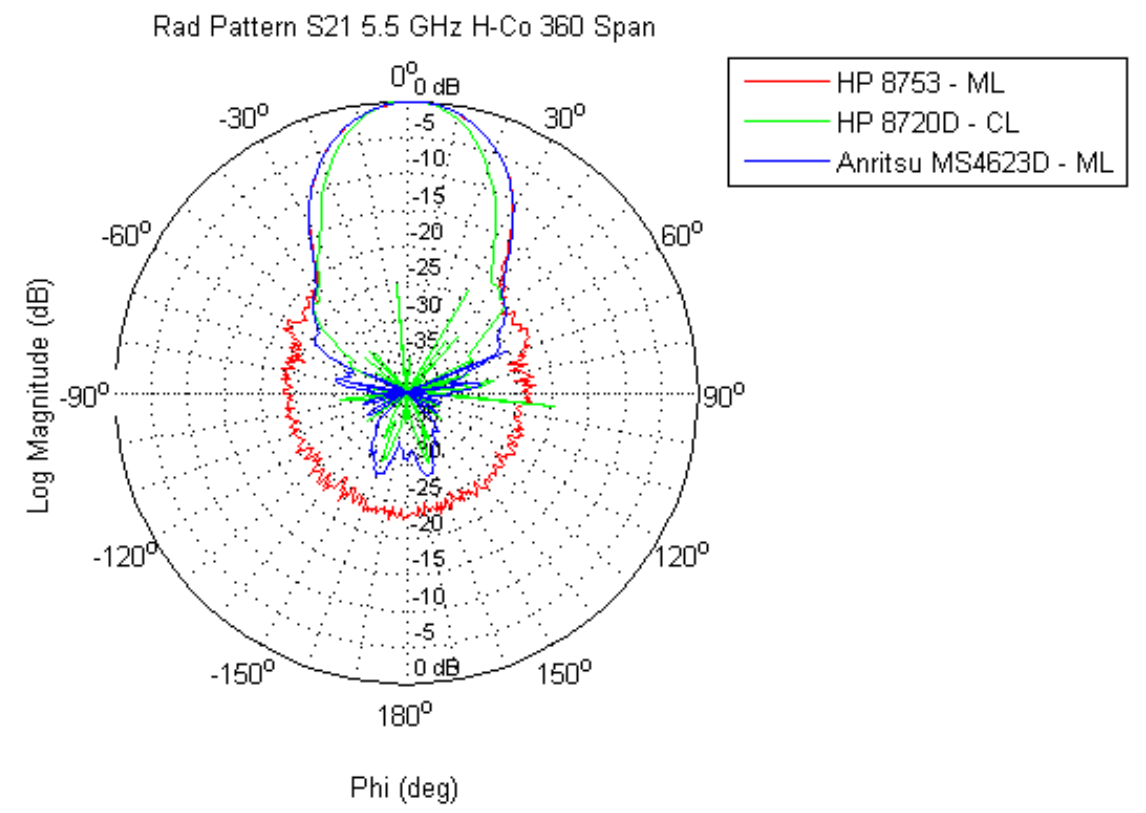

Figure 14 Matlab Pattern Plot Comparison of the Narda 643 Antenna $(\mathrm{ML}=$ Microwave Lab) $(\mathrm{CL}=$ Hangar Lab) 


\section{APPENDIX F: RadPatternPlot.m}

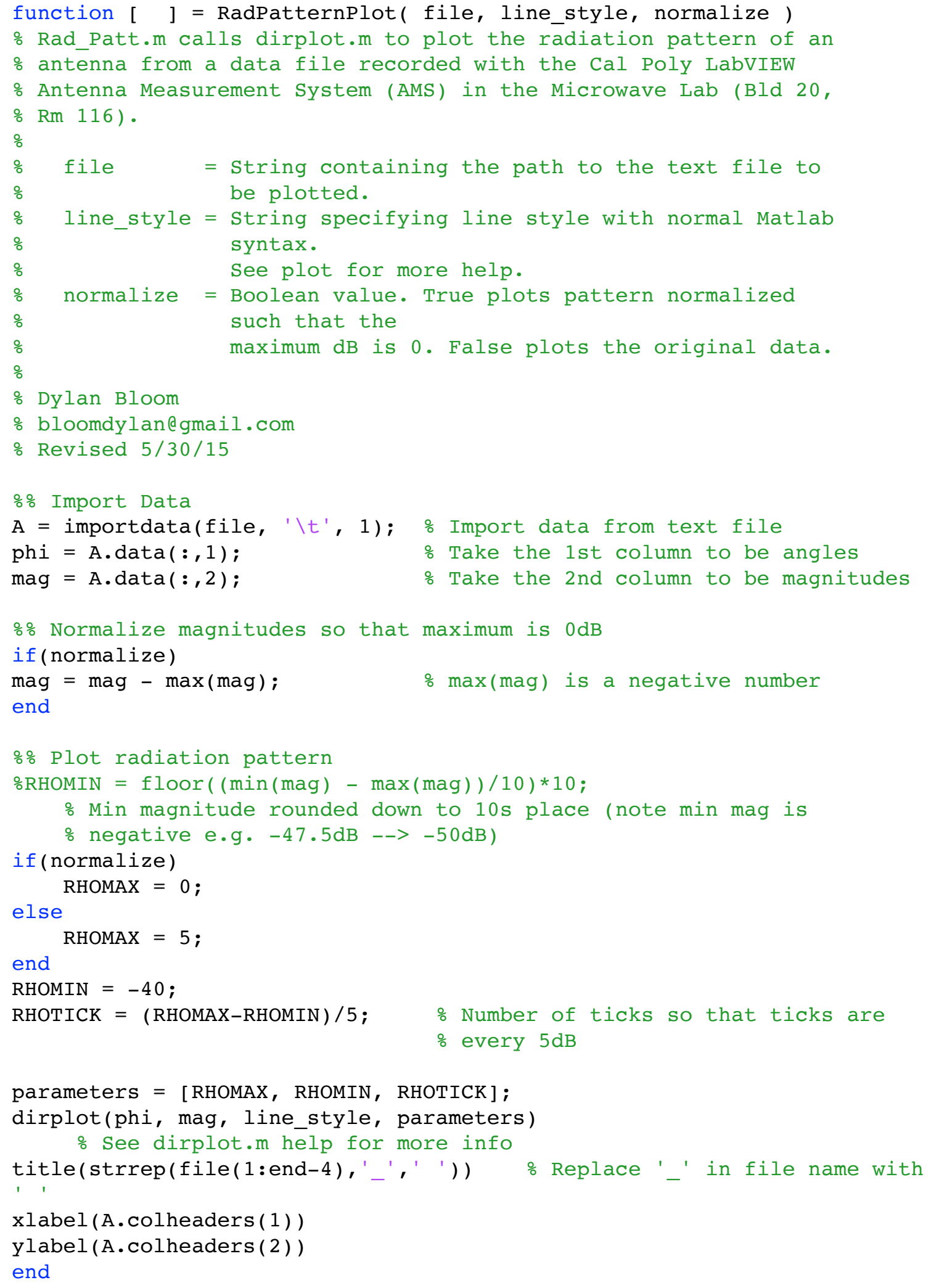




\section{APPENDIX G: Directivity.m}

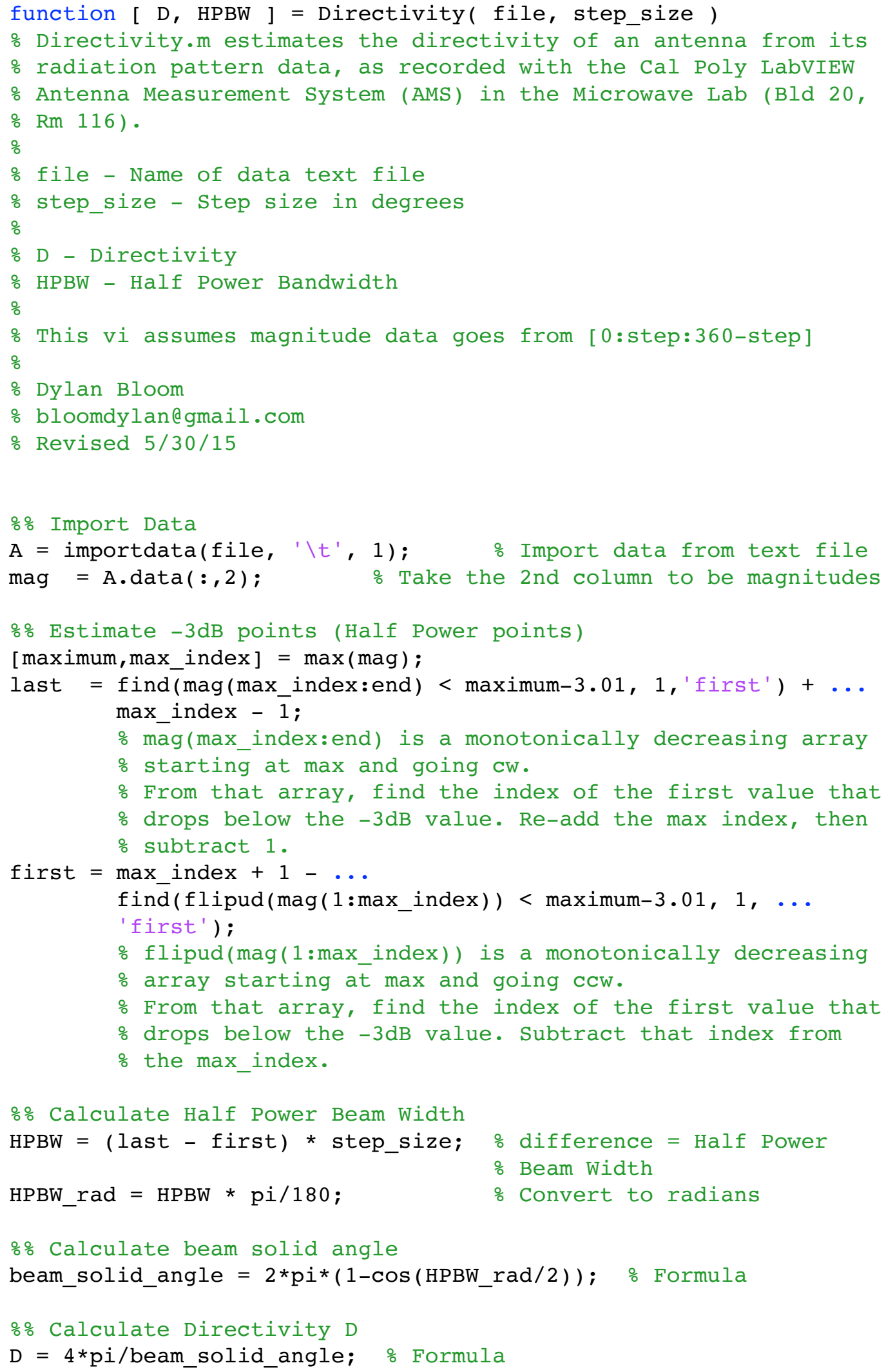




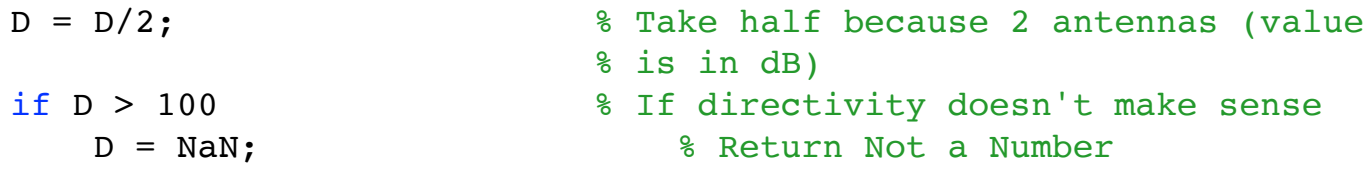


Read section 9.4.3 The Pyramidal Horn Antenna in Antenna Theory and Design 3rd ed., and use the design equations to design a $2.4 \mathrm{GHz}$ pyramidal horn antenna. Use the following figure from the book for reference.

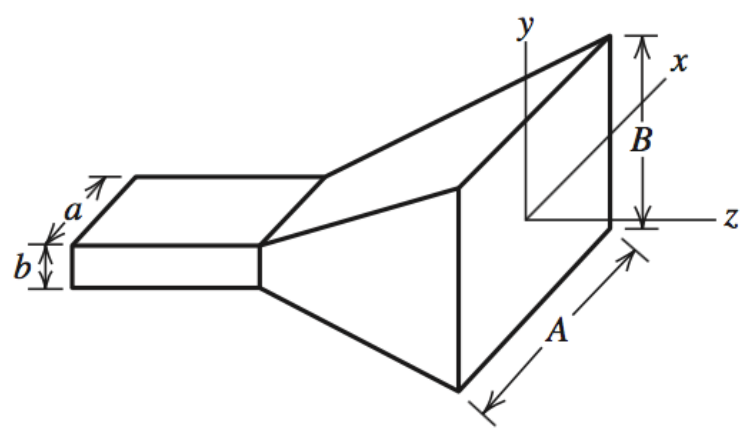

(a) Overall geometry.

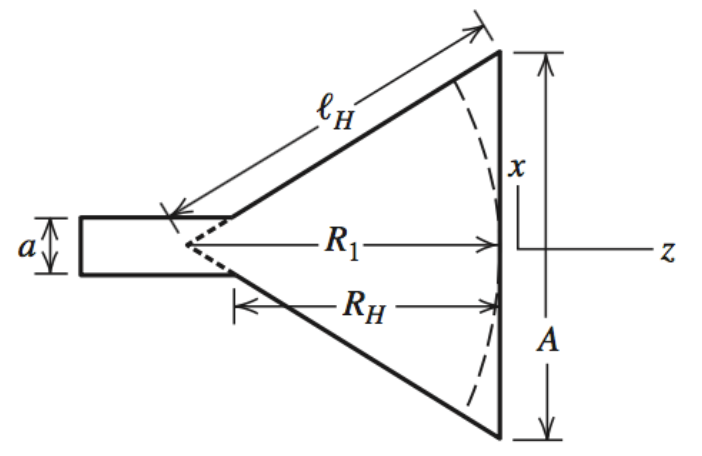

(b) Cross section through the $x z$-plane ( $H$-plane).

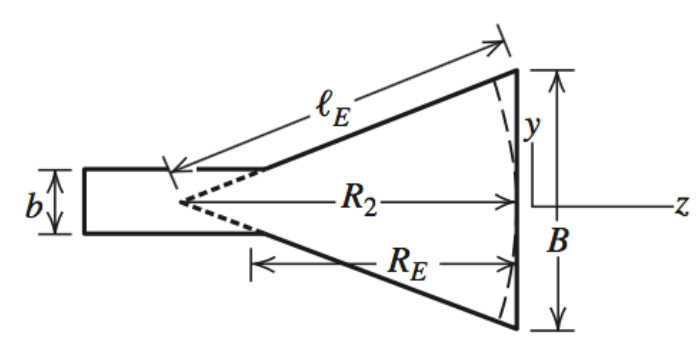

(c) Cross section through the $y z$-plane ( $E$-plane).

\section{Getting Started}

I. Launch ANSYS HFSS

II. Verify Tools Settings

A. Select the menu item Tools > Options > HFSS Options...

B. HFSS Options Window:

1. Duplicate boundaries/mesh operations with geometry: Checked

2. Click OK.

C. Select the menu item Tools > Options > Modeler Options...

D. Modeler Options Window:

1. Click the Drawing tab

a) Edit properties of new primitives: Checked

2. Click OK. 
III. Open a New Design

A. Select the menu item Project > Insert HFSS Design

IV. Set Solution Type

A. Select the menu item HFSS > Solution Type...

B. Solution Type: "Project Name - Design Name" Window:

1. Choose Driven Modal Network Analysis

2. Click OK

\section{Creating the 3D Model}

I. Set Model Units

A. Select the menu item Modeler > Units...

1. Set Model Units Window:

a) Select units: $\mathbf{c m}$

b) Click OK

II. Set Default Material

A. From the drawing toolbar drop down menu, choose vacuum

III. Set Drawing Plane

A. From the drawing toolbar drop down menu, change $X Y$ to $Y Z$

IV. Create the Rectangular Waveguide

A. Select the menu item Draw $>$ Box

B. Draw an arbitrary box and a properties window will appear

C. Properties Window:

1. Position: $\mathbf{0}$, -waveguide_width/2, -waveguide_height/2

2. Add Variable Window:

a) Enter design values for each parameter followed by "cm"

b) waveguide_width $=\boldsymbol{a}$ from book

c) waveguide_height $=\boldsymbol{b}$ from book

3. XSize: waveguide_length

4. Add Variable Window:

a) waveguide_length $=7 \mathbf{c m}$

5. YSize: waveguide_width

6. ZSize: waveguide_height

7. Click OK

D. Set the Object Name

1. From the Properties toolbox on the left, set the name to Waveguide

E. Fit the View

1. Press CTRL+D

F. From the Properties toolbox click the Transparent Value

1. Set Transparency Dialog:

a) Set Transparency to $\mathbf{0 . 5}$

b) Click OK

V. Create the Horn Base

A. Select the menu item Draw $>$ Rectangle 
B. Draw an arbitrary rectangle

C. Properties Window:

1. Position: waveguide_length, -waveguide_width/2, -waveguide_height/2

2. YSize: waveguide_width

3. ZSize: waveguide_height

4. Click OK

D. Set the Object Name

1. From the Properties toolbox on the left, set the name to Horn_Base

VI. Create the Horn Top
A. Select the menu item Draw > Rectangle
B. Draw an arbitrary rectangle
C. Properties Window:

1. Position: horn_length+waveguide_length, -aperture_width/2, aperture_height/2

2. Add Variable Window:

a) Enter design values for each parameter followed by "cm"

b) horn_length $=\boldsymbol{R}_{\boldsymbol{P}}$ from book

c) aperture_width $=\boldsymbol{A}$ from book

d) aperture_height $=\boldsymbol{B}$ from book

3. YSize: aperture_width

4. ZSize: aperture_height

5. Click OK

D. Set the Object Name

1. From the Properties toolbox on the left, set the name to Horn_Top

E. Fit the View

1. Press CTRL+D

VII. Connect the Objects

A. Select the Horn_Base and Horn_Top from the Modeler design tree.

B. Select the menu item Modeler $>$ Surface $>$ Connect

C. Name the object Horn

VIII.Complete the Antenna
A. Select all objects
1. Press CTRL+A
B. Select the menu option Modeler $>$ Boolean $>$ Unite

IX. Create an Air Box around the Horn Antenna
A. Select the menu item Draw $>$ Box
B. Draw an arbitrary box and a properties window will appear
C. Properties Window:

1. Position: 0 , -aperture_width* ${ }^{\star} 1.1 / 2$, -aperture_height ${ }^{\star} 1.1 / 2$

2. XSize: (horn_length+waveguide_length) ${ }^{*} 1.05$

3. YSize: aperture_width*1.1

4. ZSize: aperture_height ${ }^{\star} 1.1$

5. Click OK

D. Set the Object Name

1. From the Properties toolbox on the left, set the name to Air 
E. Fit the View

1. Press CTRL+D

F. From the Properties toolbox click the Transparent Value

1. Set Transparency Dialog:

a) Set Transparency to $\mathbf{0 . 9}$

b) Click OK

\section{Assigning Boundaries and Excitations}

I. Create the Wave Port

A. Select the menu item Edit $>$ Select $>$ Faces

B. Select the menu item Edit > Select > By Name...

C. Select Face Dialog:

1. Choose the back face of the Waveguide

2. Click OK

D. Select the menu item HFSS > Excitations > Assign > Wave Port...

E. Wave Port Window:
1. Name: 1
2. Click Next
3. Click Next
4. Click Finish

II. Assign a Radiation Boundary

A. Select the menu item Edit $>$ Select $>$ Objects

B. Select the menu item Edit $>$ Select $>$ By Name...

C. Select Object Dialog:

1. Choose the Air

2. Click OK

D. Select the menu item HFSS > Boundaries > Assign > Radiation...

E. Radiation Boundary Window:

1. Name: Rad1

2. Click OK

III. Assign the Antenna a Perfect Electric Field Boundary

A. Select the menu item Edit $>$ Select $>$ Faces

B. Select the menu item Edit > Select > By Name...

C. Select Face Dialog:

1. Select all faces of the Horn except the aperture

2. Click OK

D. Select the menu item HFSS > Boundaries > Assign > Perfect E...

E. Perfect E Boundary Window:

1. Name: Horn_Walls

2. Click OK

\section{Setting up the Analysis}

I. Create an Analysis Setup

A. Select the menu item HFSS > Analysis Setup > Add Solution Setup...

B. Driven Solution Setup Window: 
1. Name: Setup1

2. Solution Frequency: $2.4 \mathrm{GHz}$

3. Maximum Number of Passes: 20

4. Maximum Delta S: $\mathbf{0 . 0 2}$

5. Click OK

C. Select the menu item HFSS > Analysis Setup > Add Sweep...

D. Select Setup1

E. Edit Frequency Sweep Window:

1. Sweep Name: Sweep

2. Sweep Type: Fast

3. Type: LinearCount

4. Start: $1 \mathrm{GHz}$

5. Stop: $6 \mathbf{G H z}$

6. Count: $\mathbf{1 6 0 1}$

7. Click OK

II. Validate the Model

A. Select the menu item HFSS > Validation Check...

B. There should only one warning about the Perf E boundary overlapping the Radiation Boundary. This can be ignored.

\section{Analyzing and Creating Reports}

I. Analyze the Model

A. Select the menu item HFSS > Analyze AII

II. Create an S11 Plot

A. Select the menu item HFSS > Results > Create Modal Solution Data Report > Rectangular Plot

B. Report Window:

1. Click New Report

2. Click Close

III. Create a Far Field Setup

A. Select the menu item HFSS > Radiation > Insert Far Field Setup > Infinite Sphere...

B. Far Field Radiation Sphere Setup Window:

1. Name: Infinite Sphere1

2. Phi Start: $\mathbf{- 1 8 0}$

3. Phi Stop: $\mathbf{1 8 0}$

4. Phi Step Size: 1

5. Theta Start: 0

6. Theta Stop: $\mathbf{1 8 0}$

7. Theta Step Size: 10

8. Click OK

IV. Create a Far Field 3D Polar Plot

A. Select the menu item HFSS $>$ Results $>$ Create Far Fields Report $>3$ D Polar Plot 
B. Report Window:

1. Mag: $\mathbf{d B}$ (rETotal)

2. Click New Report

3. Click Close

V. Overlay the 3D radiation pattern on the model

A. Double click on the design in the project tree

B. Select the menu item HFSS > Fields > Plot Fields > Radiation Field...

C. Overlay Radiation Field Window:
1. Visible: Checked
2. Transparency: $\mathbf{0 . 5}$
3. Scale: $\mathbf{1 . 0}$
4. Click Apply
5. Click Close
6. Press CTRL+D

VI. Create a Far Field Rectangular Plot
A. Select the menu item HFSS > Results > Create Far Fields Report > Rectangular Plot

B. Report Window:
1. Primary Sweep: Phi
2. Mag: $\mathbf{d B}$ (rETotal)
3. Click New Report
4. Click Close

\section{Exporting Data from HFSS}

I. Export Rectangular Field Plot Data
A. Select the Rectangular Field Plot under Results in the project tree
B. Select the menu item Report2D > Export...
C. Export Report Dialog:
1. Click Browse
2. File Name: HFSS Rectangular Field Plot

II. Modify the file for Matlab
A. Open the .txt file in Excel
B. Delete all columns except for Phi [deg] and Theta='90deg'
C. Delete rows 1-5 and 7
D. Press $\mathbf{C T R L}+\mathbf{S}$

III. Export Rectangular S11 Plot Data
A. Repeat steps I and II above (except II B) for the Rectangular S11 Plot
B. File Name: HFSS S11 Plot 